\title{
The role of the lung in shaping CNS autoimmunity
}

\author{
Dissertation \\ for the award of the degree \\ "Doctor rerum naturalium" \\ of the Georg-August-Universität Göttingen
}

within the doctoral program "Molecular Biology of Cells"

of the Georg-August University School of Science (GAUSS)

submitted by

Leon Hosang

from Uelzen

Göttingen, 2019 


\section{Thesis Committee}

Prof. Dr. Alexander Flügel

Institute for Neuroimmunology and Multiple Sclerosis Research University Medical Center Göttingen (UMG)

Prof. Dr. Holger Reichardt

Institute for Cellular and Molecular Immunology

University Medical Center Göttingen (UMG)

Prof. Dr. Wolfgang Brück

Institute of Neuropathology

University Medical Center Göttingen (UMG)

\section{Members of the Examination Board}

Referee: Prof. Dr. Alexander Flügel

Institute for Neuroimmunology and Multiple Sclerosis Research

University Medical Center Göttingen (UMG)

$2^{\text {nd }}$ Referee: Prof. Dr. Holger Reichardt

Institute for Cellular and Molecular Immunology

University Medical Center Göttingen (UMG)

\section{Further Members of the Examination Board}

Prof. Dr. Wolfgang Brück Institute of Neuropathology

University Medical Center Göttingen (UMG)

Prof. Dr. Jürgen Wienands

Institute for Cellular and Molecular Immunology

University Medical Center Göttingen (UMG)

Prof. Dr. Christine Stadelmann-Nessler

Institute of Neuropathology

University Medical Center Göttingen (UMG)

Prof. Dr. Martin S. Weber

Institute of Neuropathology

University Medical Center Göttingen (UMG) 


\section{Table of Contents}

\section{List of Figures}

List of Tables

List of Abbreviations

\section{Summary}

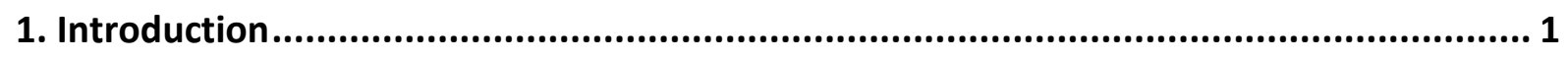

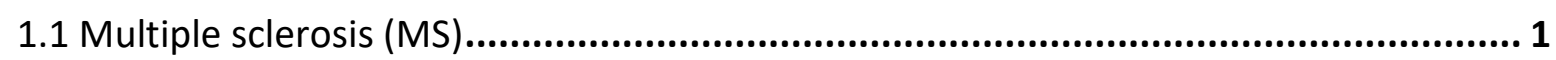

1.1.1 Clinical picture and epidemiology of MS ........................................................ 1

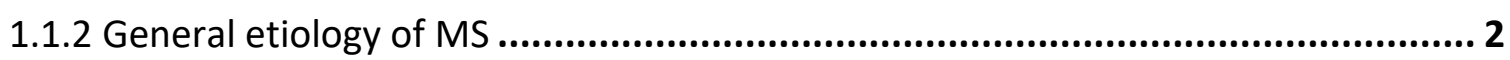

1.1.3 Lung-associated MS risk factors ...................................................................... 3

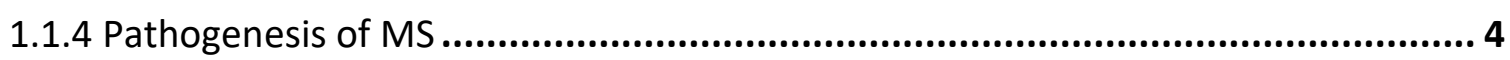

1.2 Experimental autoimmune encephalomyelitis (EAE) ........................................... 4

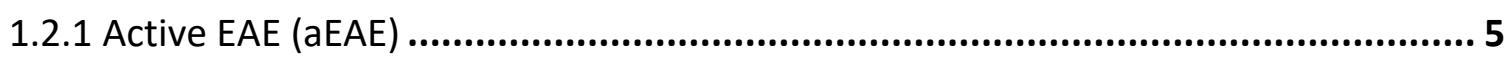

1.2.2 Passive transfer EAE (ptEAE) ................................................................. 5

1.2.3 The lung as a critical site of T cell reprogramming in EAE ................................. 6

1.3 Potential mechanistic links between lung and CNS autoimmunity............................ 8

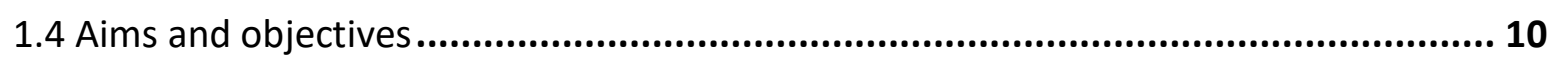

1.4.1 Establishment and characterization of an i.tr. induced EAE model ..................... 10

1.4.2 Effect of intrapulmonary antibiotic treatment on EAE .................................... 10

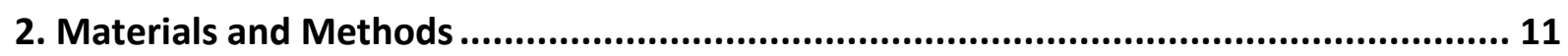

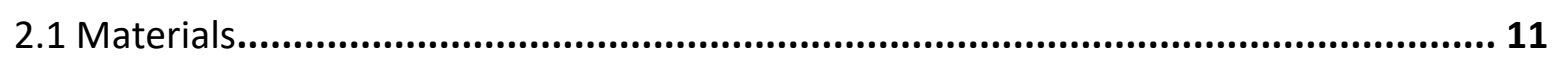

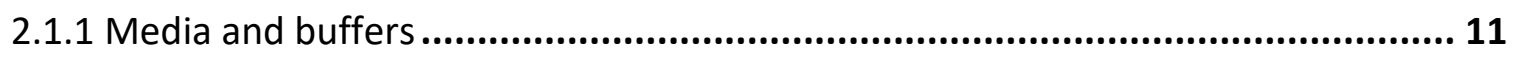

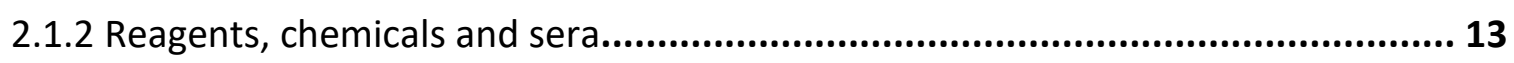

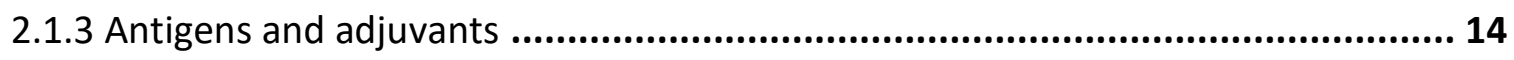

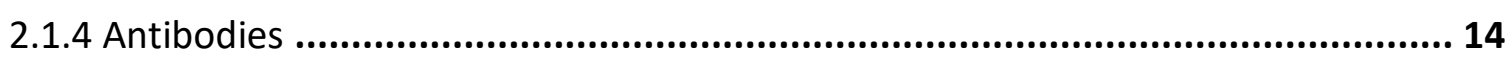

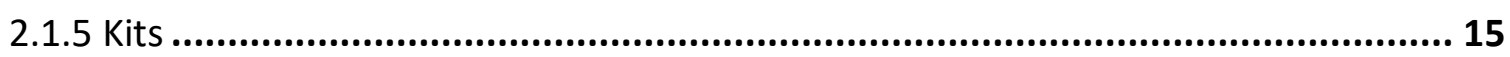

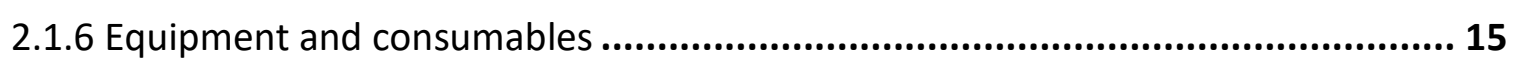

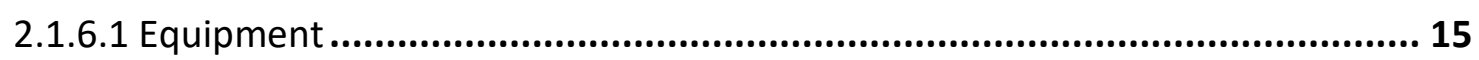

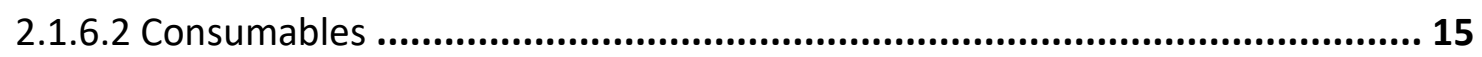

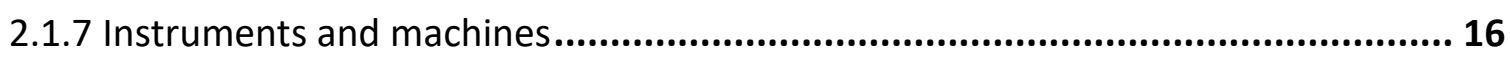

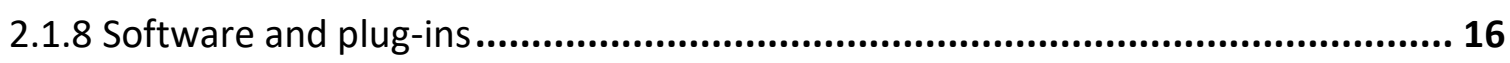

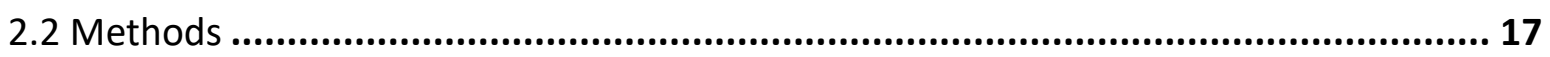

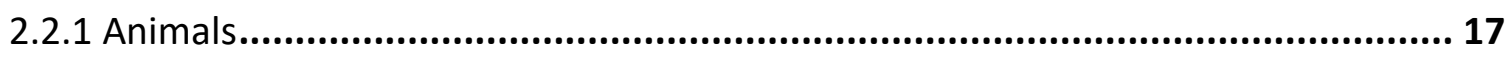

2.2.2 Generation and culture of primary $T$ cell lines.............................................. 17

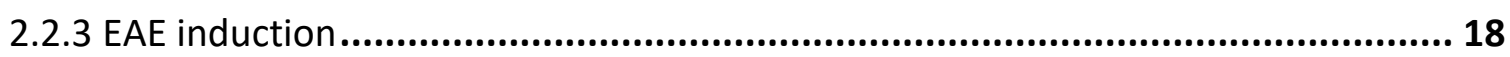




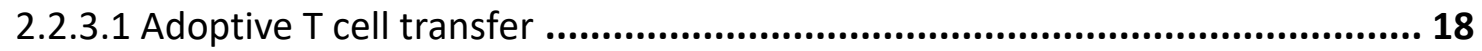

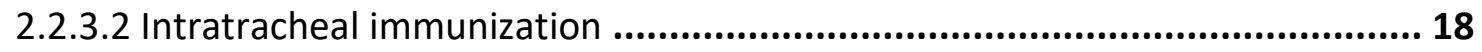

2.2.3.3 Subcutaneous immunization............................................................... 19

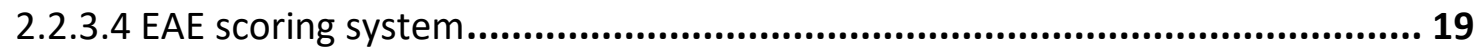

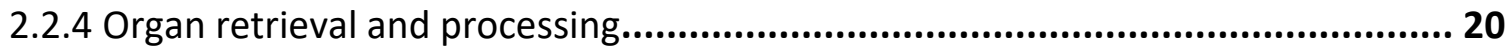

2.2.4.1 Preparation of single cell suspensions from different organs ........................ 20

2.2.4.2 Isolation of antigen-presenting cells from the lung to stimulate $T_{M B P-E M}$ cells. 21

2.2.4.3 Sorting of endothelial cells from spinal cord parenchyma and meninges....... 21

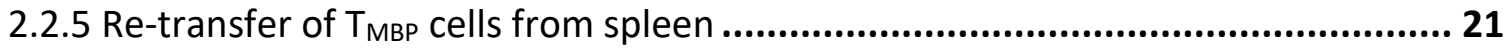

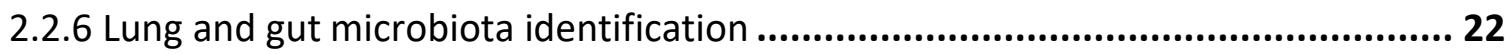

2.2.6.1 Intratracheal administration of neomycin............................................. 22

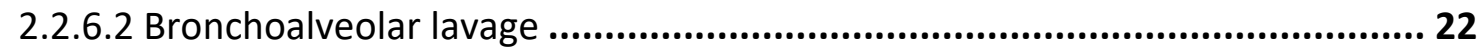

2.2.6.3 DNA isolation from bronchoalveolar lavage (BAL) fluid ............................ 22

2.2.6.4 DNA isolation from fecal samples............................................................. 23

2.2.6.5 BAL fluid and fecal sample DNA quantification........................................... 23

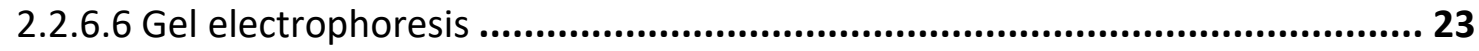

2.2.6.7 PCR amplification of bacterial amplicons ................................................... 23

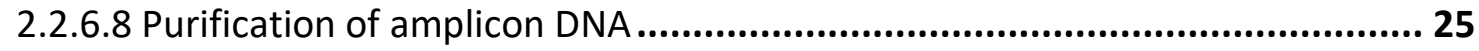

2.2.6.9 Bacterial amplicon sequencing, data processing and data analysis................ 25

2.2.7 $\mathrm{T}_{\text {MBP-EM }}$ cell activation and proliferation assay .................................................. 25

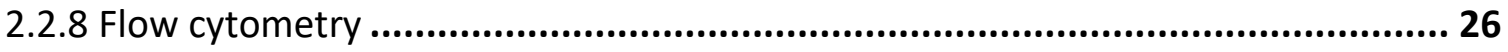

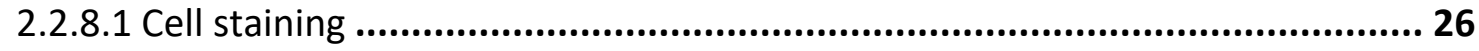

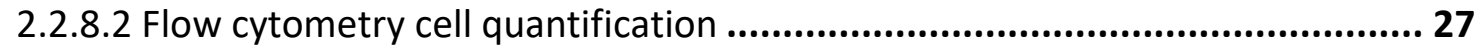

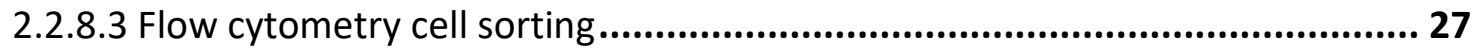

2.2.9 RNA isolation, cDNA synthesis and quantitative real-time PCR......................... 30

2.2.10 Next-Generation Sequencing of sorted T cells.................................................. 31

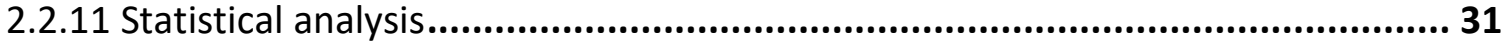

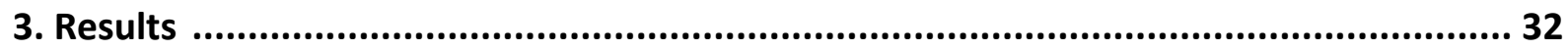

3.1. TMBP-EM cell activation and EAE induction via the lung ........................................... 32

3.1.1 Establishment and characterization of an i.tr. induced EAE model ..................... 32

3.1.1.1 Intratracheal immunization with low doses of MBP induces EAE .................. 32

3.1.1.2 Moderate CFA concentrations are sufficient to induce EAE via the lung ......... 33

3.1.1.3 Immunization with a low MBP concentration induces EAE in memory rats.... 34

3.1.1.4 Kinetic of $T_{M B P}$ cell distribution during i.tr. induced EAE.............................. 35

3.1.1.5 Kinetic of $T_{M B P}$ cell activation during i.tr. induced $E A E$............................... 39

3.1.1.6 Gene expression of $T_{M B P}$ cells is highly regulated during i.tr. induced $E A E$..... 41 
3.1.1.7 Antigen-presenting cells from the lung are capable of activating TMBP-EM cells 44

3.1.2 Examination of the antigen dose-dependent difference in EAE severity 46

3.1.2.1 Lung immunization with high antigen doses does not appear to locally trap immune cells

3.1.2.2 $\mathrm{T}_{\mathrm{MBP}-\mathrm{EM}}$ cells activated in the lung with high antigen concentrations do not appear to be anergic or impaired in their proliferation potential

3.1.2.3 The antigen dose-dependent difference in EAE severity is T cell-intrinsic and transferable.

3.1.2.4 Similar ratios of TCR-V $\beta-T$ cell subclones in blood and spinal cord of the $\mathrm{HI}$ and the LO-OPT group.

3.1.2.5 The antigen dose-dependent difference in EAE severity still occurs when using a monoclonal $T_{M B P-E M}$ cell line

3.1.2.6 The expression of adhesion-related genes by $T_{M B P}$ cells activated in the lung may be differently regulated by different antigen concentrations

3.2. The role of lung and gut microbiota for i.tr. induced EAE ...................................... 56

3.2.1 The lung hosts a complex microbiota distinct from the gut microbiota ............... 56

3.2.2 Neomycin does not interfere with T cell pathogenicity.................................... 57

3.2.3 Daily administration of neomycin only induces lung inflammation at a dose of $5 \mathrm{mg}$ per day

3.2.4 Neomycin treatment appears to alter the lung microbiota composition in a dose-dependent manner ................................................................................ 59

3.2.5 Neomycin treatment prevents i.tr. induced EAE..............................................63 63

3.2.6 Neomycin treatment per os does not appear to affect i.tr. induced EAE.............. 65

3.2.7 Activation of $T_{M B P-E M}$ cells in the lungs of neomycin-treated rats is not impaired.. 66

3.2.8 Intrapulmonary treatment with neomycin blocks EAE independent of the site

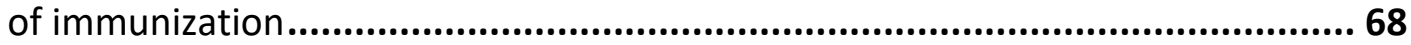

3.2.9 Intrapulmonary treatment with neomycin ameliorates passive transfer EAE ....... 71

3.2.10 Neomycin treatment does not affect blood-brain barrier integrity and endothelial expression of adhesion molecules.................................................. 72

3.2.11 Neomycin treatment may affect microglia function........................................ 73

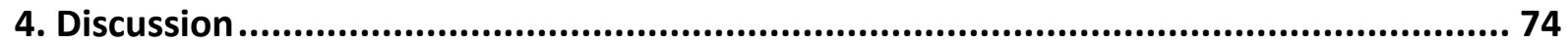

4.1 Establishment and characterization of an i.tr. induced EAE model ........................... 74

4.2 Effect of intrapulmonary antibiotic treatment on EAE ............................................ 78

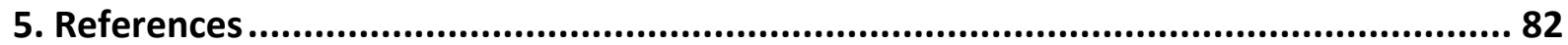

\section{Acknowledgments}




\section{List of Figures}

Figure 1: $\quad$ The different phases of MS ...................................................................... 2

Figure 2: $\quad$ Potential primary target antigens in multiple sclerosis ............................... 5

Figure 3: $\quad$ Generation and passive transfer of autoreactive T cells ............................ 6

Figure 4: The route of autoreactive T cells from injection to CNS infiltration ........... 7

Figure 5: Gating strategy for flow cytometry acquisition of different immune cell populations ........................................................................................ 28

Figure 6: $\quad$ Gating strategy for fluorescence-activated sorting of GFP ${ }^{+} \mathrm{T}_{\mathrm{MBP}}$ cells and spinal cord endothelial cells ...................................................................29

Figure 7: $\quad$ Titration of the MBP concentration used for i.tr. immunization .................33

Figure 8: $\quad$ Titration of the CFA concentration used for i.tr. immunization ..................34

Figure 9: I.tr. immunization of memory rats with a low MBP dose ............................35

Figure 10: Experimental scheme for the kinetic of $T_{M B P}$ cell distribution during i.tr. induced $E A E$

Figure 11: Characterization of $\mathrm{T}_{\mathrm{MBP}}$ cell distribution in different organs during i.tr. induced $E A E$

Figure 12: Kinetic of cytokine expression in $T_{M B P}$ cells during i.tr. induced $E A E$

Figure 13: Kinetic of cytokine expression in lung and spinal cord during i.tr. induced $\mathrm{EAE}$

Figure 14: Regulation of the gene expression profile of $T_{M B P}$ cells following i.tr. activation

Figure 15: $\quad T_{M B P}$ cells stimulated in vitro by lung-derived antigen-presenting cells are encephalitogenic

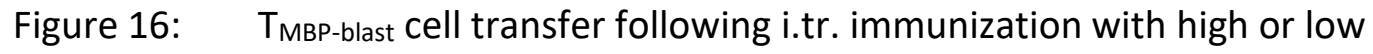
concentrations of a non-self antigen

Figure 17: Proliferation and activation of $\mathrm{T}_{\text {MBP }}$ cells previously activated in the lung with high or low MBP concentrations

Figure 18: Re-transfer of $T_{M B P}$ cells from rats i.tr. immunized with high or low MBP concentrations

Figure 19: $\quad$ Ratio of different TCR-V $\beta$-T cell subclones in blood and CNS of rats i.tr. immunized with high or low MBP concentrations 51

Figure 20: Characterization of $\mathrm{TCR}^{\mathrm{MBP}}$-transgenic $\mathrm{T}$ cell distribution in different organs during i.tr. induced EAE 
Figure 21: Antigen dose-dependent integrin- and chemokine-receptor expression of $T_{M B P}$ cells from blood before CNS infiltration

Figure 22: Comparison between lung and gut microbiota composition 56

Figure 23: Effect of neomycin on $\mathrm{T}_{\mathrm{MBP}}$ cell proliferation and pathogenicity 58

Figure 24: Quantification of immune cells in the lung following neomycin treatment

Figure 25: Effect of i.tr. neomycin treatment on lung and gut microbiota composition 60

Figure 26: Effect of i.tr. neomycin treatment on lung and gut microbiota genera and $\alpha$-diversity

Figure 27: Characterization of the effect of i.tr. neomycin treatment on i.tr. induced $E A E$ 64

Figure 28: Characterization of the effect of p.o. neomycin treatment on i.tr. induced EAE 66

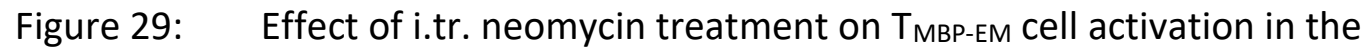
lung

Figure 30: Examination of the relevance of treatment and immunization site for the neomycin effect on $\mathrm{EAE}$

Figure 31: Examination of the relevance of treatment and immunization site for the neomycin effect on $T_{M B P}$ cell distribution 70

Figure 32: Passive transfer EAE in neomycin-treated rats. 71

Figure 33: Effect of i.tr. neomycin treatment on BBB integrity and expression of adhesion molecules 72

Figure 34: Effect of i.tr. neomycin treatment on microglia-typical gene expression ...73 


\section{List of Tables}

Table 1: $\quad$ List of antibodies used for cell staining .................................................14

Table 2: $\quad$ Primer-sequences of 165 rRNA V3 - V4 region ..........................................24

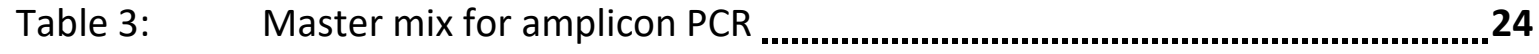

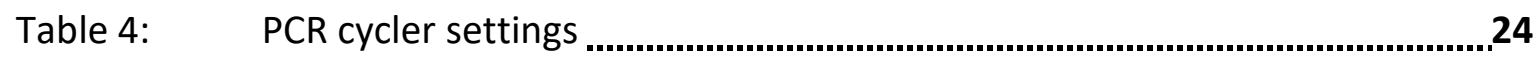

Table 5: $\quad$ List and sequences of qPCR primers ...................................................... 


\section{List of Abbreviations}

\begin{tabular}{|c|c|}
\hline ACK & Ammonium-chloride-potassium \\
\hline $\mathrm{aEAE}$ & Active experimental autoimmune encephalomyelitis \\
\hline APC & Antigen-presenting cell \\
\hline ASV & Amplicon sequence variants \\
\hline BAL(F) & Bronchoalveolar lavage (fluid) \\
\hline BALT & Bronchus-associated lymphoid tissue \\
\hline BBB & Blood-brain barrier \\
\hline bp & Base pairs \\
\hline CCLX & C-C chemokine ligand $x$ \\
\hline $\operatorname{CCR} x$ & C-C chemokine receptor $x$ \\
\hline $\mathrm{CDx}$ & Cluster of differentiation $x$ \\
\hline CFA & Complete Freund's Adjuvant \\
\hline $\mathrm{CIS}$ & Clinically isolated syndrome \\
\hline CMV & Cytomegalovirus \\
\hline CNS & Central nervous system \\
\hline Con A & Concanavalin A \\
\hline COPD & Chronic obstructive pulmonary disease \\
\hline CTRL & Control \\
\hline CXCRx & C-X-C chemokine receptor $x$ \\
\hline $\mathrm{D} x$ & Day $x$ \\
\hline DMEM & Dulbecco's Modified Eagle Medium \\
\hline DMSO & Dimethyl sulfoxide \\
\hline DNA & Deoxyribonucleic acid \\
\hline EAE & Experimental autoimmune encephalomyelitis \\
\hline EBV & Epstein-Barr virus \\
\hline EDTA & Ethylenediaminetetraacetic acid \\
\hline (e)GFP & (Enhanced) green fluorescent protein \\
\hline $\mathrm{EH}$ & Eagle's HEPES medium \\
\hline EM & Effector memory \\
\hline FACS & Fluorescence-activated cell sorting \\
\hline FCS & Fetal calf serum \\
\hline FM & Freezing medium \\
\hline FSC & Forward scatter \\
\hline GA & Glatiramer acetate \\
\hline GPE & GP+E86 packaging cell \\
\hline GWAS & Genome-wide association studies \\
\hline $\mathrm{h}$ & Hour(s) \\
\hline $\mathrm{HI}$ & High concentration of myelin basic protein \\
\hline HLA & Human leukocyte antigen \\
\hline
\end{tabular}


ICAM-1

IFA

IFN $\gamma$

IL-x(R)

Ing LN

i.tr.

i.v.

LFA-1

LO-OPT

LO-SUB

LPS

LSM1077

$\mathrm{N}$

NAI

$\mathrm{Nd}$

NEO

NGS

MACS

MAG

MBP

Med LN

MHC

min

MOG

mRNA

MS

PLP

p.o.

PPMS

OUT

OVA

PBS

PCR

PFA

p.i.

p.t.

ptEAE

qPCR

RM

RNA

rpm

RRMS
Intercellular adhesion molecule 1

Incomplete Freund's Adjuvant

Interferon gamma

Interleukin-x (Receptor)

Inguinal lymph nodes

Intratracheal / intratracheally

Intravenous / intravenously

Lymphocyte function-associated antigen 1

Low-optimal concentration of myelin basic protein

Low-suboptimal concentration of myelin basic protein

Lipopolysaccharide

Lymphocyte Separation Medium 1077

Number of animals

Naïve

Not determined

Neomycin

Next-generation sequencing

Magnetic cell separation

Myelin-associated glycoprotein

Myelin basic protein

Mediastinal lymph nodes

Major histocompatibility complex

Minutes

Myelin oligodendrocyte glycoprotein

messenger ribonucleic acid

Multiple sclerosis

Proteolipid protein

Per os

Primary progressive multiple sclerosis

Operational taxonomic units

Ovalbumin from chicken egg white

Phosphate-buffered saline

Polymerase chain reaction

Paraformaldehyde

Post-immunization

Post-transfer

Passive transfer experimental autoimmune encephalomyelitis

Quantitative real-time polymerase chain reaction

Re-stimulation medium

Ribonucleic acid

Rounds per minute

Relapsing-remitting multiple sclerosis 


$\begin{array}{ll}\text { rRNA } & \text { Ribosomal ribonucleic acid } \\ \text { RT } & \text { Room temperature } \\ \text { S.C. } & \text { Subcutaneous / subcutaneously } \\ \text { SEM } & \text { Standard error of the mean } \\ \text { SPMS } & \text { Secondary progressive multiple sclerosis } \\ \text { S1P(R) } & \text { Sphingosine-1-phosphate (receptor) } \\ \text { TAE } & \text { Tris-acetate-EDTA buffer } \\ \text { TAL } & \text { Transcriptome and Genome Analysis Laboratory } \\ \text { TCGF } & \text { T cell growth factor medium } \\ \text { TCM } & \text { T cell medium } \\ \text { TCR } & \text { T cell receptor } \\ \text { TMBP cell } & \text { Myelin basic protein-specific T cell } \\ \text { TMBP-blast cell } & \text { in vitro activated myelin basic protein-specific T cell } \\ \text { TMBP-EM cell } & \text { Effector memory myelin basic protein-specific T cell } \\ \text { TMBP-migratory cell } & \text { Migratory myelin basic protein-specific T cell } \\ \text { TG } & \text { Transgenic } \\ \text { Thx cell } & \text { T helper } x \text { cell } \\ \text { TM } & \text { Thawing medium } \\ \text { TNF } \alpha & \text { Tumor necrosis factor alpha } \\ \text { UMG } & \text { University Medical Center Göttingen } \\ \text { V } \alpha / \text { V } \beta & \text { Variable } \alpha \text { - or } \beta \text {-chain of the T cell receptor } \\ \text { VCAM-1 } & \text { Vascular cell adhesion protein 1 } \\ \text { VLA-4 } & \text { Very late antigen-4 } \\ \text { WT } & \text { Wild type } \\ \text { XTR-LO } & \text { Extremely low concentration of myelin basic protein } \\ x=\text { varying numbers } & \\ & \end{array}$




\section{Summary}

The lung was recently identified as a central site of $T$ cell reprogramming in experimental autoimmune encephalomyelitis (EAE), a rodent model of multiple sclerosis (MS). While residing in the pulmonary tissue, central nervous system (CNS) antigen-reactive T cells become equipped with the potential to transgress the blood-brain barrier and induce CNS autoimmune disease. In order to better understand the involvement of the lung in the development and progression of EAE, we established an acute EAE model induced by intratracheal (i.tr.) immunization with the myelin antigen myelin basic protein (MBP). Effector-memory $T$ cells reactive against MBP were activated within the lung tissue and induced severe clinical signs of EAE. The activation process was highly efficient: antigen doses about 100 to 1000 times lower than used for subcutaneous (s.c.) immunization were sufficient to trigger MBP-specific T cells to induce EAE. This effect was reproducible in animals which carry low numbers of neonatally transferred MBP-specific memory T cells embedded in their immune repertoire and lung. These observations could be of relevance in context of MS: Minute amounts of pathogenic epitopes resembling self-antigens in the airways of patients with a matching HLA haplotype expression could be sufficient to induce the activation of dormant autoreactive T cells in a process referred to as molecular mimicry, and consequently trigger disease.

In order to identify local factors that can potentially contribute to the efficiency of the lung in triggering autoimmunity, we focused on the recently identified lung microbiota. Intratracheal administration of a locally acting antibiotic induced changes in the lung microbiota composition and significantly reduced EAE. The treatment did neither interfere with $\mathrm{T}$ cell activation within the lung nor change the expression profile of the transferred $T$ cells. Moreover, EAE amelioration was also observed following s.c. immunization and in a transfer EAE model using in vitro activated $T$ cells, suggesting that a distal rather than a local lungintrinsic mechanism could be causative. We observed that i.tr. antibiotic treatment induced a reduced $\mathrm{MHCll}$ expression in the $\mathrm{CNS}$, potentially indicative of a functional impairment in the capacity of microglia or local macrophages to present antigen and consequently trigger CNS inflammation.

Taken together, we observed that the lung represents a highly efficient environment for the activation of autoaggressive T cells and the initiation of CNS autoimmunity, and the lung microbiota appears to be involved in its regulation. How exactly this crosstalk between lung and CNS is mediated remains to be further investigated. 


\section{Introduction}

\subsection{Multiple sclerosis (MS)}

\subsubsection{Clinical picture and epidemiology of MS}

Multiple sclerosis (MS) is a chronic autoimmune disease of the central nervous system (CNS). It is characterized by focal inflammatory demyelinating lesions resulting in axonal loss and neurodegeneration. The clinical manifestation is multifaceted and dependent on the CNS area affected. Hallmark symptoms include numbness and tingling, fatigue, mobility problems, and impairments of vision and cognition (Compston and Coles, 2008).

Principally, four types of MS differing in their clinical course can be distinguished (Lublin and Reingold, 1996): clinically isolated syndrome (CIS), relapsing-remitting MS (RRMS), primary progressive MS (PPMS) and secondary progressive MS (SPMS). About $80-85 \%$ of patients are initially diagnosed with RRMS, characterized by sudden relapses with subsequent episodes of remission. Impairments occurring during relapses either completely or only partially resolve, the latter accounting for $40 \%$ of RRMS attacks with an increasing probability over time. RRMS usually starts with a CIS, an attack indicative of demyelination without fulfilling MS criteria. While in some patients a CIS remains a unique episode, between 30 and $70 \%$ develop MS later in life. Around 20 years after disease onset, RRMS slowly converts into SPMS in around 50-65\% of patients. In SPMS, neurological deficits start worsening progressively while acute attacks and intervals of remission fade. PPMS affects $10-20 \%$ of patients. Following initial symptoms, disability progresses steadily in absence of acute attacks and remission periods. The age of onset of PPMS is around 40 years, which corresponds to the average age of conversion from RRMS to SPMS (Fig. 1; Weinshenker et al., 1989; Lublin and Reingold, 1996; Miller et al., 2005; Miller and Leary, 2007; Compston and Coles, 2008).

With around $2-2.5$ million people affected worldwide and an age of onset between 20 and 50 years old, $\mathrm{MS}$ is the most common immune-mediated disease of the central nervous system (CNS) and disabling neurological condition among young adults (Compston et al., 2006; World Health Organization, 2008). MS is particularly common among Caucasians and affects women around twice as often as men (Compston and Coles, 2008; Milo and Kahana, 2010). 


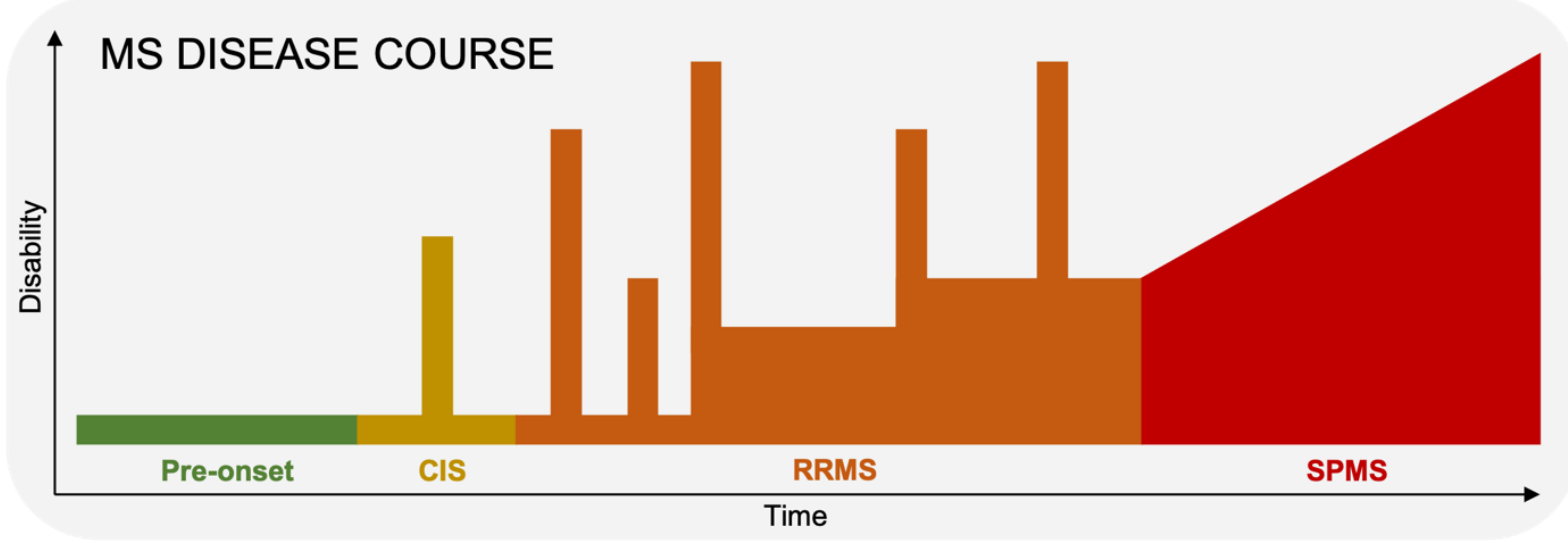

Figure 1: The different phases of MS. Depicted is an exemplified typical course of MS. In the pre-onset phase, the disease starts manifesting itself on a subclinical level. The patients are free of symptoms. Increasing immune cell infiltration and CNS damage at some point provoke a first attack typically lasting about $24 \mathrm{~h}$ and often starting with tingling or numbness of e.g. face or fingertips or a blurry vision. These patients are usually initially diagnosed with a clinically isolated syndrome (CIS). While some patients fully recover without experiencing further relapses throughout life, CIS in many patients converts to clinically definite MS. For several years, patients experience sudden acute attacks with subsequent remission (RRMS). Initially, symptomatic remission is more or less complete. Over time, however, remission starts becoming incomplete and the symptoms start lingering, resulting in increasing disability. Around 20 years after onset, RRMS oftentimes converts into a secondary-progressive course (SPMS) characterized by a progressive worsening of neurological deficits with few or no acute attacks or episodes of remission. Some MS patients also have a primary progressive disease course (PPMS) without acute attacks or episodes of remission (not depicted; adapted from Disanto et al., 2012).

\subsubsection{General etiology of MS}

The cause of MS is yet unknown. Though research has led to the identification of a number of treatment targets and the development of a variety of medications, MS to this day is still not curable and life expectancy of patients remains decreased by $5-10$ years compared to unaffected individuals (Compston and Coles, 2008).

$\mathrm{MS}$ is widely believed to be caused by a combination of genetic and environmental risk factors. According to this hypothesis, certain triggers can cause disease in individuals with a genetic predisposition.

\section{Genetic risk factors}

Indications for a genetic component are based on population-based studies that identified an increased risk in close relatives of MS patients which decreases with distance. The highest concordance rate of $25-30 \%$ can be observed for monozygotic twins (Compston and Coles, 2002; Ebers et al., 2000; Robertson et al., 1996). Genome-wide association studies (GWAS) could show that genetic risk factors are often immune-related. Many of the loci found to be associated with MS are located in the human leukocyte antigen (HLA) complex. Genes in this system mainly encode for major histocompatibility complex (MHC) proteins, components of the complement system and other factors of immunological relevance. The HLA allele $D R B 1^{*} 15: 01$ is often referred to as the primary MS risk variant. GWAS found that single nucleotide polymorphisms in this gene show a strong association with MS. Other loci described to be associated with an increased MS risk include genes encoding for cytokine receptors (IL-2RA, IL-7RA) and costimulatory molecules (B7-1, B7-2), indicating that genetic predisposition commonly goes hand in hand with an immunological dysfunction (Baranzini, 
2011; Compston and Coles, 2008; International Multiple Sclerosis Genetics Consortium et al., 2011).

\section{Environmental risk factors}

Besides genetics, a variety of environmental risk factors has been described to be associated with MS, including childhood obesity, reduced vitamin D levels as a result of insufficient sunlight exposure in the Northern Hemisphere, a dysregulation of the gut microbiome, smoking, and viral infections (Ascherio, 2013; Ascherio and Munger, 2007a, 2007b; Ascherio et al., 2010; Compston and Coles, 2008).

\subsubsection{Lung-associated MS risk factors}

Due to the relatively large surface of the pulmonary epithelium and the correspondingly intense contact with the environment, the lung is particularly vulnerable. In addition, the lung is equipped with a highly specialized immune system, which aims to control the colonization by pulmonary pathogens. Given these characteristics, it is not surprising that disturbances of this delicate and complex system have been described to affect MS susceptibility, indicating an involvement of the lung in its pathogenesis.

As indicated above, many of the environmental factors observed to be correlated with an increased MS risk are lung-associated. In fact, a variety of inflammatory conditions of the upper respiratory system have been implicated in MS manifestation (Panitch, 1994; Correale et al., 2006). In particular, infections with viruses such as influenza or herpesviruses like Epstein-Barr (EBV) or Cytomegalovirus (CMV) are believed to increase the risk of developing MS and exacerbate its course (Halenius and Hengel, 2014; Lang et al., 2002; Oikonen et al., 2011). Indeed, viral infections of the lung appear to be strongly correlated with an increased relapse rate (Sibley et al., 1985; Buljevac et al., 2002).

Furthermore, cigarette smoking was found to be strongly associated with both an increased disease susceptibility and a more rapid disease progression (Antonovsky et al., 1965; Ghadirian et al., 2001; Hernán et al., 2001; Riise et al., 2003; Pekmezovic et al., 2006; Ascherio and Munger, 2007b; Hedström et al., 2009, 2013; Jafari and Hintzen, 2011). Compared to nonsmokers, smokers have an approximately 1.2 to 1.8 -fold increased relative risk to develop MS (Riise et al., 2003; Hawkes, 2007). Additionally, MS patients that started smoking early in life tend to develop a chronic clinical course more frequently and after a shorter disease duration, and their risk of disability progression is significantly increased (Sundström and Nyström, 2008). Similar to smoking, the often consequential chronic obstructive pulmonary disease (COPD) has been shown to correlate with an increased MS-risk (Egesten et al., 2008). Together, these observations suggest a central role of the lung in shaping CNS autoimmunity. 


\subsubsection{Pathogenesis of MS}

A clear mechanistic basis of the pathogenesis underlying MS remains to be unraveled. However, multiple lines of evidence suggest that auto-aggressive CNS-reactive effector T lymphocytes are the culprits of MS (Compston and Coles, 2002, 2008; Kebir et al., 2007; Shi et al., 2007; Tzartos et al., 2008; Viglietta et al., 2004). Autoantigen-specific T cells can be found in the immune repertoire of healthy individuals. Usually, these cells are quiescent and harmless for the organism. In contrast, in the context of MS, it is thought that these cells become activated in the periphery and consequently are somehow able to cross the bloodbrain barrier (BBB), a structure that in healthy individuals strongly limits immune cell infiltration into the CNS. Once infiltrated, the T cells cause tissue-damaging inflammatory responses and MS-typical lesions. The hypothesis that $\mathrm{T}$ cells are the major disease-driving factor of MS is strongly supported by the fact that most of the available MS drugs directly or indirectly affect the differentiation, migration or activation of T cells (Baecher-Allan et al., 2018):

Natalizumab for instance is a monoclonal antibody directed against very late antigen-4 (VLA4). Interference with VLA-4 prevents activated T cells from adhering to and transgressing through the BBB. Another MS drug targeting $T$ cells is Fingolimod. Treatment with this immunomodulatory drug induces the internalization of sphingosine-1-phosphate (S1P) receptors, thereby preventing potentially autoreactive $T$ cells from exiting the lymph nodes and entering the CNS. Glatiramer acetate (GA) is another immunomodulatory medication commonly used to treat MS. GA is a random copolymer composed of four amino acids also found in myelin basic protein (MBP). It can bind to MHC class II molecules including HLA-DR2, thereby inhibiting the activation of MBP-specific autoreactive T cells (O'Brien et al., 2010). Despite strong indications that T cells play a key role for the initiation and progression of MS, it remains to be clarified where, when and how auto-reactive $T$ cells are activated and equipped with the potential to overcome the BBB and infiltrate the CNS.

\subsection{Experimental autoimmune encephalomyelitis (EAE)}

Today's understanding of MS pathogenesis is to a high degree based on studies in the main MS animal model, experimental immune encephalomyelitis (EAE). In this model, myelinreactive $C D 4^{+} \mathrm{T}$ cells are the definite cellular cause of disease. The history of EAE can be traced back to the early 1930s, when Thomas Milton Rivers immunized Rhesus monkeys with rabbit CNS homogenate and subsequently observed the formation of perivascular demyelinating lesions reminiscent of MS pathophysiology (Rivers et al., 1933). Thereby, he could show that a peripheral immune response can cause CNS autoimmunity. Ever since, EAE has steadily been refined and induced in a variety of animals including guinea pigs, rabbits and non-human primates. Today, EAE is well-established and most commonly used in rodents like mice and rats (Mannie et al., 2009; Miller et al., 2010). In most of the cases, EAE in these animals is clinically characterized by an ascending paresis starting with weight loss and a reduced tail tonus and developing into complete paralysis of tail and hind limbs at the peak of disease. 
EAE can be induced in two ways; actively by immunization with myelin antigens or passively by transfer of myelin-reactive $\mathrm{CD} 4^{+} \mathrm{T}$ cells.

\subsubsection{Active EAE (aEAE)}

To induce active EAE ( $\mathrm{AEAE}$ ), animals are immunized with myelin antigens such as myelin basic protein (MBP), myelin oligodendrocyte glycoprotein (MOG) or proteolipid protein (PLP) mixed with an adjuvant (Fig. 2; Lipton and Freund, 1953; Mannie et al., 2009; Miller et al., 2010). Upon encounter with their cognate antigen presented by professional antigen-presenting cells (APCs), potentially autoreactive naïve T cells are primed and differentiate into effector T cells in lymph nodes draining the site of immunization. After several rounds of expansion, the cells exit the lymph nodes, enter the circulation and manage to overcome the BBB. In the CNS, they are reactivated by local APCs presenting their cognate antigen. Upon reactivation, $T$ cells release proinflammatory cytokines and chemokines which leads to a massive recruitment of immune cells including lymphocytes and monocytes / macrophages, thereby triggering a cascade of inflammation, demyelination and local damage causing the clinical symptoms described above (Mix et al., 2010).

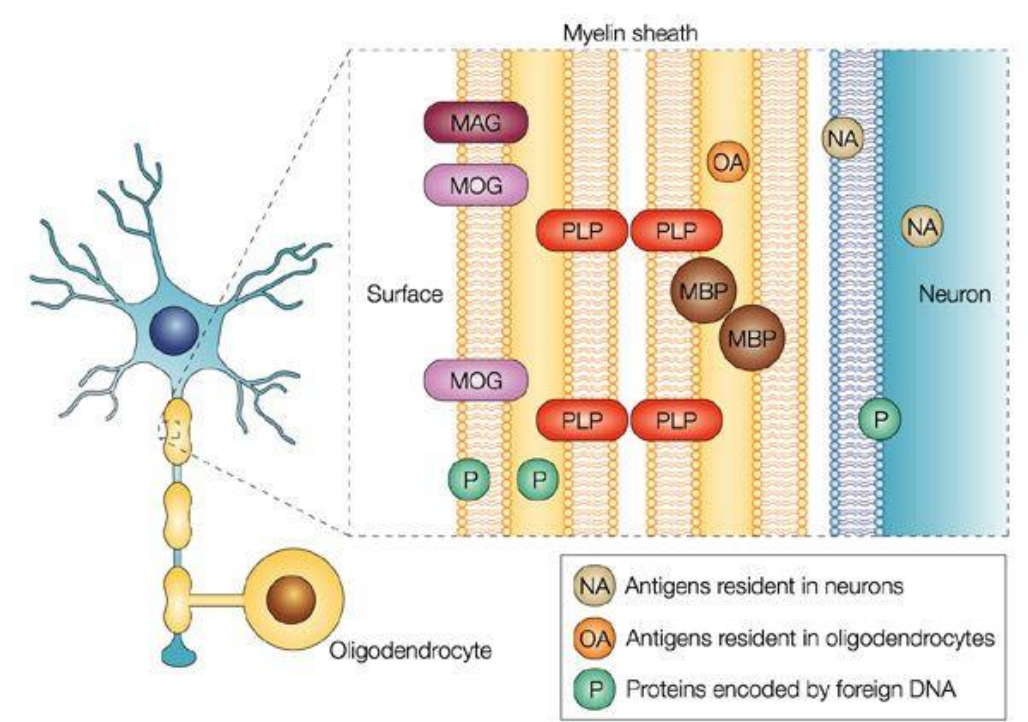

Figure 2: Potential primary target antigens in multiple sclerosis. T cells reactive against myelin-associated glycoprotein (MAG), myelin basic protein (MBP), myelin oligodendrocyte glycoprotein (MOG) and proteolipid protein (PLP) are frequently identified in the immune repertoire of MS patients. MBP, MOG and PLP are commonly used as encephalitogenic antigens to induce EAE in different rodent models. (Adapted from Hemmer et al., 2002).

\subsubsection{Passive transfer EAE (ptEAE)}

In the passive transfer EAE (ptEAE) model, myelin-reactive $C D 4^{+} \mathrm{T}$ cells are isolated from the draining lymph nodes of actively-immunized animals before EAE onset. After in vitro activation, the cells are transferred into naïve recipient animals. After an asymptomatic period of $3-4$ days, the cells invade the CNS, causing neurological disease as described above for aEAE (Fig. 3). The fact that $T$ cell transfer alone is sufficient to induce CNS autoimmunity 
proved their crucial role as culprits in EAE and potentially in MS (Paterson, 1960; Ben-Nun et al., 1981). The ptEAE model has a key advantage: CNS-reactive CD4 ${ }^{+} \mathrm{T}$ cells isolated from immunized animals can be retrovirally transduced to express fluorescent markers, allowing to track and functionally characterize the cells at any point on their route into the CNS of recipient animals (Flügel et al., 1999).

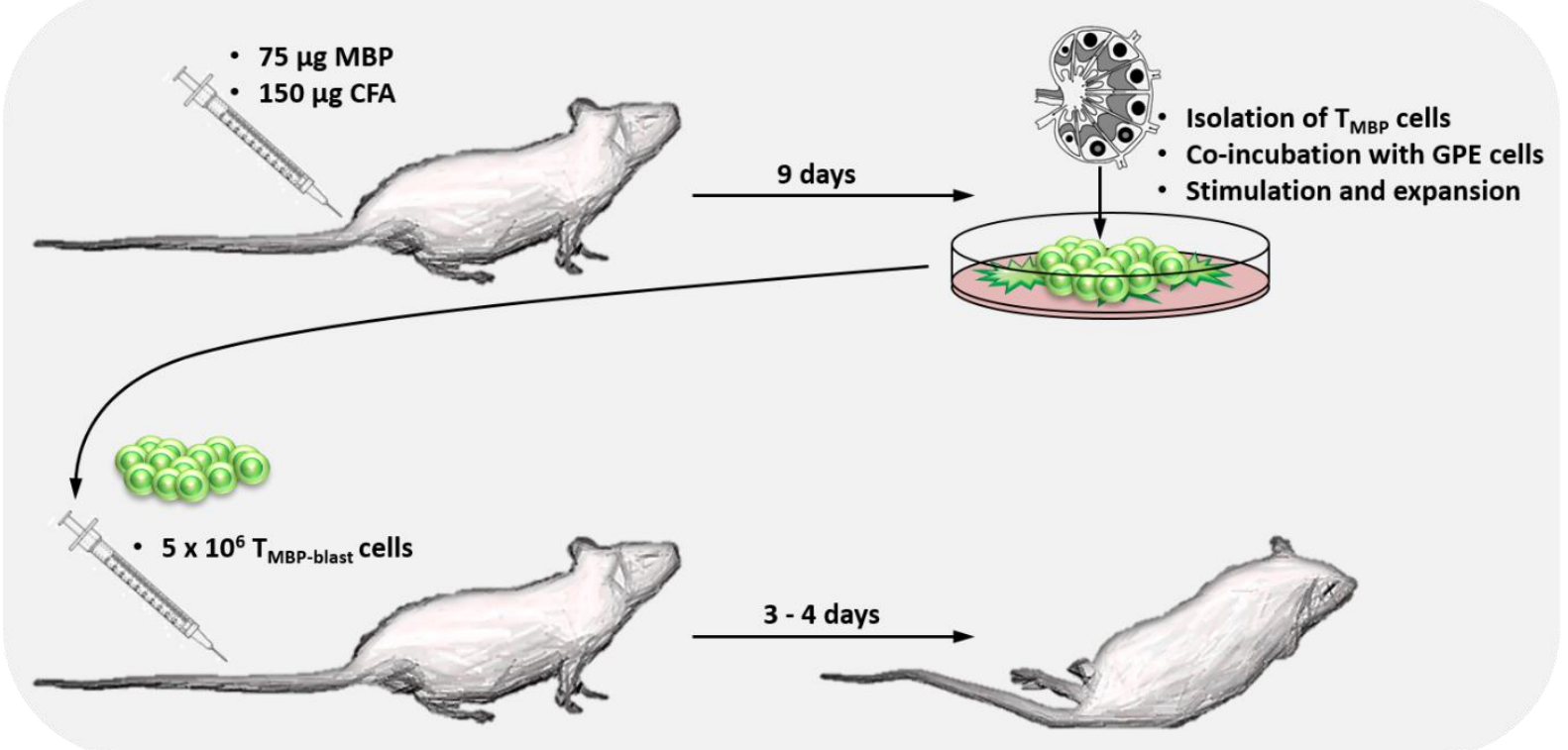

Figure 3: Generation and passive transfer of autoreactive T cells. Rats are subcutaneously immunized with myelin basic protein (MBP) emulsified in Complete Freund's Adjuvant (CFA). Nine days post-immunization, draining lymph nodes are isolated and brought to single cell suspension. The lymph node cells including MBP-reactive $T\left(T_{M B P}\right)$ cells are co-cultured with GP+E86 (GPE) packaging cells. Thereby, the T cells are retrovirally transduced to express GFP. The T cells are stimulated with $M B P$, resulting in their activation. Adding IL-2 on D2 post-stimulation leads to proliferation and expansion of the cells, before they again become resting around 6 days post-stimulation. On D7 post-stimulation, the cells can again be re-stimulated. This cycle of T cell stimulation and expansion can usually be repeated $5-6$ times. On D2 post-stimulation, the fully activated $T_{M B P}$. blast cells can be adoptively transferred into naïve recipient animals, resulting in the induction of EAE after $3-4$ days.

\subsubsection{The lung as a critical site of $T$ cell reprogramming in EAE}

Using the ptEAE model in the Lewis rat (Paterson, 1960; Ben-Nun et al., 1981; Flügel et al., 1999), it could be observed that fluorescently-labelled MBP-reactive T cells previously activated in vitro ( $\mathrm{T}_{\mathrm{MBP} \text {-blast }}$ cells) vanished from the circulation of recipient animals within minutes following intravenous (i.v.) transfer. Neither could they be found in the blood, nor did they home to secondary lymphoid organs like lymph nodes or spleen. Only around 48 hours post-transfer (p.t.), the cells started reappearing in blood and spleen, just before entering the CNS. When examining the transferred cells' location during the prodromal phase, Odoardi and colleagues (2012) observed the cells to home to the lung shortly upon transfer. Within the first $12 \mathrm{~h}$, they mainly resided in the peripheral lung tissue. About $24-48 \mathrm{~h}$ p.t., the cells migrated along bronchial structures and accumulated in bronchus-associated lymphoid tissues (BALT). About $48 \mathrm{~h}$ p.t., just before their reappearance in blood and spleen, they were found in high numbers in the lung-draining mediastinal lymph nodes (Odoardi et al., 2012). Strikingly, while residing in the lung, their gene-expression profile was fundamentally reprogrammed: while transcription of genes involved in T cell activation and proliferation was 
decreased, expression of genes relevant for T cell motility, cell adhesion and migration was highly increased (Fig. 4; Odoardi et al., 2012).

In addition, the transferred $\mathrm{T}_{\mathrm{MBP} \text {-blast }}$ cells changed their responsiveness to migration stimuli, explaining their reorientation within the lung tissue. Initially, the freshly immigrated T cells responded to homeostatic chemokines (CCL19 and -21), which are mainly expressed in the respiratory tract and BALT. On their way, the T cells upregulated S1PR1, a sphingolipidresponsive receptor that controlled the egress of cells from the lung. Furthermore, inflammatory chemokine receptors (CCR5 and CXCR3) were upregulated, facilitating T cell attachment to the CNS endothelium (Odoardi et al., 2012). Thus, while residing in the lung, the transcription profile of transferred $T_{M B P}$-blast cells was switched from an activatory to a

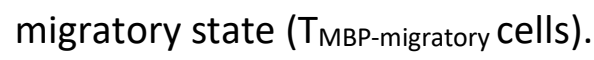
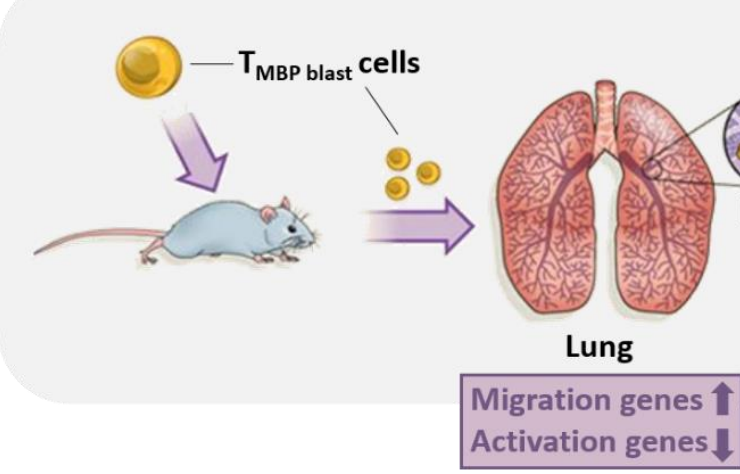

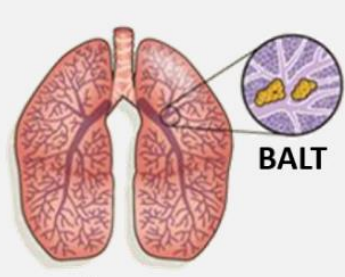

BALT

\section{.}

Figure 4: The route of autoreactive T cells from injection to CNS infiltration. Within minutes following adoptive transfer, $\mathrm{T}_{\mathrm{MBP} \text {-blast }}$ cells started homing to the lung. While residing within the pulmonary environment, the cells switched their gene expression profile from an activatory to a migratory state. Around two days post-transfer, the cells started migrating into the lung-draining mediastinal lymph nodes (not depicted) before reappearing in blood circulation and spleen around D3 after transfer, just before CNS infiltration. The residence and reprogramming in the lung were prerequisite for the cells to transgress the BBB and enter the CNS (adapted from Ransohoff, 2012).

This profound reprogramming in the lung was found to be substantial for the ability of the T cells to enter the CNS, which could further be confirmed in an anastomotic experimental setup: $48 \mathrm{~h}$ after $T_{M B P-b l a s t}$ cell transfer, the blood circulations of the recipient and a naïve rat were conjoined. The transferred cells at that point had already been reprogrammed in the recipient animal's lung. The TMBP-migratory cells passing through the shunt within $12 \mathrm{~h}$ of anastomosis started infiltrating the conjoined naïve rat's CNS in similar frequencies and at the same time as in the recipient rat, thereby excluding a CNS preconditioning. Moreover, $\mathrm{T}_{\mathrm{MBP} \text { - }}$ migratory cells isolated from spleens of $T_{\text {MBP-blast }}$ cell recipients $60 \mathrm{~h}$ post-transfer readily migrated to and infiltrated the CNS of naïve animals shortly after re-transfer (Odoardi et al., 2012).

The stimulatory potential of the lung could further be demonstrated when intratracheally (i.tr.) immunizing so-called memory animals with MBP. To generate these memory animals, rats are neonatally transferred with memory $T_{M B P}$ cells. When injected until postnatal D2 - 3, these cells are tolerated by and embedded into the recipient animals' immune system. They persist lifelong in low numbers in lymph nodes, spleen, and notably also in the lung (Kawakami et al., 2005). Upon i.tr. immunization of the adult memory animals, the memory $T_{\text {MBP }}$ cells became activated and strongly proliferated in lung and mediastinal lymph nodes. Subsequently, the cells egressed the lung environment and entered the blood stream before 
infiltrating the CNS and triggering EAE (Odoardi et al., 2012). Taken together, these findings clearly demonstrate the relevance of the lung as a critical site of stimulation and acquisition of pathogenicity of auto-reactive T cells in a model of CNS autoimmunity, suggesting a potentially similar role of the lung in the context of MS.

\subsection{Potential mechanistic links between lung and CNS autoimmunity}

Until recently, the lung had not been considered to be of immunological relevance for the trafficking of autoaggressive T cells to the CNS. The identification of the lung as the central checkpoint of T cell reprogramming in its animal model EAE was surprising (Odoardi et al., 2012). The observation that in EAE a transient residence in the pulmonary environment appears to be a prerequisite for CNS-reactive T cells to infiltrate the CNS now raises the question of whether and how the lung might be of similar relevance in the context of MS.

As mentioned earlier, many of the environmental factors described to be associated with MS suggest an implication of the lung in its pathogenesis. Most studies correlating inflammatory lung conditions with an increased MS risk are of descriptive nature and lack a mechanistic basis. Potential mechanisms include a bystander activation of normally quiescent CNSreactive $T$ cells under chronic inflammatory conditions. Moreover, in the context of lung infections, viral or bacterial epitopes presented to T cells by professional APCs could share an identical or similar peptide sequence with certain CNS proteins. Potentially CNS-reactive T cells activated when encountering the pathogenic antigen in the lung could falsely be activated and trigger an autoimmune response against akin self-antigens in the CNS, a concept referred to as molecular mimicry (Lang et al., 2002; Wucherpfennig and Strominger, 1995).

In line with chronic obstructive conditions or infections of the lung, a potentially consequential dysregulation of the local microbiome may contribute to an altered MS susceptibility. Indications stem from studies focused on the gut microbiota. Given its significant contribution to the development and maintenance of the immune system, the composition of the microbial intestinal flora has recently been extensively discussed as an important component in the development of autoimmune diseases (Jangi et al., 2016; Ochoa-Repáraz et al., 2009). Its importance could already be demonstrated in the MS animal model EAE. For instance, the oral treatment of mice with a broad-spectrum of antibiotics, resulting in a reduction of the gut microbiota, could significantly ameliorate the development of EAE in two different mouse strains, SJL and C57BL/6 (Ochoa-Repáraz et al., 2009). The authors observed the protective effect to be associated with a reduced expression of pro-inflammatory and an increased expression of anti-inflammatory cytokines and suggested a role of regulatory $T$ cells for the maintenance of peripheral immune homeostasis (Ochoa-Repáraz et al., 2009).

In line with this observed effect, raising mice in sterile conditions could be shown to completely prevent disease in a spontaneous relapsing-remitting SJL mouse model of EAE (Berer et al., 2011; Goverman et al., 1993). In these transgenic mice, the majority of CD4 ${ }^{+} \mathrm{T}$ cells expresses a transgenic T cell antigen receptor (TCR) specific for MOG peptide $92-106$. When rearing the mice under specific pathogen-free conditions, about $80 \%$ of these animals 
spontaneously developed EAE within 3 to 8 months. Rearing these mice in germ-free conditions, however, completely protected the animals from developing EAE. Moreover, mice kept in germ-free conditions for 6-12 weeks promptly developed EAE upon recolonization with conventional microbiota, indicating that germ-free rearing per se did not induce a general immune deficiency and that in deed the absence of a commensal microbiota protected the mice from developing disease (Berer et al., 2011). Along with more and more emerging lines of evidence, these findings indicate a crucial role of the gut microbiota in the regulation of MS development (Chu et al., 2018; Joscelyn and Kasper, 2014). Gut microbiota dysbiosis is suggested to increase MS risk and was already found to be associated with the systemic autoimmune disease rheumatoid arthritis (Budhram et al., 2017; Zhang et al., 2015), as well as neurodegenerative disease like Alzheimer's disease and Parkinson's disease (Parashar and Udayabanu, 2017; Westfall et al., 2017).

For a long time, the lung was thought to be sterile under physiological conditions. Owing to ongoing advancements in the development of molecular techniques for the investigation of bacterial communities, however, it could recently be shown that the lung is indeed colonized by a complex microbial flora. Like in the gut, the predominant phyla colonizing the healthy lung are the Gram-negative Bacteriodetes and Gram-positive Firmicutes. Prominent genera abundant in the healthy lung are Prevotella, Veillonella, Streptococcus and Pseudomonas (Dickson et al., 2013, 2014). Despite some similarities, the microbiota composition of gut and lung in general differ significantly (Dickson and Huffnagle, 2015). This can easily be attributed to the very distinct physiological characteristics of both environments: the gastrointestinal tract harbors a variety of different compartments as well as physical and chemical barriers. The stomach for instance is acidic with a $\mathrm{pH}$ of around 2 whereas the duodenum is alkaline with a $\mathrm{pH}$ of around 8 . Consequently, the microbiota composition varies between the different gastrointestinal compartments but is generally stable. Due to the lack of a real physical barrier between lung and the environment, the microbiota of the lung is more dynamic and transient compared to the gut. Another obvious difference between gut and lung is the bacterial density: microbiota colonizing the gut outnumber those found in the lung by orders of magnitude (Dickson and Huffnagle, 2015; Scales et al., 2016; Dang and Marsland, 2019).

While research focused on the role of the gut and its microflora in regulating autoimmune diseases has already entered the focus of researchers worldwide, little in this context is yet known about the lung and its microbiota. Dysbiosis of the lung microbiota has been described in a variety of patients with inflammatory and obstructive conditions of the lung including the MS risk factors COPD and cigarette smoking (Charlson et al., 2010; Hilty et al., 2010). Given that growing evidence points toward an implication of the lung as a key player in the pathogenesis of MS, understanding how the local microflora could be linked to MS susceptibility is of critical relevance. 


\subsection{Aims and objectives}

Aim of my project was to better understand the role of the lung in shaping CNS autoimmunity. Especially, we wanted to examine its function as a potentially critical site of CNS-reactive $T$ cell activation and EAE initiation. Moreover, we wanted to examine how antibiotic treatment of the commensal lung microbiota may affect clinical aspects of EAE.

\subsubsection{Establishment and characterization of an i.tr. induced EAE model}

In order to examine efficiency and relevance of the lung for T cell activation, we aimed to establish a model based on intrapulmonary activation of MBP-specific T effector memory ( $T_{M B P-E M}$ ) cells by i.tr. immunization. To this end, we first determined the optimal combination of antigen and adjuvant to efficiently induce EAE. Subsequently, we performed an in-depth characterization of the model by determining $T_{M B P}$ cell distribution and activation state in various organs at critical time points post-immunization.

\subsubsection{Effect of intrapulmonary antibiotic treatment on EAE}

Using the established i.tr. induced EAE model, we wanted to test whether an alteration of the lung microbiota composition via local antibiotic treatment might affect EAE in a similar way as described for the gut. To this end, rats were intratracheally treated daily with different doses of Neomycin, a non-absorbable aminoglycoside, for 7 days. Subsequently, inflammation state and microbiota composition of the lung were determined. Moreover, EAE was induced via the lung and the clinical course monitored. After observing that local antibiotic treatment could indeed prevent $E A E$, the Neomycin dose efficiently blocking disease without causing lung inflammation was used to examine the underlying mechanism. 


\section{Materials and Methods}

\subsection{Materials}

\subsubsection{Media and buffers}

All buffers were prepared with water purified using a Milli-Q purification system (Merck, Germany) unless otherwise indicated.

Dulbecco's Modified

Eagle Medium (DMEM)

Eagle's HEPES medium (EH)

Phosphate-buffered saline (10x PBS)

Tris-acetate-EDTA buffer (50x TAE)

T cell medium (TCM)
$13.4 \mathrm{~g} / \mathrm{L}$

DMEM Powder

(Thermo Fisher Scientific, USA)

$3.72 \mathrm{~g} / \mathrm{L} \quad \mathrm{NaHCO}_{3}$

(Roth, Germany)

$75 \%$

$25 \%$

DMEM

HEPES 1M

(Thermo Fisher Scientific, USA)

$\begin{array}{ll}8.1 \mathrm{mM} & \mathrm{Na}_{2} \mathrm{HPO}_{4} \text { (Roth, Germany) } \\ 1.47 \mathrm{mM} & \mathrm{Na}_{2} \mathrm{H}_{2} \mathrm{PO}_{4} \text { (Roth, Germany) } \\ 137 \mathrm{mM} & \mathrm{NaCl} \text { (Roth, Germany) } \\ 2.68 \mathrm{mM} & \mathrm{KCl} \text { (Roth, Germany) } \\ & \text { adjusted to pH 7.2 - 7.4 with HCl } \\ & \text { (Roth, Germany) }\end{array}$

$242 \mathrm{~g}$

Tris Free base

(Thermo Fisher Scientific, USA)

$18.61 \mathrm{~g} \quad \mathrm{Na}_{2} x$ EDTA (Roth, Germany)

$57.1 \mathrm{~mL} \quad$ Glacial Acetic Acid

(Merck, Germany)

ad $1 \mathrm{~L}$

\begin{tabular}{|c|c|}
\hline $950 \mathrm{~mL}$ & DMEM \\
\hline $\mathrm{mL}$ & $\begin{array}{l}\text { Non-essential amino acids } \\
\text { (Thermo Fisher Scientific, USA) }\end{array}$ \\
\hline $\mathrm{mL}$ & $\begin{array}{l}\text { Penicillin / Streptomycin } \\
\text { (Thermo Fisher Scientific, USA) }\end{array}$ \\
\hline $\mathrm{mL}$ & $\begin{array}{l}\text { Sodium pyruvate } \\
\text { (Thermo Fisher Scientific, USA) }\end{array}$ \\
\hline $\mathrm{mL}$ & $\begin{array}{l}\text { L-asparagine monohydrate } \\
\text { (Sigma Aldrich, Germany) }\end{array}$ \\
\hline & $\begin{array}{l}\text { L-glutamine } \\
\text { (PAN Biotech, Germany) }\end{array}$ \\
\hline
\end{tabular}


$4 \mu \mathrm{L} \quad \beta$-mercaptoethanol $\quad(13.6 \mathrm{~mol} / \mathrm{L})$

(Thermo Fisher Scientific, USA)

Re-stimulation medium (RM)

$99 \%$

TCM

$1 \%$

Rat serum (In-house production)

T cell growth factor medium (TCGF)

$85 \%$

TCM

$10 \%$

Horse serum (Merck, Germany)

$5 \%$

Conditioned medium from

Concanavalin A-treated

splenocytes (Con A supernatant;

Rosenberg et al., 1978)

FACS buffer

$2 \mathrm{mM}$

$0.5 \%$

$\mathrm{Na}_{2}$ XEDTA (Roth, Germany)

Albumin Fraction $\mathrm{V}$

(Roth, Germany)

in $1 x$ PBS

Freezing medium (FM)

$80 \%$

$10 \%$

Horse serum (Merck, Germany)

$10 \%$

TCM

DMSO (Roth, Germany)

Thawing medium (TM)

$90 \%$

$\mathrm{EH}$

$10 \%$
Ammonium-chloride-potassium buffer (ACK)
$0.15 \mathrm{~mol} / \mathrm{L} \quad \mathrm{NH}_{4} \mathrm{Cl}$ (Roth, Germany)

$1 \mathrm{mmol} / \mathrm{L} \quad \mathrm{KHCO}_{3}$ (Roth, Germany)

$0.1 \mathrm{mmol} / \mathrm{L} \quad \mathrm{Na}_{2} \mathrm{xEDTA}$ (Roth, Germany)

Adjusted to $\mathrm{pH} 7.2-7.4$ with $\mathrm{HCl}$

(Roth, Germany)

$500 \mathrm{~mL} \quad 1 \times$ DPBS without $\mathrm{CaCl}_{2}$ and $\mathrm{MgCl}_{2}$

(Thermo Fisher Scientific, USA)

$0.5 \% \quad$ Albumin Fraction V

(Roth, Germany)

$15 \mathrm{~mL} \quad \mathrm{EH}$

$0.4 \mathrm{u} / \mathrm{mL} \quad$ Liberase (Roche, Switzerland)

$120 \mathrm{u} / \mathrm{mL}$ DNase I (Roche, Switzerland)

1.45g Nycodenz (Axis-Shield, UK)

$9.5 \mathrm{~mL} \quad 1 \times$ PBS

$14.5 \%$ Nycodenz solution 
Isotonic Percoll (Isopercoll)

Underlay Percoll

$40 \%$ Percoll

$70 \%$ Percoll

\subsubsection{Reagents, chemicals and sera}

Anti-PE MicroBeads

Albumin Fraction $\mathrm{V}$

APC beads (BD Calibrite)

$\beta$-Mercaptoethanol

B. thuringiensis BTI18246 DNA

Chloroform

Diethylether

Dimethyl sulfoxide (DMSO)

DMEM powder

DNase

DPBS (10x) without $\mathrm{CaCl}_{2}$ and $\mathrm{MgCl}_{2}$

Ethidium bromide

Fetal calf serum (FCS)

GeneRuler 1 kb DNA ladder

Glycogen

G-418 sulfate solution

HEPES

Horse serum

Hydrochloric acid $(\mathrm{HCl})$

Incomplete Freund's Adjuvant (IFA)

Isopropanol

Isotonic saline solution $(\mathrm{NaCl}, 0.9 \%)$

Liberase

Low-electroendosmosis (LE)-Agarose

Lymphocyte separation medium 1077

(LSM1077)

Lysozyme from chicken egg
$90 \%$

$10 \%$

$64.22 \%$

$35.78 \%$

Isopercoll

1x PBS

$40 \%$

Isopercoll

$60 \%$

1x PBS

$70 \%$

Isopercoll

$30 \%$

1x PBS
Miltenyi Biotec, Germany

Roth, Germany

BD Biosciences, USA

Roth, Germany

In-house production

Roth, Germany

Roth, Germany

Roth, Germany

Thermo Fisher Scientific, USA

Roche, Switzerland

Thermo Fisher Scientific, USA

Merck, Germany

Merck, Germany

Thermo Fisher Scientific, USA

Roche, Switzerland

Capricorn Scientific, Germany

Thermo Fisher Scientific, USA

Merck, Germany

Roth, Germany

BD Biosciences, USA

Roth, Germany

B. Braun, Germany

Roche, Switzerland

Biozym, Germany

PromoCell, Germany

Serva, Germany 
M. tuberculosis H37Ra

Neomycin

Nycodenz

Orange DNA Loading Dye (6x)

Paraformaldehyde (PFA)

TRI Reagent

Trypsin-EDTA solution (10x)

qPCR Mastermix

\subsubsection{Antigens and adjuvants}

Myelin basic protein (MBP)

isolated from guinea pig brains

Ovalbumin (OVA) from chicken egg white Merck, Germany

Complete Freund's Adjuvant (CFA, stock)

In-house production
BD Biosciences, USA

Thermo Fisher Scientific, USA

Axis-Shield, UK

Thermo Fisher Scientific, USA

Roth, Germany

Merck, Germany

Merck, Germany

Eurogentec, Belgium

$40 \mathrm{mg}$ M. tuberculosis H37Ra

(BD Biosciences, USA)

$10 \mathrm{~mL}$ Incomplete Freund's Adjuvant (IFA)

(BD Biosciences, USA)

\subsubsection{Antibodies}

Table 1: List of antibodies used for cell staining

\begin{tabular}{|l|l|l|l|l|}
\hline Antibody & Fluorochrome & Clone & Dilution & Brand \\
\hline Mouse IgG1anti-rat CD8a & PE & OX-8 & $1: 200$ & BioLegend, USA \\
\hline Mouse IgG1 anti-rat RT1B & FITC & OX-6 & $1: 200$ & BD Biosciences, USA \\
\hline Mouse IgG1 anti-rat $\alpha \beta T C R$ & AF647 & R73 & $1: 200$ & BioLegend, USA \\
\hline Mouse IgG2a anti-rat CD11b/c & PE & OX-42 & $1: 200$ & BioLegend, USA \\
\hline Mouse IgG2a anti-rat CD11b/c & AF647 & OX-42 & $1: 200$ & BioLegend, USA \\
\hline Mouse IgG1 anti-rat CD45RA & PE & OX-22 & $1: 200$ & BioLegend, USA \\
\hline Mouse IgG1 anti-rat CD31 & PE & TLD-3A12 & $1: 100$ & BD Biosciences, USA \\
\hline Mouse IgG1 anti-rat CD4 & PE/Cy7 & W3/25 & $1: 200$ & BioLegend, USA \\
\hline Mouse IgG2a anti-rat CD172a & PE & OX-41 & $1: 100$ & BioLegend, USA \\
\hline Mouse IgG1 anti-rat CD45 & AF647 & OX-1 & $1: 100$ & BioLegend, USA \\
\hline Mouse IgM anti-rat RP3 & - & - & $1: 50$ & In-house production \\
\hline Rat IgG2a anti-mouse IgM & PE & R6-60.2 & $1: 200$ & BD Biosciences, USA \\
\hline Mouse IgG1 anti-rat CD25 & - & OX-39 & $1: 150$ & Bio-Rad, USA \\
\hline Mouse IgG1 Isotype control & - & - & $1: 150$ & Bio-Rad, USA \\
\hline Mouse IgG1 anti-rat TCR V $\beta 8.2 / 8.4$ & - & R78 & $1: 100$ & BioLegend, USA \\
\hline Mouse IgG1 anti-rat TCR V $\beta 8.5$ & - & B73 & $1: 100$ & Bio-Rad, USA \\
\hline Mouse IgG2a anti-rat TCR V $\beta 10$ & - & G101 & $1: 100$ & Bio-Rad, USA \\
\hline Mouse IgG2b anti-rat TCR V $\beta 16$ & - & HIS42 & $1: 100$ & OriGene, USA \\
\hline Goat anti-mouse IgG (H+L) & AF647 & Polyclonal & $1: 200$ & Jackson IR, USA \\
\hline
\end{tabular}




\subsubsection{Kits}

Phusion High-Fidelity DNA Polymerase Kit Thermo Fisher Scientific, USA

RevertAid First Strand cDNA Synthesis Kit Thermo Fisher Scientific, USA

MagSi-NGS Plus Kit

MagnaMedics, Netherlands

MiSeq Reagent Kit v3

Illumina, USA

QIAamp cador Pathogen Mini Kit

QIAGEN, Netherlands

QIAamp Fast DNA Stool Mini Kit

QIAGEN, Netherlands

Rat $\mathrm{CD} 4^{+} \mathrm{T}$ cell Isolation Kit

Stemcell Technologies, Canada

\subsubsection{Equipment and consumables}

\subsubsection{Equipment}

Cryobox

Metal cell strainer $(40 \mu \mathrm{m})$

MidiMACS Separator

Multichannel pipette

Pipettes $(2.5 ; 10 ; 20 ; 100 ; 200 ; 1000 \mu \mathrm{L})$

Pipetus

Small Animal Laryngoscope LS-2

Tuberculin glass syringes

Dounce homogenizer

\subsubsection{Consumables}

Cell strainers $(40 \mu \mathrm{m}, 70 \mu \mathrm{m})$

Winged catheter (18G)

Reaction tubes $(0.2,1.5,2 \mathrm{~mL})$

Conical centrifuge tubes $(15,50 \mathrm{~mL})$

Pipette tips $(10,100,200,1000 \mu \mathrm{L})$

Pipette filter tips $(10,100,200,1000 \mu \mathrm{L})$

Parafilm

Cell culture plates (12-, 24-, 96-well)

qPCR plates

Petri dishes $(5,10 \mathrm{~mL})$

Surgical suture

Syringes $(5,10 \mathrm{~mL})$

Cannulas (18G, 20G, 24G, 26G)

FACS tubes

MACS LD Columns
Nalgene, USA

UMG technical workshop, Germany

Miltenyi, Germany

StarLab, Gemany

Eppendorf, Gemany

Hirschmann, Gemany

Penn Century, USA

Poulten \& Graf, Germany

Kimble Chase, USA
Greiner Bio-One, Austria / Germany

B. Braun, Germany

Sarstedt, Germany

Greiner Bio-One, Austria / Germany

StarLab, Germany

StarLab, Germany

Pichiney Plastic Packaging, USA

Thermo Fisher Scientific, USA

StarLab, Germany

Greiner Bio-One, Germany

B. Braun, Germany

B. Braun, Germany

B. Braun, Germany

BD Biosciences, USA

Miltenyi, Germany 


\subsubsection{Instruments and machines}

\begin{tabular}{|c|c|}
\hline Axiovert 200M fluorescence microscope & Zeiss, Germany \\
\hline Axiovert $40 \mathrm{C}$ binocular microscope & Zeiss, Germany \\
\hline Centrifuge $5415 \mathrm{R}$ & Eppendorf, Gemany \\
\hline CytoFLEX S & Beckman Coulter, USA \\
\hline EV231 power supply & Consort, Belgium \\
\hline FACSAria II & BD Biosciences, USA \\
\hline FACSCalibur & BD Biosciences, USA \\
\hline \multirow[t]{2}{*}{ ImageQuant 100 image capture system } & GE Healthcare, USA \\
\hline & Amersham Biosciences, UK \\
\hline Heracell 240 incubator & Heraeus, Germany \\
\hline Laminar flow hood & Heraeus, Germany \\
\hline Mastercycler Nexus Gradient & Eppendorf, Germany \\
\hline Multifuge $1 \mathrm{~S}-\mathrm{R}$ & Heraeus, Germany \\
\hline NanoDrop-ND1000 & Thermo Fisher Scientific, US \\
\hline StepOnePlus Real-Time PCR System & Applied Biosciences, USA \\
\hline
\end{tabular}

\subsubsection{Software and plug-ins}

Ampvis2

BLAST+ (Version 2.7.1)

CellQuest Pro (Version c0.0)

cutadapt (Version 1.18)

FACSDiva Software (Version 8.0.1)

fastp (Version 0.19.4)

FlowJo (Version 10)

ggplot2

GraphPad Prism (Versions 6 - 8)

Microsoft Office $(2010,2016)$

MiSeq marker gene pipeline (Version 1.7)

PEAR (Version 0.9.11)

RStudio (Version 1.1.463)

StepOnePlus Software (Version 2.0)

VSEARCH (Version 2.9.1)

DAVID (Version 6.8)

$\mathrm{R}$ (Version 3.6.0)
Andersen et al., 2018

NCBI, USA

BD Biosciences, USA

Martin, 2011

BD Biosciences, USA

Chen et al., 2018

FlowJo LCC, USA

Wickham, 2016

GraphPad, USA

Microsoft, USA

Dominik Schneider

Zhang et al., 2014

RStudio, USA

Applied Biosciences, USA

Rognes et al., 2016

LHRI, USA

R Core Team 


\subsection{Methods}

\subsubsection{Animals}

6- 8 weeks old wild type and TCR ${ }^{\mathrm{MBP}}$-transgenic Lewis rats (Kitz, 2013) on a LEW/Crl (Rattus norvegicus) background were used for all experiments as well as the generation of $\mathrm{T}$ cell lines. The animals were kept in GR9000 IVC cages at a 12/12 h light/dark cycle with food and water provided ad libitum. The animals were bred and raised at the animal facilities of the University Medical Center Göttingen (UMG) in Göttingen and Holtensen. All experiments were performed in accordance with the local regulations of animal welfare of Lower Saxony, Germany.

\subsubsection{Generation and culture of primary $\mathrm{T}$ cell lines}

MBP- or OVA-reactive T cells retrovirally transduced to express eGFP (GFP) or mCherry (Cherry) were generated as reported previously (Flügel et al., 1999). 6- to 8-week-old female Lewis rats were briefly anesthetized with diethyl ether and immunized with an emulsion consisting to equal parts of guinea pig MBP or OVA (1 mg / mL) and CFA (2 mg / mL). The emulsion was prepared using tuberculin glass syringes (Poulten \& Graf, Germany) and injected subcutaneously at the tail base ( $50 \mu \mathrm{L}$ each side) and into the popliteal cavity ( $25 \mu \mathrm{L}$ each side). $9-10$ days p.i., the animals were asphyxiated with $\mathrm{CO}_{2}$. The draining lymph nodes (inguinal, paraaortic, popliteal) were isolated and brought to single cell suspension using a $40 \mu \mathrm{m}$ cell strainer. The cells were washed with $\mathrm{EH}$ and centrifuged at $1200 \mathrm{rpm}$ and $4{ }^{\circ} \mathrm{C}$ for $6 \mathrm{~min}$. Afterwards, the pellet was resuspended in RM and brought to a concentration of $4 \times 10^{6} \mathrm{lymph}$ node cells / $\mathrm{mL}$. The cells were subsequently co-cultured with GP+E86 packaging cells producing replication-deficient GFP or Cherry retroviruses carrying a G-418-resistance cassette and thereby transduced to express either fluorescent protein. To this end, the GP+E86 cells had previously been thawed and propagated in petri dishes in TCM containing $10 \%$ FCS and G-418 (Capricorn Scientific, Germany $0.4 \mathrm{mg} / \mathrm{mL}$ ) at $5 \% \mathrm{CO}_{2}$ and $37^{\circ} \mathrm{C}$ (Heracell 240 , Heraeus, Germany) for 7 days. When reaching confluency $3-4$ days prior to starting the T cell culture, the GP+E86 cells were trypsinated with 1x Trypsin-EDTA (Merck, Germany), diluted $1: 8-10$ with TCM containing $10 \%$ FCS, replated and incubated as described. $4-6 \mathrm{~h}$ prior to the lymph node cell isolation, the GP+E86 cells were again trypsinated, washed with $\mathrm{EH}$ and centrifuged at $1200 \mathrm{rpm}$ and $4{ }^{\circ} \mathrm{C}$ for $6 \mathrm{~min}$. The GP+E86 cell were resuspended in RM at a concentration of $3 \times 10^{5} / \mathrm{mL}$. $50 \mu \mathrm{L}$ of the GP+E86 cell suspension were equally distributed to the wells of U-bottom 96-well plates. The plates were incubated at $10 \% \mathrm{CO}_{2}$ and $37{ }^{\circ} \mathrm{C}$ (Heracell 240, Heraeus, Germany) until $50 \mu \mathrm{L}$ of the lymph node cell suspension were added to each well, resulting in $2 \times 10^{5}$ lymph node cells and $1.5 \times 10^{4} \mathrm{GP}+\mathrm{E} 86$ cells in a final volume of $100 \mu \mathrm{L}$ per well. In addition and dependent on the antigen used for immunization, MBP or OVA was added at a concentration of $7 \mu \mathrm{g} / \mathrm{mL}$ final volume. Incubation at $10 \% \mathrm{CO}_{2}$ and 37 ${ }^{\circ} \mathrm{C}$ was continued. 2 days later, $50 \mu \mathrm{L}$ TCGF were added to each well. Another 2 days later, 100 $\mu \mathrm{L}$ supernatant were gently removed and substituted with TCGF containing G-418 at $0.4 \mathrm{mg}$ / 
$\mathrm{mL}$ final volume. Additionally, the cells were transferred into flat bottom 96-well plates. On D7 after initial stimulation, the MBP- or OVA-specific T cells were re-stimulated: $100 \mu \mathrm{L}$ supernatant were discarded and substituted with $100 \mu \mathrm{L}$ RM containing $1.4 \times 10^{6}$ irradiated thymocytes, $7 \mu \mathrm{g} / \mathrm{mL}$ MBP or OVA and $0.4 \mathrm{mg} / \mathrm{mL}$ G-418. 2 days later, $50 \mu \mathrm{L}$ TCGF with 0.4 $\mathrm{mg} / \mathrm{mL}$ G-418 were added to each well. Retroviral transduction efficiency was tested 1 or 2 days later using an Axiovert 200M fluorescence microscope (Zeiss, Germany). Cell suspensions of $30-50$ wells / plate containing T cells of bright fluorescence indicative of high transduction efficiency were pooled into small $(5 \mathrm{~mL}$ ) Petri dishes (Greiner Bio-One, Germany) and $1.5 \mathrm{~mL}$ TCGF were added. On D7 post-stimulation, $\mathrm{GFP}^{+} \mathrm{T}$ cells were re-stimulated by co-culturing 3.5 $\times 10^{6} \mathrm{~T}$ cells with $70 \times 10^{6}$ irradiated thymocytes in $5 \mathrm{~mL} \mathrm{RM}$ with $7 \mu \mathrm{g} / \mathrm{mL}$ antigen and 0.4 $\mathrm{mg} / \mathrm{mL}$ G-418. T cell proliferation was boosted 2 days later by adding TCGF and G-418. Upon reaching a certain cell density, the cell suspensions were diluted $2: 3$ or $3: 4$ with TCGF and G-418 and transferred into large $(10 \mathrm{~cm})$ cell culture dishes. This procedure was repeated until the cells started to become resting around D4 post-stimulation. In total, this 7-day T cell restimulation rhythm could be repeated up to 4 times. From the $3^{\text {rd }}$ re-stimulation on, $T$ cells could be frozen. To this end, $20-50 \times 10^{6} \mathrm{~T}$ cell blasts (D2 after stimulation) or resting $\mathrm{T}$ cells (D6 after stimulation) were centrifuged at $1200 \mathrm{rpm}$ and $4{ }^{\circ} \mathrm{C}$ for $6 \mathrm{~min}$. The pellet was resuspended in $1.5 \mathrm{mLFM}$, transferred into freezing vials and slowly cooled down to $-80{ }^{\circ} \mathrm{C}$ in cryoboxes (Nalgene, USA) during the course of $24 \mathrm{~h}$ before being transferred to liquid nitrogen. Whenever needed, the cells could be quickly thawed in $10 \mathrm{~mL} \mathrm{TCM}+10 \% \mathrm{FCS}$, washed in $\mathrm{EH}$ and centrifuged at $1200 \mathrm{rpm}$ and $4^{\circ} \mathrm{C}$ for $6 \mathrm{~min}$. Subsequently, they were either adoptively transferred into recipient rats or further propagated as described.

$T$ cell lines from $T_{C R}{ }^{M B P}$-transgenic rats were generated and cultured as described above with the following exceptions: draining lymph nodes were isolated and processed $5-6$ instead of 9 - 10 days p.i.; T cell re-stimulation cycles were shortened to 6 instead of 7 days; and the antigen concentration was $5 \mu \mathrm{g} / \mathrm{mL}$.

\subsubsection{EAE induction}

\subsubsection{Adoptive T cell transfer}

Resting effector memory T cells (D6 after stimulation) specific for MBP or OVA (TMBP-EM / TovAEM cells) or fully activated T cell blasts (D2 after stimulation) specific for MBP (or TMBP-blast cells) were resuspended in $\mathrm{EH}$ and brought to the desired concentration. If not stated otherwise, $7.5 \times 10^{6}$ resting TMBP-EM or TOVA-EM cells, or $2.5 \times 10^{5} \mathrm{~T}_{\text {MBP-blast }}$ cells per animal were transferred i.v. in rats briefly anesthetized with diethyl ether.

\subsubsection{Intratracheal immunization}

6- to 10-week-old rats that had previously received $7.5 \times 10^{6} \mathrm{~T}_{\text {MBP-EM }}$ cells i.v. were briefly anesthetized with diethyl ether and immunized i.tr. with an emulsion consisting to equal parts 
of guinea pig MBP (varying doses, as indicated in the results section) or OVA (0.02 $\mathrm{mg} / \mathrm{mL}$ ) and CFA (varying doses, as indicated in the results section). The emulsion was prepared as described above (see 2.2.2). The rats were fixed (with a bar behind their upper incisors) on a stand in an upright position slightly leaning backwards. The trachea was located using a Small Animal Laryngoscope LS-2 (Penn Century, USA). Each animal received a total volume (in $\mu \mathrm{L}$ ) corresponding to around one third of its body weight (in g), e.g. $100 \mu \mathrm{L}$ emulsion per $300 \mathrm{~g}$ body weight. The emulsion was instilled into the trachea through a winged $18 \mathrm{G}$ catheter (B. Braun, Germany). The animals were released from the stand and transferred to their cages to recover. The entire procedure typically lasted approximately $1 \mathrm{~min}$ per animal.

\subsubsection{Subcutaneous immunization}

6- to 10-week-old rats that had previously received $7.5 \times 10^{6} \mathrm{~T}_{\text {MBP-EM }}$ cells i.v. were briefly anesthetized with diethyl ether and immunized with an emulsion consisting to equal parts of guinea pig MBP or OVA (1 mg / mL) and CFA ( $1 \mathrm{mg} / \mathrm{mL}$ ). The emulsion was prepared as described (see 2.2.2) and injected subcutaneously into the popliteal cavities of both hind limbs. In total, each animal received a volume (in $\mu \mathrm{L}$ ) corresponding to around one-third of its body weight (in g), e.g. $100 \mu \mathrm{L}$ emulsion per $300 \mathrm{~g}$ body weight. Subsequently, the animals were returned to their cages to recover. The entire procedure typically lasted approximately 1 min per animal.

\subsubsection{EAE scoring system}

After EAE induction, animals were weighed and their clinical symptoms assessed daily as follows:

$\begin{array}{cl}\text { Score } & \text { Symptoms } \\ 0 & \text { No clinical symptoms } \\ 1 & \text { Tail paralysis } \\ 2 & \text { Ataxia } \\ 3 & \text { Hind limb paralysis } \\ 4 & \text { Tetraparesis } \\ 5 & \text { Moribund }\end{array}$

Animals showing signs of forelimb paralysis (score $>3$ ) were sacrificed. 


\subsubsection{Organ retrieval and processing}

\subsubsection{Preparation of single cell suspensions from different organs}

Animals were sacrificed by $\mathrm{CO}_{2}$ asphyxiation. Organs of interest were retrieved, weighed and subsequently brought to single cell suspension as described below. If not stated otherwise, all organs were kept on ice in $\mathrm{EH}$ until processed.

Blood

Blood was retrieved from the heart by cardiac puncture with syringes prefilled with $0.2 \mathrm{~mL}$ of $\mathrm{Na}_{2}$ XEDTA (80 mmol / L; Roth, Germany) and mixed 1:1 with 1x PBS (RT). The samples were underlayed with $3 \mathrm{~mL}$ of LSM1077 (PromoCell, Germany) and centrifuged for $30 \mathrm{~min}$ at 2000 rpm and RT with minimum acceleration and brake settings. The lymphocyte-enriched interphase was aspired, washed with $\mathrm{EH}$ and centrifuged at $1200 \mathrm{rpm}$ and $4{ }^{\circ} \mathrm{C}$ for $6 \mathrm{~min}$ (standard washing procedure). The pellets were resuspended in $1 \mathrm{~mL} \mathrm{ACK}$ buffer and incubated for 4 minutes on ice for erythrolysis. The samples were washed again with $\mathrm{EH}$ and taken up in $1 \mathrm{~mL}$ EH $+2 \mathrm{mM} \mathrm{Na}$ xEDTA and passed through $70 \mu \mathrm{m}$ filter caps into FACS tubes. Lung

Lungs were thoroughly and repeatedly sectioned using a tissue chopper (Mcllwain, UK). The homogenized tissue was washed with $\mathrm{EH}$ as described above. Pellets were resuspended and incubated with $2 \mathrm{~mL}$ of $0.3 \%$ collagenase in $1 \times$ PBS for $30 \mathrm{~min}$ at $37^{\circ} \mathrm{C}$ under constant shaking. Subsequently, the tissue was forced through a cell strainer $(40 \mu \mathrm{m})$ and washed with $\mathrm{EH}$ as described above. The pellet was resuspended in $5 \mathrm{~mL} 40 \%$ percoll and underlayed with $5 \mathrm{~mL}$ $70 \%$ percoll. After 30 min of centrifugation at $2000 \mathrm{rpm}$ and $4{ }^{\circ} \mathrm{C}$ with minimum acceleration and brake settings, the leukocyte-enriched interphase was aspired and washed with $\mathrm{EH}$ as described. Finally, the pellets were resuspended in $1 \mathrm{~mL} \mathrm{EH}+2 \mathrm{mM} \mathrm{Na}{ }_{2}$ XEDTA and passed through $70 \mu \mathrm{m}$ filter caps into FACS tubes.

Lymph nodes

Lymph nodes were passed through a cell strainer $(40 \mu \mathrm{m})$, washed with $\mathrm{EH}$ and taken up in 1 $\mathrm{mL} E \mathrm{EH}+2 \mathrm{mM} \mathrm{Na} 2 \mathrm{xEDTA}$ and passed through $70 \mu \mathrm{m}$ filter caps into FACS tubes.

Spleen

Spleens were passed through a cell strainer $(40 \mu \mathrm{m})$, washed with $\mathrm{EH}$, resuspended in $5 \mathrm{~mL}$ ACK buffer and incubated for 4 minutes on ice for erythrolysis. The samples were washed again with $\mathrm{EH}$ and taken up in $5 \mathrm{~mL} \mathrm{EH}+2 \mathrm{mM} \mathrm{Na}{ }_{2}$ XEDTA. One $\mathrm{mL}$ of the final suspension was passed through $70 \mu \mathrm{m}$ filter caps into a FACS tube.

Spinal cord

Spinal cords were passed through a cell strainer $(40 \mu \mathrm{m})$ and taken up in $15 \mathrm{~mL}$ of EH. Cell suspensions were mixed with $5 \mathrm{~mL}$ isopercoll, underlayed with $5 \mathrm{~mL}$ underlay percoll and centrifuged for $30 \mathrm{~min}$ at $2780 \mathrm{rpm}$ and $4{ }^{\circ} \mathrm{C}$. The leukocyte-enriched interphase was aspired and washed with EH. Finally, the pellets were resuspended in $1 \mathrm{mLEH}+2 \mathrm{mM} \mathrm{Na} \mathrm{a}_{2} \mathrm{EDTTA}$ and passed through $70 \mu \mathrm{m}$ filter caps into FACS tubes. 


\subsubsection{Isolation of antigen-presenting cells from the lung to stimulate $T_{M B P-E M}$ cells}

Lung were isolated, sectioned, digested with $0.3 \%$ collagenase and passed through a cell strainer $(40 \mu \mathrm{m})$ as described above (see 2.2.4.1) with the exception that APC isolation buffer was used for all washing steps instead of EH. Subsequently, the samples were centrifuged at $1200 \mathrm{rpm}$ and $4{ }^{\circ} \mathrm{C}$ for $6 \mathrm{~min}$. The supernatant was aspired and the pellet resuspended in 10 $\mathrm{mL} \mathrm{APC}$ isolation buffer and underlayed with $3 \mathrm{~mL}$ of $14.5 \%$ Nycodenz solution. Gradient centrifugation was performed for $25 \mathrm{~min}$ at $450 \times \mathrm{g}$ and RT. The interphase was collected, washed with APC isolation buffer and centrifuged at $1200 \mathrm{rpm}$ and $4{ }^{\circ} \mathrm{C}$ for $6 \mathrm{~min}$. The pellet was resuspended in $1 \mathrm{~mL}$ RM. Subsequently, the composition of the isolated cell fraction was determined using antibody labeling and flow cytometry (see 2.2.8.1 and 2.2.8.2). In addition, the isolated cells were tested for their capacity to activate T cells. $5 \times 10^{4}$ GFP $^{+} \mathrm{T}_{\text {MBP-EM }}$ cells and $1 \times 10^{5}$ isolated lung cells were co-incubated in $100 \mu \mathrm{L}$ RM with $10 \mu \mathrm{g} / \mathrm{mL} \mathrm{MBP}$ in flat bottom 96-well plates. Two days later, $2.5 \times 10^{5} \mathrm{~T}_{\text {MBP-GFP }}$ cells were adoptively transferred into naïve recipient rats and the clinical course of EAE was monitored over the following days.

\subsubsection{Sorting of endothelial cells from spinal cord parenchyma and meninges}

Spinal cord parenchyma was brought to single cell suspension using a Dounce homogenizer and taken up in $50 \mathrm{~mL} \mathrm{EH}$. Meninges were manually cut into small pieces and taken up in 15 $\mathrm{mL} \mathrm{EH}$. The samples were centrifuged at $1200 \mathrm{rpm}$ and $4{ }^{\circ} \mathrm{C}$ for $6 \mathrm{~min}$. The supernatant was aspired and the parenchyma and meninges pellets taken up in 1 and $0.5 \mathrm{~mL}$ digestion buffer, respectively. The samples were incubated at $37{ }^{\circ} \mathrm{C}$ for $1 \mathrm{~h}$ and resuspended every $10 \mathrm{~min}$. Subsequently, the reaction was stopped by adding $15 \mathrm{~mL}$ of $\mathrm{EH}+2 \mathrm{mM} \mathrm{Na} \mathrm{a}_{2} \mathrm{EDTT}$. The cell suspensions were passed through $70 \mu \mathrm{m}$ cell strainers and mixed with $5 \mathrm{~mL}$ isopercoll. The samples were centrifuged for $30 \mathrm{~min}$ at $2780 \mathrm{rpm}$ and $4{ }^{\circ} \mathrm{C}$. The supernatant was discarded and the pellets resuspended in $100 \mu \mathrm{L} 1 \mathrm{x}$ PBS. Subsequently, the endothelial cells were antibody-labelled and sorted using fluorescence-activated cell sorting (see 2.2.8.1 and 2.2.8.3). The sorted cells were centrifuged at $4000 \mathrm{rpm}$ and $4{ }^{\circ} \mathrm{C}$ for $4 \mathrm{~min}$ and the pellet was resuspended in $200 \mu \mathrm{L}$ TRI reagent (Merck, Germany) and $1 \mu \mathrm{L}$ glycogen (Roche, Switzerland). The samples were stored at $-80^{\circ} \mathrm{C}$ until further processed. Gene expression was determined using qPCR (see 2.2.9).

\subsubsection{Re-transfer of $T_{M B P}$ cells from spleen}

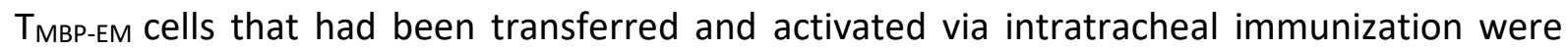
isolated from donor animals 3.5 days p.i and transferred into naïve recipient rats. Briefly, the spleens were retrieved and prepared as described above (see 2.2.4.1). After the erythrolysis, the cells suspension of each spleen was taken up in $10 \mathrm{~mL} \mathrm{TCM}$ and incubated in petri dishes for $30 \mathrm{~min}$ at $37^{\circ} \mathrm{C}$ and $10 \% \mathrm{CO}_{2}$ allowing the adhesion of macrophages to the plates. This way, the cell suspension was enriched for non-adherent cells such as lymphocytes. The 
resulting cell suspension was subsequently washed in $\mathrm{EH}$ and centrifuged at $1200 \mathrm{rpm}$ and 4 ${ }^{\circ} \mathrm{C}$ for $6 \mathrm{~min}$. The pellet was taken up in $1 \mathrm{~mL}$ EH and passed through $70 \mu \mathrm{m}$ filter caps into FACS tubes. The suspensions of each group were pooled and the number of contained GFP ${ }^{+}$ $T_{M B P}$ cells determined using flow cytometry (see 2.2.8.2). Subsequently, the cell suspensions of each group were brought to a concentration of $2.5 \times 10^{6} \mathrm{GFP}^{+} \mathrm{T}_{\mathrm{MBP}}$ cells / $\mathrm{mL}$ with EH. Finally, $1 \mathrm{~mL}$ of suspension per animal was injected i.v. into naïve recipients.

\subsubsection{Lung and gut microbiota identification}

\subsubsection{Intratracheal administration of neomycin}

Rats were briefly anesthetized and intubated as described above (see 2.2.3.2). A total volume of $150 \mu \mathrm{L}$ neomycin (0.1, 1 or $5 \mathrm{mg}$; Thermo Fisher Scientific, USA) dissolved in sterile 1x PBS or sterile $1 \times$ PBS alone was instilled into the trachea through a winged $18 \mathrm{G}$ catheter (B. Braun, Germany). The animals were released from the stand and transferred to their cages to recover. The entire procedure typically lasted approximately 1 min per animal.

\subsubsection{Bronchoalveolar lavage}

Bronchoalveolar lavage (BAL) was performed under sterile conditions on animals that had been treated with neomycin of varying concentrations or sterile $1 x$ PBS for 7 days. Briefly, the rats were sacrificed using $\mathrm{CO}_{2}$. Under the laminar flow hood and after carefully disinfecting the animal's fur with $70 \% \mathrm{EtOH}$, a tracheotomy was performed and a sterile gavage needle inserted into the trachea. The gavage needle was fixed and held in position using surgical suture (B. Braun, Germany). $5 \mathrm{~mL}$ pre-warmed $\left(37^{\circ} \mathrm{C}\right) 1 \times$ PBS were slowly instilled into the lung and after $30 \mathrm{~s}$ a volume of $4 \mathrm{~mL}$ was retrieved. This step was repeated once, yielding 8 $\mathrm{mL}$ of total BAL fluid per animals. The samples were snap-frozen on dry ice in $2 \mathrm{~mL}$ aliquots and stored at $-80^{\circ} \mathrm{C}$ until further processed.

\subsubsection{DNA isolation from bronchoalveolar lavage (BAL) fluid}

To efficiently extract DNA from both Gram-positive and Gram-negative bacteria, a pre-lysis step was performed by incubating $400 \mu \mathrm{L}$ BAL fluid with $2 \mu \mathrm{L}$ fresh chicken egg lysozyme (100 $\mathrm{mg} / \mathrm{mL}$; Serva, Germany) at $37^{\circ} \mathrm{C}$ and $180 \mathrm{rpm}$ for $1 \mathrm{~h}$.

Subsequently, DNA was isolated using the QIAamp cador Pathogen Mini Kit (QIAGEN, Netherlands) according to the manufacturer's instructions. In the final step, isolated DNA was eluted in $50 \mu \mathrm{L}$ sterile $\mathrm{H}_{2} \mathrm{O}$. 


\subsubsection{DNA isolation from fecal samples}

To homogenize the fecal samples, about $150 \mathrm{mg}$ of material was incubated in $1 \mathrm{~mL}$ InhibitEX buffer (QIAGEN, Netherlands) at $37{ }^{\circ} \mathrm{C}$ for $15 \mathrm{~min}$. The samples were then homogenized using an inoculation loop and incubated at $37^{\circ} \mathrm{C}$ for another $5 \mathrm{~min}$. Subsequently, DNA was isolated using the QIAamp Fast DNA Stool Mini Kit (Netherlands, Germany) according to the manufacturer's instructions. In the final step, isolated DNA was eluted in $200 \mu \mathrm{L}$ sterile $\mathrm{H}_{2} \mathrm{O}$.

\subsubsection{BAL fluid and fecal sample DNA quantification}

The isolated DNA was quantified and its purity determined based on the $260 / 280$ ratio using the NanoDrop-ND1000 (Thermo Fisher Scientific, USA). The Nanodrop was first cleaned using $5 \mu \mathrm{L}$ and calibrated using $2 \mu \mathrm{L}$ of sterile $\mathrm{H}_{2} \mathrm{O}$. Subsequently, $2 \mu \mathrm{L}$ of each sample were measured.

\subsubsection{Gel electrophoresis}

Extraction of metagenomic DNA and amplicon production were verified via gel electrophoresis. Agarose gel was prepared by boiling $0.8 \%$ low-electroendosmosis (LE)Agarose (Biozym, Germany) in 1x TAE. The agarose was filled into a gel electrophoresis chamber which was filled with $1 \times$ TAE after polymerization of the gel. The pockets of the gel were loaded with $4 \mu \mathrm{L}$ DNA sample and $2 \mu \mathrm{L} 6 \times$ Orange DNA Loading Dye (Thermo Fisher Scientific, USA). $2 \mu \mathrm{L}$ of GeneRuler $1 \mathrm{~kb}$ DNA ladder (Thermo Scientific, USA) flanking the DNA samples were used as size reference. Electrophoresis was performed at $100 \mathrm{mV}$ for $50 \mathrm{~min}$. The gel was subsequently stained in an ethidium bromide bath $(0.5 \mu \mathrm{g} / \mathrm{mL})$ for $10 \mathrm{~min}$ allowing the ethidium bromide to intercalate between the DNA base pairs. Afterwards, the stained DNA molecules were visualized with ultraviolet light (302 nm) using the ImageQuant 100 image capture system (GE Healthcare, USA / Amersham Biosciences, UK).

\subsubsection{PCR amplification of bacterial amplicons}

The 16S rRNA region is highly conserved among bacteria and thus allows for the determination of bacterial strains and phylogeny (Janda and Abbott, 2007). Bacterial 16S rRNA amplicons were amplified via PCR using the bacterial primers targeting the V3 - V4 region described by Klindworth and colleagues (2013) including adapters for Illumina MiSeq sequencing: 
Table 2: Primer-sequences of 165 rRNA V3 - V4 region

\begin{tabular}{|l|l|}
\hline Primer (16S rRNA region) & Primer sequence (5' -3') $^{\prime}$ \\
\hline MiSeq_B_V3_for_klindworth & $\begin{array}{l}\text { TCGTCGGCAGCGTCAGATGTGTATAAGAGACAGCCTACGGG } \\
\text { NGGCWGCAG }\end{array}$ \\
\hline MiSeq_B_V4_rev_klindworth & $\begin{array}{l}\text { GTCTCGTGGGCTCGGAGATGTGTATAAGAGACAGGACTACH } \\
\text { VGGGTATCTAATCC }\end{array}$ \\
\hline \multicolumn{2}{|l|}{ Bold = binding sequence / cursive = MiSeq overhang } \\
\hline
\end{tabular}

For each PCR reaction, a master mix was prepared as follows:

Table 3: Master mix for amplicon PCR

\begin{tabular}{|l|l|}
\hline Reagent & Volume $(\boldsymbol{\mu L})$ / reaction \\
\hline $5 x$ Phusion GC Buffer & 10 \\
\hline 10 mM dNTPs & 1 \\
\hline DMSO & 2.5 \\
\hline MgCl2 & 0.2 \\
\hline MiSeq_B_V3_for_klindworth primer $(10 \mu \mathrm{M})$ & 1 \\
\hline MiSeq_B_V4_rev_klindworth primer $(10 \mu \mathrm{M})$ & 1 \\
\hline Phusion polymerase & 0.5 \\
\hline Template DNA & $7 \mu \mathrm{L}$ BAL fluid / $6 \mu \mathrm{L}$ feces \\
\hline Nuclease-free $\mathrm{H}_{2} \mathrm{O}$ & ad $50 \mu \mathrm{L}$ \\
\hline
\end{tabular}

For each reaction, $43 \mu \mathrm{L}$ master mix and $7 \mu \mathrm{L}$ or $6 \mu \mathrm{L}$ template DNA (100 - $150 \mathrm{ng}$ ) for BAL fluid and feces, respectively, were gently mixed in cooled PCR reaction tubes. For the negative control $7 \mu \mathrm{L}$ nuclease-free $\mathrm{H}_{2} \mathrm{O}$ and for the positive control $7 \mu \mathrm{L}$ of $B$. thuringiensis BTI18246 DNA (diluted 1:7 with nuclease-free $\mathrm{H}_{2} \mathrm{O}$ ) were used instead of DNA isolated from BAL fluid or feces. The reaction tubes were placed in a Mastercycler Nexus Gradient thermo cycler (Eppendorf, Germany). The PCRs were performed in triplicates with the following cycler settings (Table 4):

Table 4: PCR cycler settings

\begin{tabular}{|l|l|l|l|}
\hline Step & Temperature $\left({ }^{\circ} \mathrm{C}\right)$ & Time $(\mathbf{s})$ & Cycles \\
\hline Initial denaturation & 98 & 60 & $1 x$ \\
\hline Denaturation & 98 & 45 & \multirow{2}{*}{$25 x$ (feces) / } \\
\hline Annealing & 62 & 45 & $35 x$ (BAL fluid) \\
\hline Elongation & 72 & 30 (feces) / 45 (BAL fluid) & $1 x$ \\
\hline Final elongation & 72 & 300 & \\
\hline Hold & 4 & $\infty$ & \\
\hline
\end{tabular}

The amplicon synthesis was subsequently tested using $0.8 \%$ gel electrophoresis as described above (see 2.2.6.6). 


\subsubsection{Purification of amplicon DNA}

The amplified DNA was purified to remove unwanted side products and reagents using the MagSi-NGS Plus Kit (MagnaMedics, Netherlands) according to the manufacturer's instructions. After purification, amplicon DNA from BAL fluid and feces samples was eluted in $40 \mu \mathrm{L}$ sterile $\mathrm{H}_{2} \mathrm{O}$. The final concentration was determined using the NanoDrop as described above (see 2.2.6.5).

\subsubsection{Bacterial amplicon sequencing, data processing and data analysis}

Bacterial amplicon sequencing was performed at the Department of Genomic and Applied Microbiology and the Göttingen Genomics Laboratory of the University Göttingen under supervision of Prof. Dr. Rolf Daniel. Indexing of the PCR products was performed using the Nextera XT Index kit according to the manufacturer's instructions (Illumina, San Diego, CA, USA). Sequencing of $16 S$ rRNA amplicons was performed via the dual index paired-end approach ( $2 \times 300$ bp) with v3 chemistry for the Illumina MiSeq platform.

Processing of raw data and data analysis was performed by Dr. Jacqueline Hollensteiner. The MiSeq marker gene pipeline designed by Dr. Dominik Schneider was applied. Briefly, raw paired-end reads were quality-filtered using fastp (Chen et al., 2018). PEAR was used for read merging (Zhang et al., 2014). Subsequently, primer clipping was performed using cutadapt (Martin, 2011). Reads shorter than $300 \mathrm{bp}$ and chimeras were filtered out using VSEARCH (Rognes et al., 2016). The abundance table was based on exact amplicon sequence variants (ASV) with $100 \%$ similarity. The sequences were analysed via BLAST+ (NCBI, USA). The resulting taxonomy was entered into the abundance table. Subsampling of ASV tables, diversity analysis and graphical depiction of data was performed using RStudio (RStudio, USA) and the R packages ampvis2 (Andersen et al., 2018) and ggplot2 (Wickham, 2011). Alphadiversity metrics were performed in order to characterize bacterial diversity within each sample. These alpha-diversity metrics included the Shannon, Simpson, Invsimpson and Chao1 indices and were plotted in Graphpad Prism (V8; GraphPad, USA).

\subsection{7 $\mathrm{T}_{\text {MBP-EM }}$ cell activation and proliferation assay}

The activation and proliferation potential of $\mathrm{T}_{\mathrm{MBP}-\mathrm{EM}}$ cells was determined in two different experimental setups:

$T_{M B P-E M}$ cell anergy assay

$T_{\text {MBP }}$ cells from mediastinal lymph nodes of animals that had previously been transferred with

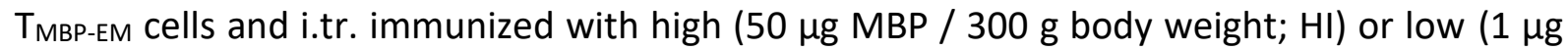
MBP / 300 g body weight; LO-OPT) concentrations of MBP were isolated and purified by negative selection for $\mathrm{CD}^{+}{ }^{+} \mathrm{T}$ cells (Stemcell Technologies, Canada) on D5 p.i.

$5 \times 10^{4}$ isolated TMBP cells and $5 \times 10^{5}$ irradiated thymocytes in $100 \mu \mathrm{L}$ RM with $10 \mathrm{mg} / \mathrm{mL}$ (+MBP), or without antigen (-MBP) were distributed to the wells of 96-well flat bottom plates (Thermo Fisher Scientific, USA). To determine T cell activation on transcriptional level, the cell 
suspensions of the corresponding wells were centrifuged at $4000 \mathrm{rpm}$ and $4{ }^{\circ} \mathrm{C}$ for $4 \mathrm{~min}$ and resuspended in $300 \mu \mathrm{L}$ TRI reagent (Merck, Germany) and $1 \mu \mathrm{L}$ glycogen (Roche, Switzerland) $12 \mathrm{~h}$ after in vitro stimulation. The samples were stored at $-80^{\circ} \mathrm{C}$ until further processed. Activation on mRNA level was quantified based on the expression of IFN $\gamma$ and IL-17 using qPCR (see 2.2.9).

To determine $T$ cell activation on protein level, the cells were stained for surface expression of CD25 as described below (see 2.2.8.1) and acquired using flow cytometry (see 2.2.8.2) $48 \mathrm{~h}$ after in vitro stimulation. T cell proliferation was quantified by flow cytometry on D2, 3 and 4 after in vitro stimulation by adding a defined number of Calibrite APC beads (BD Biosciences, USA) to the samples. On D2, $50 \mu \mathrm{L}$ TCGF were added to wells measured on D3 (and 4). On D3, another $50 \mu \mathrm{L}$ TCGF were added to the wells acquired on D4.

Neomycin toxicity assay

$5 \times 10^{4} \mathrm{~T}_{\mathrm{MBP}-\mathrm{EM}}$ cells and $5 \times 10^{5}$ irradiated thymocytes in $100 \mu \mathrm{L} \mathrm{RM}$ with $10 \mathrm{mg} / \mathrm{mL}$ were distributed to the wells of 96 -well flat bottom plates (Thermo Fisher Scientific, USA). Neomycin was added in various concentration (100 $\mathrm{ng}-100 \mathrm{mg} / \mathrm{mL}$ ) to test a potential cytotoxic effect on $\mathrm{T}$ cells. The cells were incubated at $37{ }^{\circ} \mathrm{C}$ and $10 \% \mathrm{CO}_{2}$.

$T$ cell proliferation was quantified by flow cytometry on D2, 3 and 4 after in vitro stimulation by adding a defined number of Calibrite APC beads (BD Biosciences, USA) to the samples. On $\mathrm{D} 2,50 \mu \mathrm{L}$ TCGF were added to wells measured on D3 (and 4). On D3, another $50 \mu \mathrm{L}$ TCGF were added to the wells acquired on D4.

Furthermore, $2.5 \times 10^{5} \mathrm{~T}_{\text {MBP-blast }}$ cells (D2 after stimulation) were adoptively transferred into naïve recipient rats and the clinical course of EAE was monitored over the following days.

\subsubsection{Flow cytometry}

\subsubsection{Cell staining}

Single cell suspensions from organs were prepared as described above (see 2.2.4). To stain immune cell populations, $100 \mu \mathrm{L}$ single cell suspension were transferred to a conical bottom 96-well plate (Thermo Fisher Scientific, USA). The plate was centrifuged for $3 \mathrm{~min}$ at $4{ }^{\circ} \mathrm{C}$ and $1300 \mathrm{rpm}$. The cells were washed with 1x PBS, centrifuged again and blocked with FACS buffer on ice for $3 \mathrm{~min}$. The plate was again centrifuged and the cells resuspended in primary antibody mixes diluted in FACS buffer (antibodies and dilutions listed under 2.1.4). The samples were incubated on ice and protected from light for 30 minutes. In case a secondary antibody staining was performed, the washing, blocking and staining steps were repeated. Finally, the cells were centrifuged and taken up in $100 \mu \mathrm{L}$ FACS buffer. The samples were acquired using flow cytometry. 


\subsubsection{Flow cytometry cell quantification}

A defined number of Calibrite APC beads (BD Biosciences, USA) was added to $100 \mu \mathrm{L}$ cell suspension from culture or from organs prepared as described (see 2.2.4) containing fluorescent $T_{M B P}$ cells or antibody-stained immune cell populations (Fig. 5). The samples were acquired using a FACSCalibur (BD Biosciences, USA) or a CytoFLEX S (Beckman Coulter, USA). Data analysis was performed using FlowJo (BD Biosciences, USA). In order to quantify a cell population, cell number and number of added beads were correlated and normalized to sample volume or organ weight if applicable.

Fig. 5 shows the exemplified gating strategies used for the flow cytometry acquisition of different immune cell populations.

\subsubsection{Flow cytometry cell sorting}

Single cell suspensions from organs were prepared as described (see 2.2.4). GFP ${ }^{+} \mathrm{T}_{\mathrm{MBP}}$ cells (from lung, mediastinal lymph nodes, blood and spinal cord) or endothelial cells (from spinal cord meninges or parenchyma) were sorted using a FACSAria III (BD Biosciences, USA) at a low flow rate. The cells were collected in EH-prefilled collection tubes under constant cooling (4 $\left.{ }^{\circ} \mathrm{C}\right)$. Typically, in order to perform qPCR, $300-5000$ T cells or $300-3000$ endothelial cells were sorted.

In case T cells were sorted for next-generation sequencing (NGS), the single cell suspensions of 3-4 animals were pooled and $C D 11 b^{+}$cells were depleted prior to sorting using Anti-PE MicroBeads for MACS separation (Miltenyi Biotech, Germany) according to the manufacturer's instructions. This way the samples were enriched for lymphocytes and a total of 20,000 cells was sorted. Finally, the sorted cells were centrifuged at $4000 \mathrm{rpm}$ and $4{ }^{\circ} \mathrm{C}$ for $4 \mathrm{~min}$ and the pellet was resuspended in $300 \mu \mathrm{L}$ TRI reagent (Merck, Germany) and $1 \mu \mathrm{L}$ glycogen (Roche, Switzerland). The samples were stored at $-80{ }^{\circ} \mathrm{C}$ until further processed. Gene expression was determined using qPCR (see 2.2.9).

Fig. 6 shows the exemplified gating strategies used for the cell sorting of T cells or endothelial cells. 
A General Gating Strategy

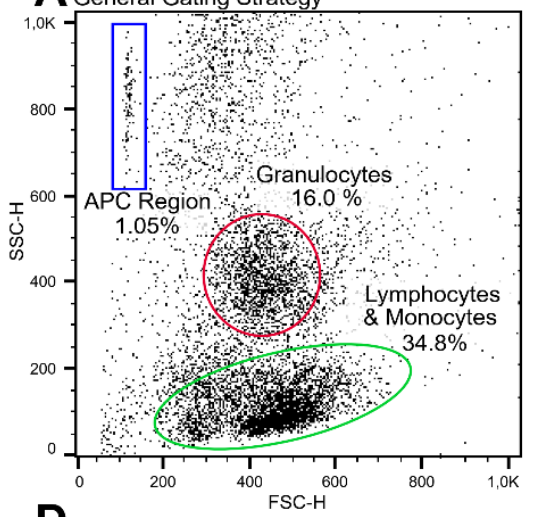

Dated on Lymphocytes \& Monocytes

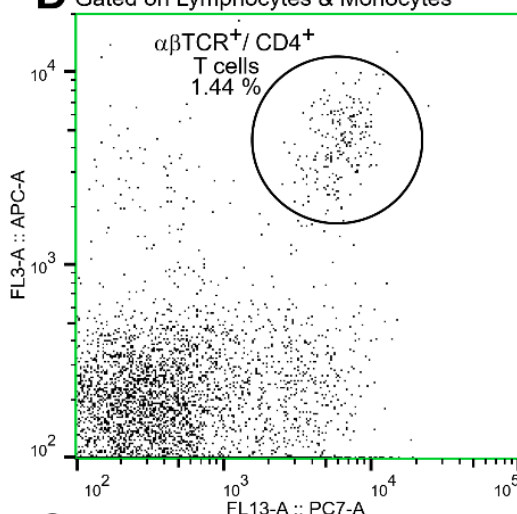

G Gated on Lymphocytes \& Monocytes

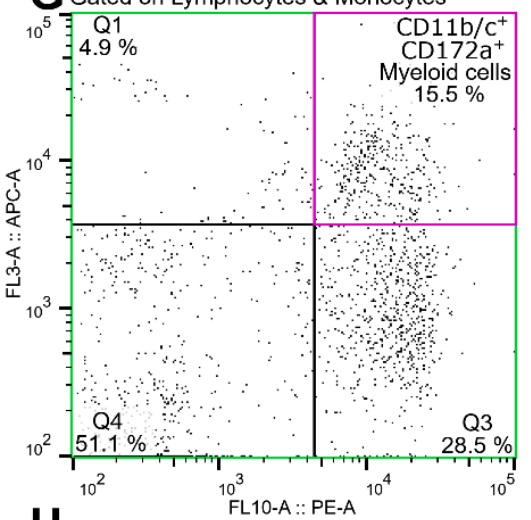

$\mathrm{H}_{\text {Gated on Granulocytes }}$

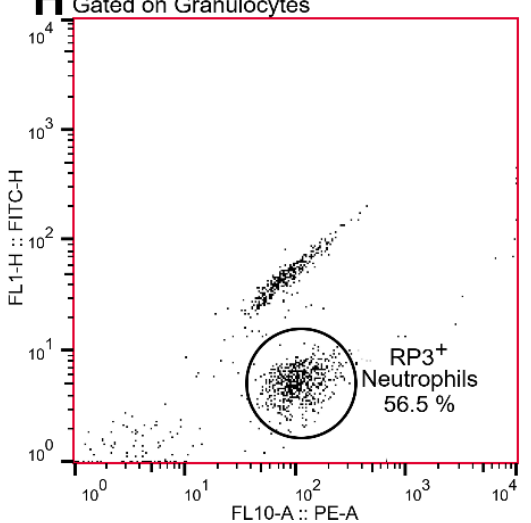

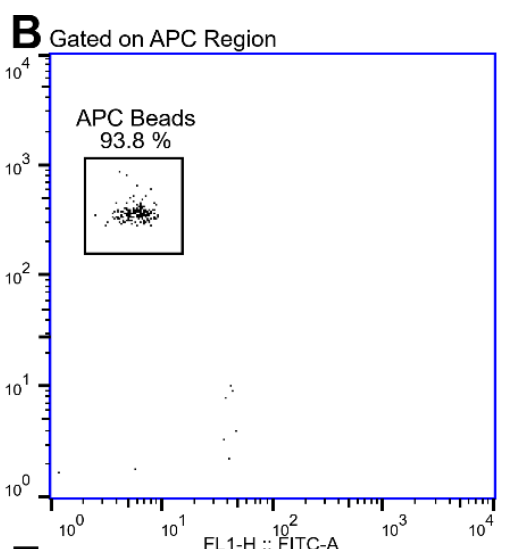
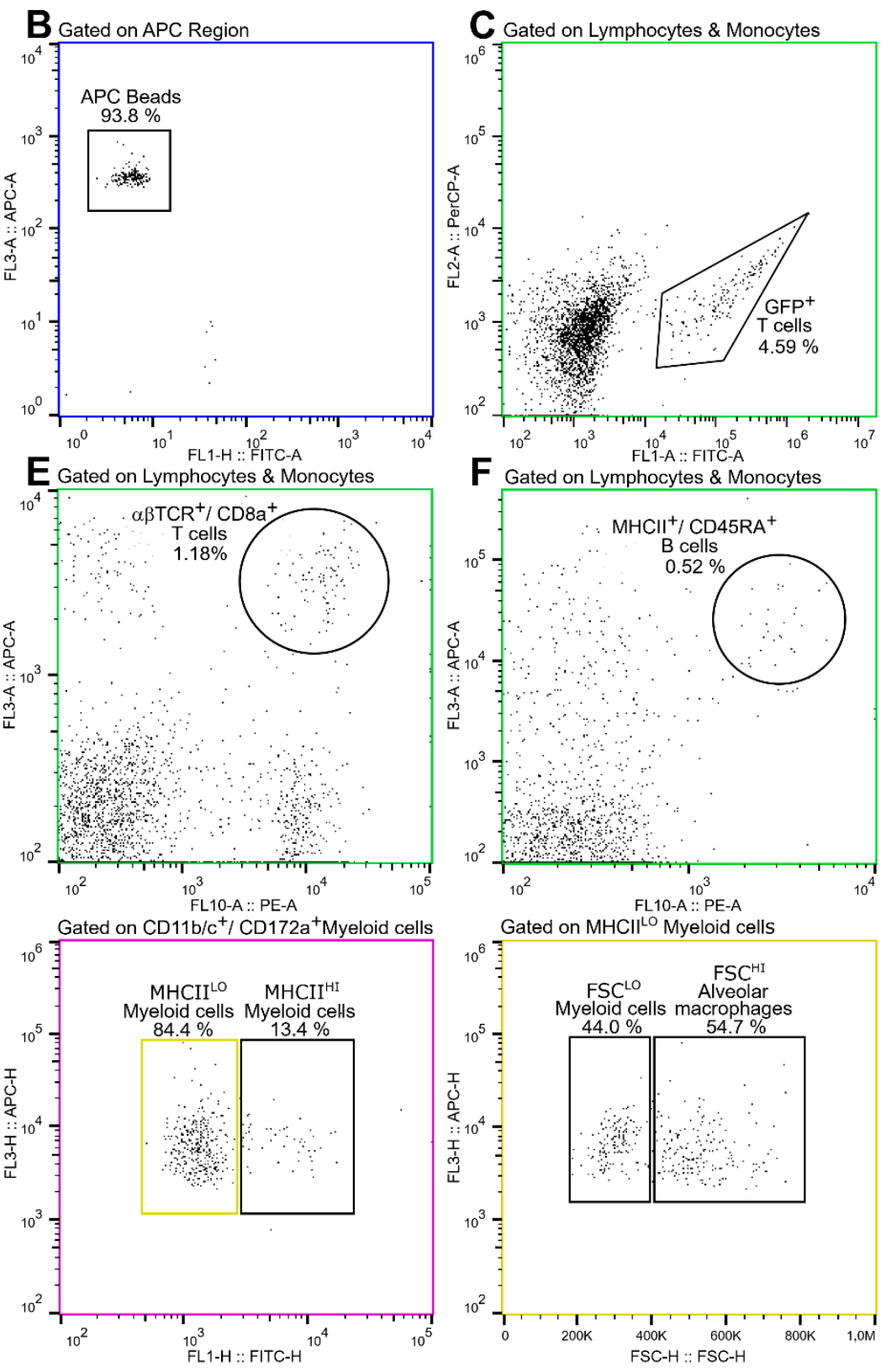

Figure 5: Gating strategy for flow cytometry acquisition of different immune cell populations. Depicted are the exemplified gating strategies used for the analysis and quantification of different immune cell types. (A) General gating strategy to roughly distinguish lymphocytes and monocytes from granulocytes and APC beads. (B) Gating for APC beads used for the quantification of cell populations. (C) Gating for GFP+ $T_{M B P}$ cells. (D - H) Gating strategies and surface marker combinations used for antibody labeling of (D) CD4 ${ }^{+} T$ cells, (E) CD8a+ $T$ cells, (F) B cells, (G) different subgroups of myeloid cells and (H) neutrophils. The gating strategy is color-coded: identical colors of gate and frame indicate parent population and subpopulation, respectively. The antibodies used for cell surface marker stainings are listed in Table 1. 

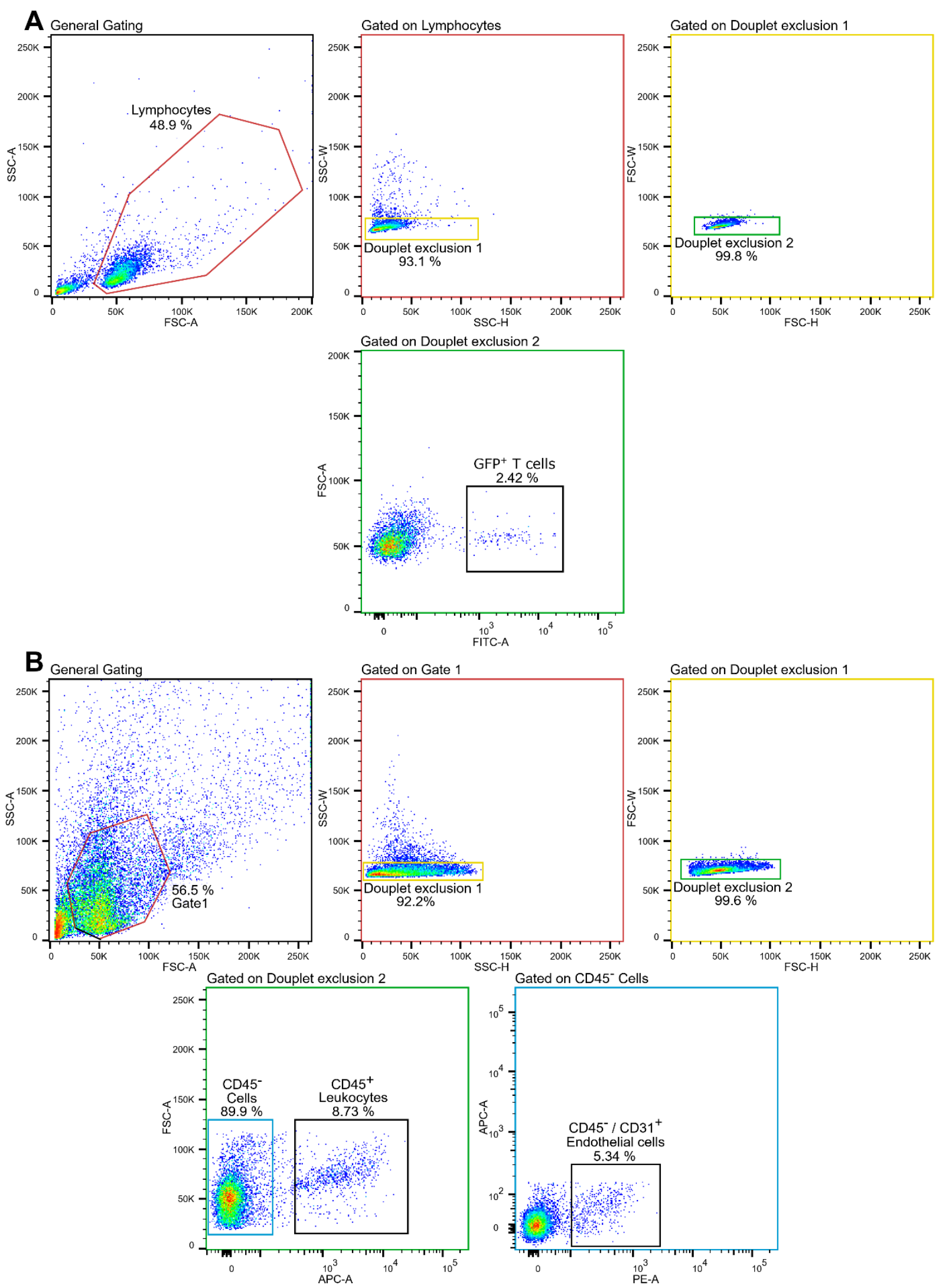

Figure 6: Gating strategy for fluorescence-activated sorting of GFP+ $T_{M B P}$ cells and spinal cord endothelial cells. Depicted are the exemplified gating strategies used for the flow cytometry sorting of (A) GFP+ $T_{M B P}$ cells and (B) spinal cord endothelial cells. The gating strategy is color-coded: identical colors of gate and frame indicate parent population and subpopulation, respectively. The antibodies used for cell surface marker stainings are listed in Table 1. 


\subsubsection{RNA isolation, cDNA synthesis and quantitative real-time PCR}

Gene expression on transcription level was determined using quantitative real-time PCR (qPCR). RNA isolation from sorted T cells or total tissues that had been stored in TRI Reagent (Merck, Germany) at $-80{ }^{\circ} \mathrm{C}$ was performed according to the manufacturer's instructions. Reverse transcription into cDNA was performed using the RevertAid First Strand CDNA synthesis kit (Thermo Fisher Scientific, USA) according to the manufacturer's instructions. qPCR was performed on an Applied Bioystems StepOnePlus Real-Time PCR system (Thermo Fisher Scientific, USA) using target-specific TaqMan probes quenched with TAMRA and labeled with FAM (Thermo Fisher Scientific, USA). $\beta$-actin served as house-keeping gene. All measurements were performed in duplicates. The following primers and probes were used:

Table 5: List and sequences of qPCR primers

\begin{tabular}{|c|c|c|c|}
\hline Gene (Abbreviation) & Forward primer $\left(5^{\prime}-3^{\prime}\right)$ & Reverse primer $\left(5^{\prime}-3^{\prime}\right)$ & Probe (FAM-5'-3'-TAMRA) \\
\hline$\beta$-actin $(A c t b)$ & $\begin{array}{l}\text { GTACAACCTCCTTGCAG } \\
\text { СТCCT }\end{array}$ & $\begin{array}{l}\text { TTGTCGACGACGAG } \\
\text { CGC }\end{array}$ & $\begin{array}{l}\text { CGCCACCAGTTCGCCATG } \\
\text { GAT }\end{array}$ \\
\hline Interferon gamma (Ifng) & $\begin{array}{l}\text { AACAGTAAAGCAAAAA } \\
\text { AGGATGCATT } \\
\end{array}$ & $\begin{array}{l}\text { TTCATTGACAGCTTT } \\
\text { GTGCTGG }\end{array}$ & $\begin{array}{l}\text { CGCCAAGTTCGAGGTGA } \\
\text { ACAACCC }\end{array}$ \\
\hline Interleukin-6 (//6) & $\begin{array}{l}\text { GTTGCCTTCTTGGGA } \\
\text { CTGAT }\end{array}$ & $\begin{array}{l}\text { TGAAGTCTCCTCTCC } \\
\text { GGACT }\end{array}$ & $\begin{array}{l}\text { TGTTGACAGCCACTG } \\
\text { CCTTCCC }\end{array}$ \\
\hline Interleukin-12 (//12) & $\begin{array}{l}\text { GAACTACAGCACCAG } \\
\text { CTTCTTCATC }\end{array}$ & $\begin{array}{l}\text { CTTCAAAGGTTTCAC } \\
\text { CTGCAGG }\end{array}$ & $\begin{array}{l}\text { ACATCATCAAACCGGACC } \\
\text { CACCCAA }\end{array}$ \\
\hline Interleukin-17A (//17a) & $\begin{array}{l}\text { GAGTCCCCGGAGAATTC } \\
\text { CAT }\end{array}$ & $\begin{array}{l}\text { GAGTACCGCTGCCTT } \\
\text { CACTGT }\end{array}$ & ATGTGCCTGATGCTGTT \\
\hline $\begin{array}{l}\text { Tumor necrosis factor } \alpha \\
(T N F a)\end{array}$ & $\begin{array}{l}\text { TCGAGTGACAAGCCC } \\
\text { GTAGC }\end{array}$ & $\begin{array}{l}\text { CTCAGCCACTCCAGC } \\
\text { TCCTC }\end{array}$ & $\begin{array}{l}\text { CGTCGTAGCAAACCACCA } \\
\text { AGCAGA }\end{array}$ \\
\hline $\begin{array}{l}\text { Major histocompatibility } \\
\text { complex II (Mhcll) }\end{array}$ & $\begin{array}{l}\text { GGTTGAGAACAGCAAGC } \\
\text { CAGTC }\end{array}$ & $\begin{array}{l}\text { GGTGAGGTAAGCCATC } \\
\text { TTGTGG }\end{array}$ & $\begin{array}{l}\text { TGAGACCAGCTTCCTTTCCA } \\
\text { ACCCTGA }\end{array}$ \\
\hline $\begin{array}{l}\text { C-X-C chemokine receptor } \\
\text { type } 3\left(C_{x c r} 3\right)\end{array}$ & $\begin{array}{l}\text { AGCAGCCAAGCCATGT } \\
\text { ACCTT }\end{array}$ & $\begin{array}{l}\text { TAGGGAGATGTGCT } \\
\text { GTTTTCCA }\end{array}$ & $\begin{array}{l}\text { AGGTCAGTGAACGTCAA } \\
\text { GTGC TAGATGCCTC }\end{array}$ \\
\hline $\begin{array}{l}\text { C-X-C chemokine receptor } \\
\text { type } 4(C x c r 4)\end{array}$ & $\begin{array}{l}\text { GGAGGTCATCAAGCAAG } \\
\text { GATGT }\end{array}$ & $\begin{array}{l}\text { GGGTTCAGGCAACA } \\
\text { GTGGA }\end{array}$ & $\begin{array}{l}\text { TTCGAGAGCGTCGTGCAC } \\
\text { AA }\end{array}$ \\
\hline $\begin{array}{l}\text { C-C chemokine receptor } \\
\text { type } 5(C c r 5)\end{array}$ & $\begin{array}{l}\text { GTTCTCCTGTGGACCGG } \\
\text { GTATACG }\end{array}$ & $\begin{array}{l}\text { ATTGTCAAACGCTTC } \\
\text { TGCAAAC }\end{array}$ & $\begin{array}{l}\text { AGCTTACACGATCAGGA } \\
\text { TT }\end{array}$ \\
\hline $\begin{array}{l}\text { C-C chemokine receptor } \\
\text { type } 6(\mathrm{Ccr} 6)\end{array}$ & $\begin{array}{l}\text { GGCCGGAACATTATT } \\
\text { GGAAA }\end{array}$ & $\begin{array}{l}\text { TTCCAGAGACCGAGC } \\
\text { CATG }\end{array}$ & $\begin{array}{l}\text { TGAATTTCACCGAGGCCA } \\
\text { ACTACGGA }\end{array}$ \\
\hline $\begin{array}{l}\text { Integrin } \alpha 4 \beta 1 \text { / Very late } \\
\text { antigen-4 (Vla4) }\end{array}$ & $\begin{array}{l}\text { GAAGGAAGAGTGTTCG } \\
\text { TGTACATCA }\end{array}$ & $\begin{array}{l}\text { CGACGAGCACTCTTT } \\
\text { CCATTT }\end{array}$ & $\begin{array}{l}\text { CTCTGGCATGGGAGCTGT } \\
\text { GATGGT }\end{array}$ \\
\hline $\begin{array}{l}\text { Lymphocyte function- } \\
\text { associated antigen-1 } \\
\text { (Lfa1) }\end{array}$ & $\begin{array}{l}\text { CTGCTACTCATCCTA } \\
\text { GCTGGACTACTC }\end{array}$ & $\begin{array}{l}\text { GCAGTTGCTGACTTT } \\
\text { GTACTTGGT }\end{array}$ & $\begin{array}{l}\text { TCCTGGGATCTGCCCTGT } \\
\text { CC }\end{array}$ \\
\hline $\begin{array}{l}\text { Vascular cell adhesion } \\
\text { protein-1 (Vcam1) }\end{array}$ & $\begin{array}{l}\text { ACATGAGGGTGCTCC } \\
\text { TGTGA }\end{array}$ & $\begin{array}{l}\text { GGTGGCATTTCCCGA } \\
\text { GAGGA }\end{array}$ & $\begin{array}{l}\text { TGTGCCAGCGAGGGT } \\
\text { CTACCAGCTCCT }\end{array}$ \\
\hline $\begin{array}{l}\text { Intercellular adhesion } \\
\text { molecule-1 (Icam) }\end{array}$ & $\begin{array}{l}\text { GGAGACAGCAGACCA } \\
\text { CTGTGCTT }\end{array}$ & $\begin{array}{l}\text { CTCGCTCTGGGAACG } \\
\text { AATACA }\end{array}$ & ACTGTGGCACCACGC \\
\hline Claudin-5 (Cldn5) & $\begin{array}{l}\text { CGGGCGTCCAGAGTTC } \\
\text { AGT }\end{array}$ & $\begin{array}{l}\text { TAGACGTAGTTCTTC } \\
\text { TTGTCGTAATCG }\end{array}$ & $\begin{array}{l}\text { CCAGTCAAGTACTCA } \\
\text { GCACCAAGGCGA }\end{array}$ \\
\hline Occludin (Ocln) & $\begin{array}{l}\text { CCTAATGTGGAAGAG } \\
\text { TGGGTTAAAAA }\end{array}$ & $\begin{array}{l}\text { GTCGACTCTTTCCGC } \\
\text { ATAGTCA }\end{array}$ & $\begin{array}{l}\text { CACACAAGACATGCCTCC } \\
\text { ACCCCC }\end{array}$ \\
\hline
\end{tabular}




\subsubsection{Next-Generation Sequencing of sorted T cells}

Next-generation sequencing (NGS) of the transcriptome of sorted $\mathrm{T}_{\mathrm{MBP}}$ cells was performed at the Transcriptome and Genome Analysis Laboratory (TAL) of the University Medical Center Göttingen (UMG) under supervision of Dr. Gabriela Salinas. Sequencing data analysis was performed by Dr. Orr Shomroni. Additional statistical analysis and graphical depiction was performed by Dr. Francesca Odoardi, Michael Haberl and Leon Hosang using Microsoft Excel (2010, 2016; Microsoft, USA) and GraphPad Prism (V8; GraphPad, USA). Principle component analysis (PCA) was performed using R (V3.6.0; R Core Team). Biological processes Gene Ontology (GO) term enrichment analysis was performed using DAVID (V6.8; LHRI, USA).

\subsubsection{Statistical analysis}

Statistical analysis was performed using GraphPad Prism (V6 - 8; GraphPad, USA) and Microsoft Excel (2010 and 2016; Microsoft, USA). Unless differently indicated, data are represented as mean \pm SEM (standard error of the mean). The statistical tests underlying data analysis are stated in the corresponding figure legends. In some graphs, the data from two independent experiments were combined provided they were not significantly different. To this end, Gaussian distribution of each independent set of data was tested using a Kolmogorov-Smirnov test with Lilliefors' correction. In case of normal distribution, intergroup comparisons were analyzed using two-tailed unpaired $t$-tests. In case the number of animals per experiment was not sufficient to test for Gaussian distribution or the data were not normally distributed, intergroup comparisons were performed using the non-parametric Mann-Whitney test. Data of two experiments were only combined if no significant difference was observed.

Significance levels were set as ${ }^{*} p<0.05 ;{ }^{* *} p<0.01 ;{ }^{* * *} p<0.001$. 


\section{Results}

\section{1. $T_{M B P-E M}$ cell activation and EAE induction via the lung}

\subsubsection{Establishment and characterization of an i.tr. induced EAE model}

\subsubsection{Intratracheal immunization with low doses of MBP induces EAE}

In order to examine the potential role of the lung in triggering CNS autoimmunity, we first aimed to establish an aEAE model based on the activation of MBP-specific T effector memory ( $T_{M B P-E M}$ ) cells within the lung. To this end, $7.5 \times 10^{6} \mathrm{~T}_{\text {MBP-EM }}$ cells (resting $T_{M B P}$ cells, D6 after in vitro antigen encounter) were i.v. transferred into naïve recipient animals. $6-12 \mathrm{~h}$ later, the rats were i.tr. immunized with MBP emulsified in CFA (Fig. 7A).

The CFA dose commonly used for s.c. immunization in aEAE is $150 \mu \mathrm{g} /$ animal (average body weight: $150-200 \mathrm{~g}$ ). For i.tr. immunization, a starting dose of $50 \mu \mathrm{g} / 300 \mathrm{~g}$ body weight was chosen because it was well-tolerated by the animals. The standard MBP concentration used for s.c. immunization in aEAE is $75 \mu \mathrm{g} /$ animal. Limited by instillation volume and MBP stock concentration, we chose a maximum dose of $50 \mu \mathrm{g} \mathrm{MBP} / 300 \mathrm{~g}$ body weight as starting point. We then performed a dose titration in order to identify the most effective antigen dose. Therefore, the animals were i.tr. immunized with MBP doses ranging from $0.01 \mu \mathrm{g}$ to $50 \mu \mathrm{g} /$ $300 \mathrm{~g}$ body weight emulsified in $50 \mu \mathrm{g}$ CFA / $300 \mathrm{~g}$ body weight $6-12 \mathrm{~h}$ following $\mathrm{T}_{\text {MBP-EM cell }}$ transfer.

On D1 post-immunization (p.i.), all tested groups showed body weight losses between $6-10$ \%. Between D2 and D3 p.i., the weight of all groups started to increase again (Fig. 7B). While the body weight of the groups immunized with the lowest ( 0 and $0.01 \mu \mathrm{g} / 300 \mathrm{~g}$ body weight) and the highest MBP concentrations ( $50 \mu \mathrm{g} / 300 \mathrm{~g}$ body weight) steadily increased over the following days, the body weight of the other groups (0.1, 1 and $10 \mu \mathrm{g} \mathrm{MBP} \mathrm{/} 300 \mathrm{~g}$ body weight) began to stagnate and slightly decrease on D4 p.i. and during the following $2-3$ days (Fig. 7B). Coinciding with this second period of weight loss, some animals began to exhibit first clinical symptoms between D5 and D6 p.i. (Fig. 7D).

Interestingly, only the animals immunized with MBP at a dose of $1 \mu \mathrm{g} / 300 \mathrm{~g}$ body weight developed a severe EAE with an incidence of $100 \%$ (Fig. 7C) and an average peak score of 2.7 \pm 0.15 on D7 p.i. (Fig. 7E).

Mild EAE with peak scores of slightly less than 1 and reduced incidence was induced in the groups immunized with $10 x$ higher $(75 \%$ incidence, peak score $0.88 \pm 0.38$ ) and $10 x$ lower (50 $\%$ incidence, peak score $0.88 \pm 0.41$ ) MBP concentrations (Fig. 7C, E).

The highest MBP concentration tested ( $50 \mu \mathrm{g} / 300 \mathrm{~g}$ body weight) resulted in the induction of EAE in merely $40 \%$ of animals (Fig. 7C). In these animals, EAE was significantly delayed and its severity was particularly low: Clinical symptoms started between D7 and D8 p.i. (Fig. 7D) and the average peak score was $0.55 \pm 0.54$ (Fig. 7E). In all groups the clinical symptoms vanished by D11 p.i. 
A

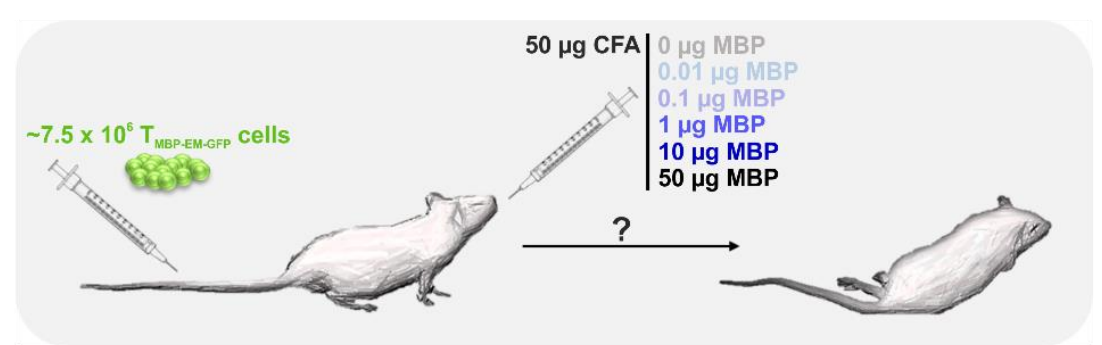

B
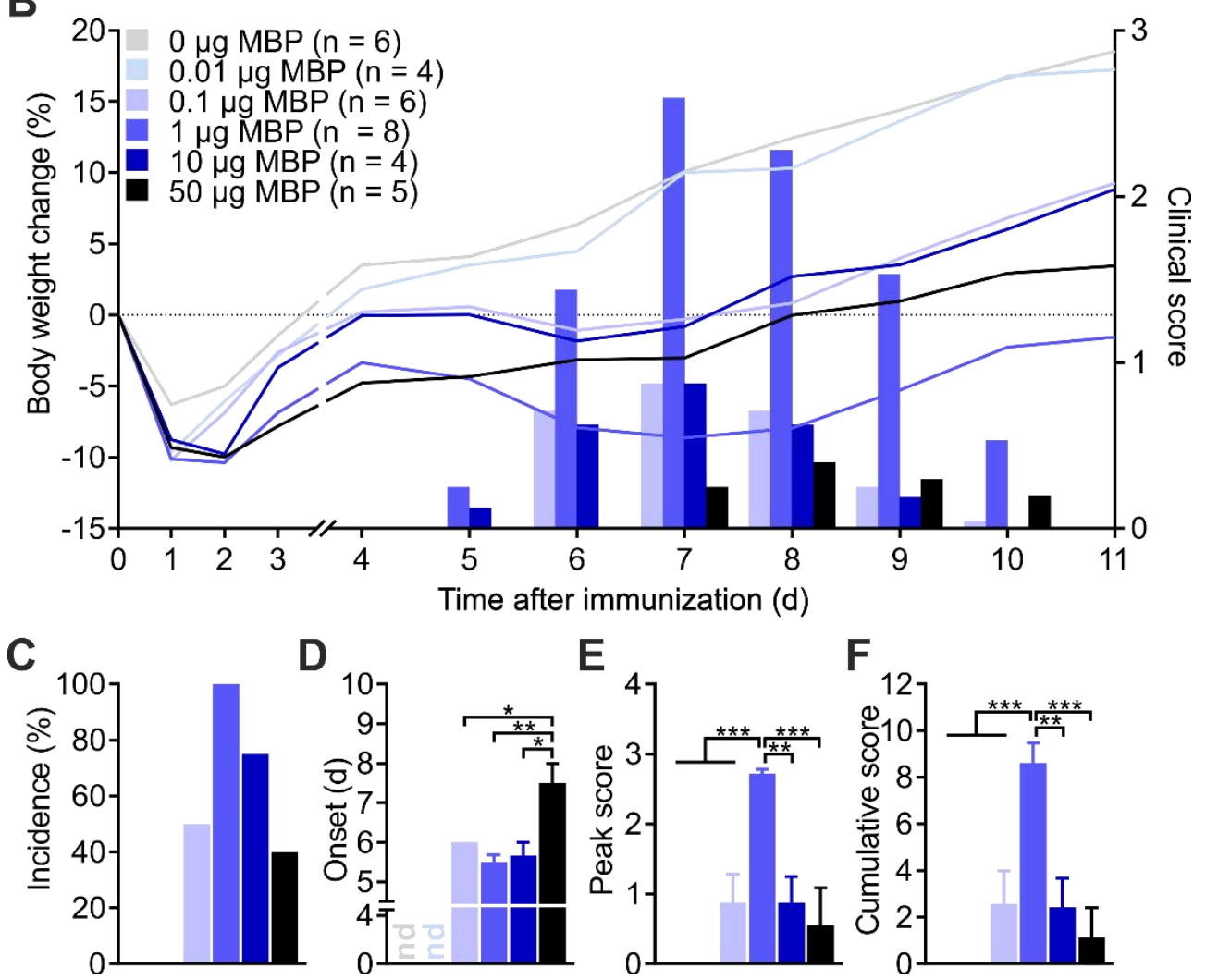

F

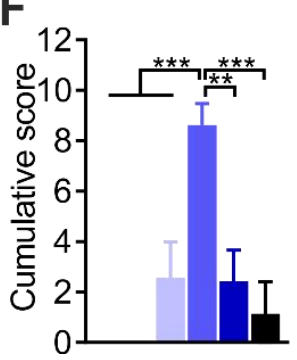

Figure 7: Titration of the MBP concentration used for i.tr. immunization. (A) Experimental design: Rats received $7.5 \times 10^{6}$

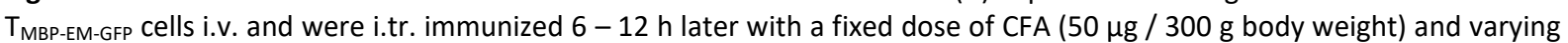
doses of $\operatorname{MBP}(0,0.01,0.1,1,10,50 \mu \mathrm{g} / 300 \mathrm{~g}$ body weight). (B) Body weight change (lines) and clinical scores (bars) over the course of EAE. (C) Incidence (\%). (D) Average onset (days p.i.). (E) Average peak score. (F) Average cumulative score. Cumulative data from five independent experiments. Values are represented as mean \pm SEM. Statistical significance was determined via one-way ANOVA with Tukey's multiple comparisons test. ${ }^{*} p<0.05 ;{ }^{* *} p<0.01 ;{ }^{* * *} p<0.001$.

\subsubsection{Moderate CFA concentrations are sufficient to induce EAE via the lung}

In order to optimize the protocol, we next titrated the CFA concentration used for i.tr. immunization. To this end, the rats were i.tr. immunized with the previously determined optimal MBP dose ( $1 \mu \mathrm{g} / 300 \mathrm{~g}$ body weight) and varying CFA concentrations ranging from 50 $\mu \mathrm{g}$ down to $1 \mu \mathrm{g} / 300 \mathrm{~g}$ body weight following TMBP-EM cell transfer.

Between D4 and D5 p.i., the 50, 25, 10 and $5 \mu \mathrm{g}$ CFA groups started exhibiting weight stagnation or loss, preceding the onset of first clinical symptoms starting between D5 and D6 p.i. (Fig. $8 \mathrm{~A}-\mathrm{C}$ ). In the subsequent days, a severe EAE developed in all 4 groups, reaching average peak scores between 2.58 and 2.81 on D7 p.i. and average cumulative scores ranging from 9.5 to 11.4 (Fig. 8D - E). On D8 / D9 p.i., clinical symptoms started to decrease and the 
animals fully recovered by D11 p.i. (Fig. 8A). Animals treated with 1 and $0 \mu \mathrm{g}$ CFA did not show any signs of EAE.

In summary, we observed that very low doses of antigen ( $75-150$-fold reduced compared to the dose used for s.c. immunization) and of adjuvant ( $15-30$-fold reduced compared to the dose used for s.c. immunization) can induce a severe EAE when delivered i.tr. Mild EAE could still be induced with 750 - 1500-fold reduced doses of MBP compared to s.c. immunization. These findings suggest that the lung is not just capable but also very efficient in triggering CNS autoimmunity.
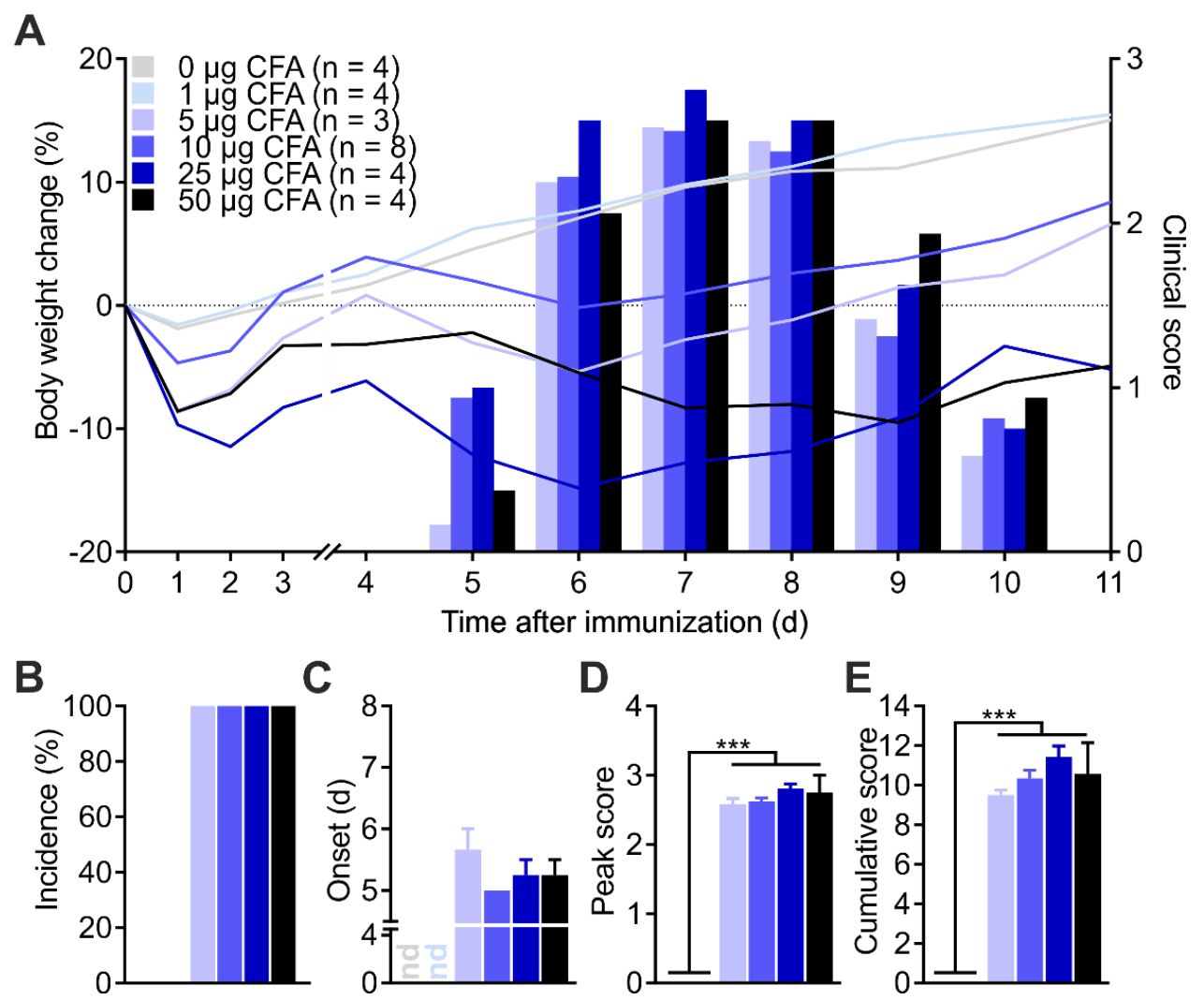

Figure 8: Titration of the CFA concentration used for i.tr. immunization. Rats received $7.5 \times 10^{6} \mathrm{~T}_{\mathrm{MBP} \text {-EM-GFP }}$ cells i.v. and were i.tr. immunized $6-12 \mathrm{~h}$ later with a fixed dose of MBP ( $1 \mu \mathrm{g} / 300 \mathrm{~g}$ body weight) and varying doses of CFA $(0,1,5,10,25,50$ $\mu \mathrm{g} / 300 \mathrm{~g}$ body weight). (A) Body weight change (lines) and clinical scores (bars) over the course of EAE. (B) Incidence (\%). (C) Average onset (days p.i.). (D) Average peak score. (E) Average cumulative score. Cumulative data from four independent experiments. $\mathrm{nd}=$ not determined. Values are represented as mean $\pm \mathrm{SEM}$. Statistical significance was determined via oneway ANOVA with Tukey's multiple comparisons test. ${ }^{* * *} p<0.001$.

\subsubsection{Immunization with a low MBP concentration induces EAE in memory rats}

The newly established protocol of $\mathrm{T}_{\text {MBP-EM }}$ cell activation via the lung (lung EAE model) is based on a relatively high number of transferred T cells $\left(7.5 \times 10^{6}\right)$ and a relatively short time interval between transfer and immunization $(6-12 \mathrm{~h})$. To test whether the highly reduced doses of antigen and adjuvant ( $1 \mu \mathrm{g}$ MBP and $10 \mu \mathrm{g}$ CFA / $300 \mathrm{~g}$ body weight) could trigger CNS disease in a more physiological setup, we i.tr. immunized a group of memory rats (Fig. 9A) in which

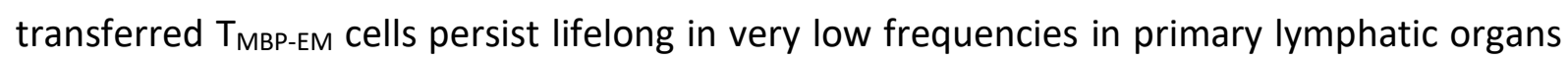
but also in the lung (Kawakami et al., 2005). 
In line with the previous results, all animals developed EAE. After an initial weight loss of $8 \%$ following immunization, the animals' body weight started to increase again between D2 and D4 p.i., before a second episode of weight loss lasting until D9 p.i. started. This second episode of weight loss was accompanied by the emergence of first clinical symptoms on D6 p.i. EAE reached its average peak score of $2.25 \pm 0.14$ on $D 7$ p.i. before symptoms started vanishing again. The animals fully recovered by D12 p.i (Fig. 9B).

A

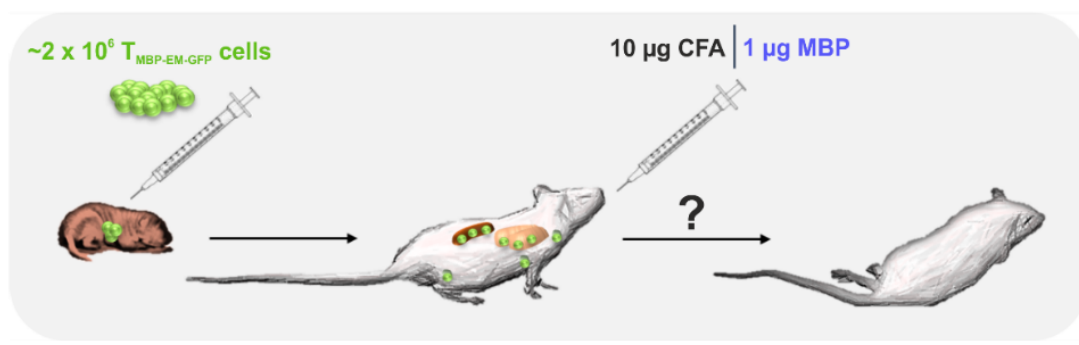

B

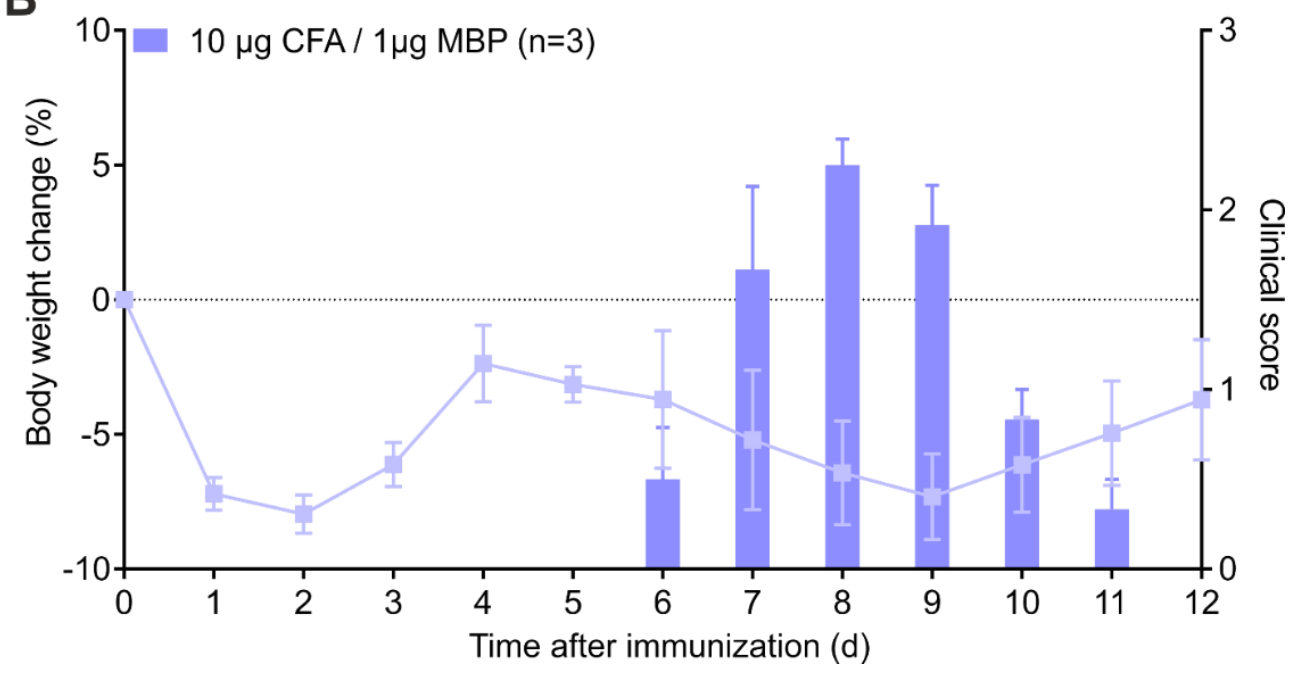

Figure 9: I.tr. immunization of memory rats with a low MBP dose. (A) Experimental design: Memory rats were generated by

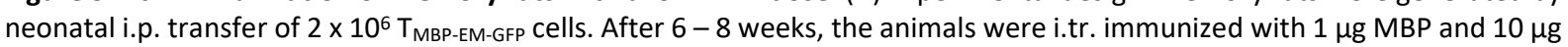
CFA / 300 g body weight. (B) Body weight change (lines) and clinical scores (bars) over the course of EAE.

\subsubsection{Kinetic of $T_{M B P}$ cell distribution during i.tr. induced $E A E$}

We went on to further characterize the newly established lung EAE model. To this end, distribution (Figs. 10 and 11) and activation state (Figs. 10 and 12) of transferred T cells in different organs was determined on different days following i.tr. immunization. Based on the previous clinical results (Fig. 7), animals were immunized with the following antigen doses: 50

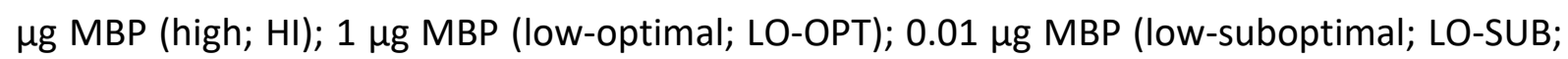
all concentrations per $300 \mathrm{~g}$ body weight). Furthermore, we included two control groups: one group received T cells without being immunized (naïve; NAl); the other group was immunized with CFA only (CFA). Animals were sacrificed before immunization (D0; $12 \mathrm{~h}$ p.t.), on D1 (expected time point of $\mathrm{T}$ cell activation), D3 (expected time point of ongoing $\mathrm{T}$ cell proliferation and migration), D5 (onset of disease), and D7 (peak of disease) p.i. 


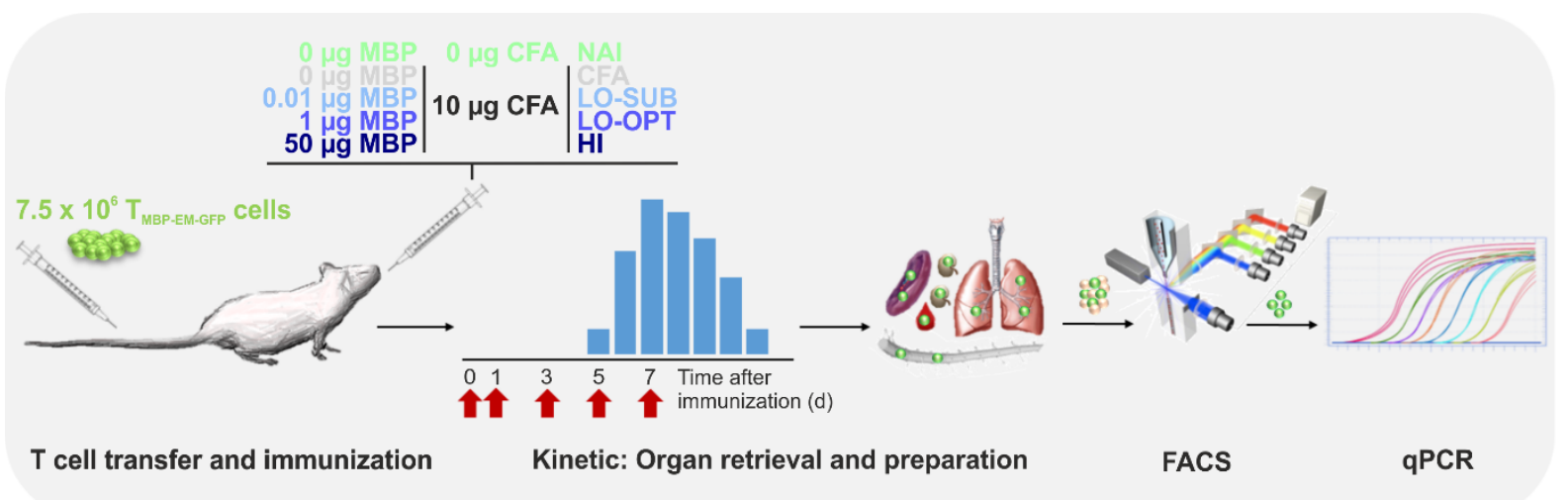

Figure 10: Experimental scheme for the kinetic of $T_{M B P}$ cell distribution during i.tr. induced EAE. The animals received $7.5 \mathrm{x}$ $10^{6} \mathrm{~T}_{\text {MBP-EM-GFP }}$ cells and were i.tr. immunized 6-12 $\mathrm{h}$ later with the indicated combinations of MBP and CFA (per $300 \mathrm{~g}$ body weight). Lung, mediastinal and inguinal lymph nodes, spleen blood and spinal cord (CNS) were removed on D0, D1, D3, D5 and D7 p.i. The organs were processed and the amount of transferred T cells determined using flow cytometry. T cells were additionally sorted from lung, mediastinal lymph nodes, blood and spinal cord. The expression of IFN $\gamma$ and IL-17 was determined as read-out for activation using qPCR. NAI = naïve; CFA = adjuvant only; LO-SUB = low-suboptimal MBP dose; LO$\mathrm{OPT}=$ low-optimal MBP dose; $\mathrm{HI}=$ high MBP dose.

As expected based on the previous results, a severe EAE was only induced in the animals immunized under LO-OPT antigen conditions. The LO-SUB and HI group only developed mild EAE characterized by a reduced incidence, delayed onset and a low average peak score (Fig. $11 A-D)$.

FACS quantification of transferred T cells in the different organs revealed that $T$ cell numbers in the peripheral organs generally increased in a dose-dependent manner with the highest number of cells observed in the HI group. In striking contrast, in the spinal cord, the highest number of infiltrated T cells was observed in the LO-OPT group (Fig. 11E - K).

In more detail, the number of transferred T cells in the lung on D1 p.i. was low and comparable between the different groups. First significant differences started to emerge on D3 p.i. While $\mathrm{T}$ cell numbers in all groups gradually increased, the highest numbers were observed in the $\mathrm{HI}$ group, followed by the LO-OPT group. T cell numbers reached their peak on D5 p.i., and the antigen dose-dependent increase became even more obvious. On D7 p.i., the trend of a dosedependent $T$ cell number continued, though cell numbers in general were going down again (Fig. 11E).

In the lung-draining mediastinal lymph nodes, barely any cells could be observed in any of the groups on D0 and D1 p.i. On D3 p.i., a strong and dose-dependent increase could be observed in the LO-OPT and the HI group. In comparison, only few T cells were found in the LO-SUB, CFA and NAI groups. On D5 and D7 p.i., T cell numbers in the mediastinal lymph nodes of the $\mathrm{HI}$ and the LO-OPT group decreased again but remained higher than in the other groups (Fig. $11 \mathrm{~F})$.

In the non-draining inguinal lymph nodes, a gradual increase in T cell numbers was observed over the course of the experiment. This increase was comparable between the experimental groups, with a slight tendency toward a higher number in the HI group (Fig. 11G).

In the spleen, very few T cells were detectable in all groups on D0 and D1 p.i. On D3 p.i., T cell numbers started to increase in a dose-dependent manner and peaked on D5 p.i., shortly before EAE onset. The by far highest number of T cells was observed in the spleen of the $\mathrm{HI}$ 
group, followed by the LO-OPT group. The T cell number of the LO-SUB group was by trend but not significantly higher than in the CFA and NAI group. On D7 p.i., the cell numbers had again decreased to a comparable number in all groups but the $\mathrm{HI}$ group (Fig. $11 \mathrm{H}$ ).

In the blood only few cells could be observed in all groups on D0 and D1 p.i. Starting on D3 p.i., T cell numbers started increasing in an MBP dose-dependent manner. Cell numbers in all groups peaked on D5 p.i., just before the onset of EAE. The highest $T$ cell numbers were observed in the HI group followed by the LO-OPT group whereas numbers in the NAI, CFA and LO-SUB groups were comparable. On D7 p.i., the T cell numbers of all groups had decreased to a similarly low level with exception of the LO-SUB group, which showed slightly increased numbers (Fig. 11I).

Between D0 and D3 p.i., no T cells were observed in the spinal cord of any of the groups. On D5 and D7 p.i., T cells were detected in spinal cords of the LO-SUB, LO-OPT and HI but not the NAI and CFA groups. Despite the high number of cells observed in the blood of the HI group on D5 p.i., only a minor proportion entered the spinal cord (Fig. 11J, K). In contrast and in line with the clinical course of EAE, the T cell number was almost 10x higher in the LO-OPT group. On D7 p.i., the T cell numbers detected in the CNS of the LO-SUB and the LO-OPT group remained similar compared to $D 5$ p.i. In contrast, $T$ cell infiltration into the CNS of the HI group further increased (Fig. 11J, K). No T cells could be detected in the CNS of the NAI and the CFA group at any of the examined time points (Fig. 11J, K). 
A

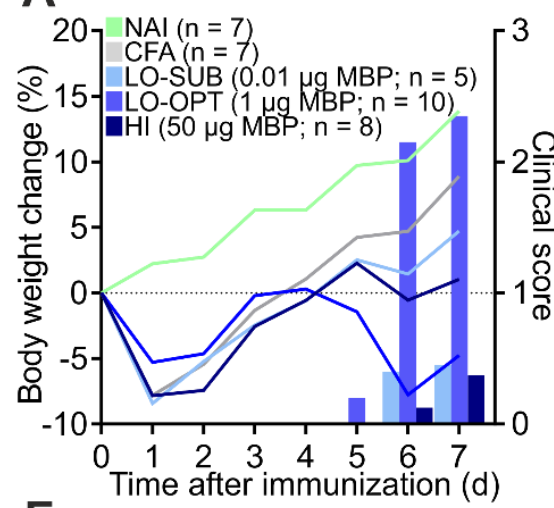

E

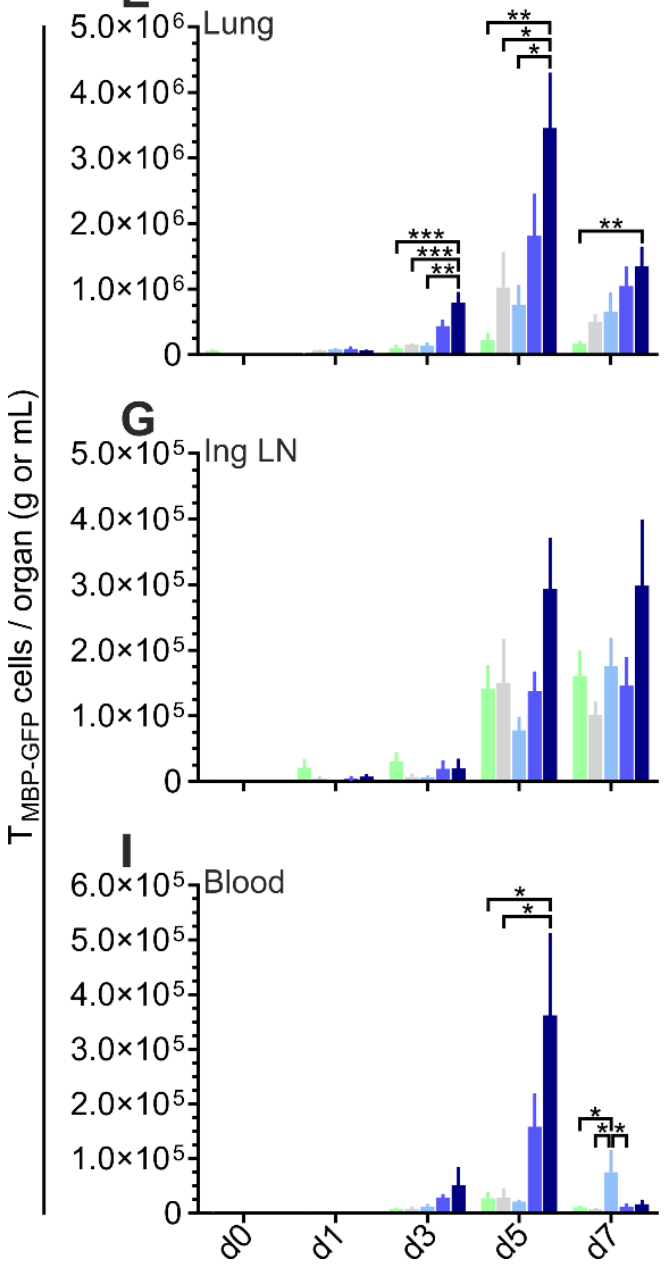

B

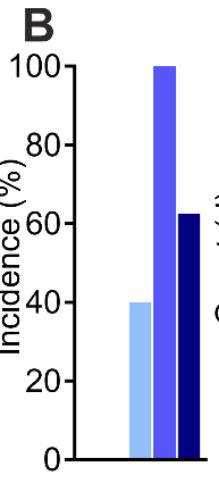

C

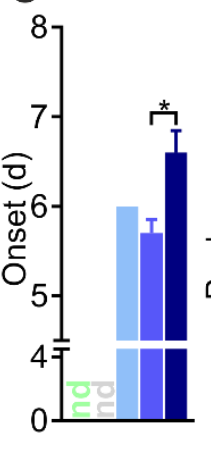

D

$\mathbf{F}$

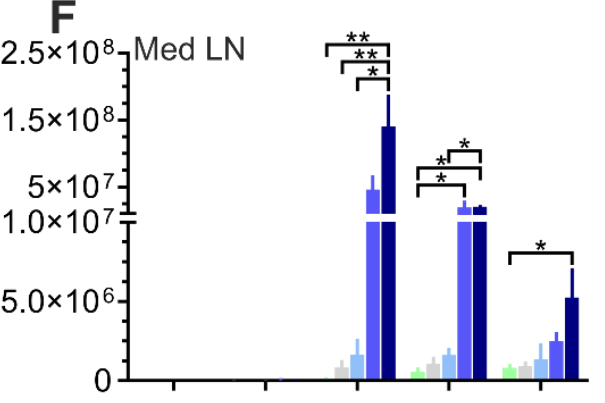

H
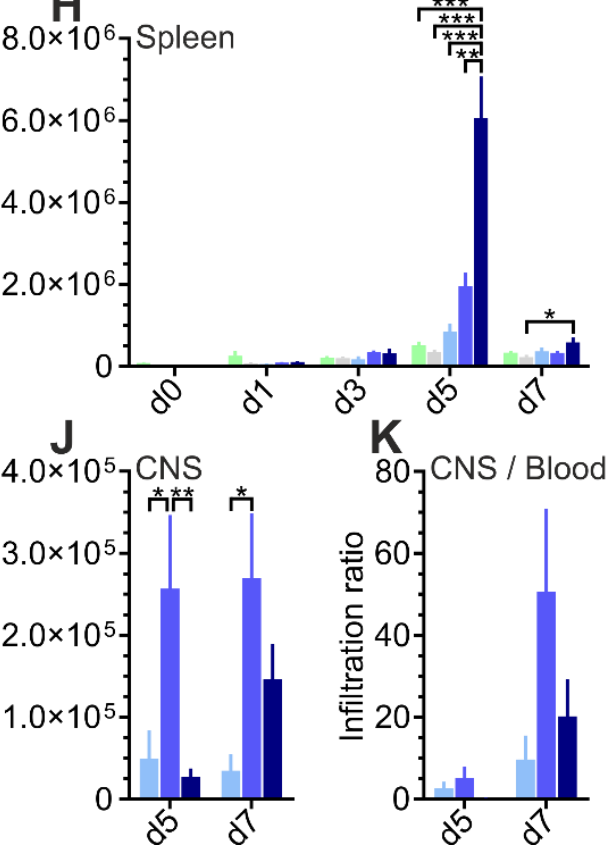

Figure 11: Characterization of $\mathrm{T}_{\mathrm{MBP}}$ cell distribution in different organs during i.tr. induced EAE. Rats received $7.5 \times 10^{6} \mathrm{~T}_{\mathrm{MBP}}$ EM-GFP cells and were i.tr. immunized $6-12 \mathrm{~h}$ later with varying doses of MBP (0 $\mu \mathrm{g}$ : NAI and CFA; $0.01 \mu \mathrm{g}$ : LO-SUB; $1 \mu \mathrm{g}$ : LOOPT; $50 \mu \mathrm{g}$ : $\mathrm{HI}$; all per $300 \mathrm{~g}$ body weight) and CFA ( $0 \mu \mathrm{g}$ : NAl; $10 \mu \mathrm{g}$ : rest; all per $300 \mathrm{~g}$ body weight). (A) Body weight change (lines) and clinical scores (bars) over the course of EAE. (B) Incidence (\%). (C) Average onset (days p.i.). (D) Average peak score. $(E-J)$ Transferred $T$ cells per $g$ or $\mathrm{mL}$ detected in the indicated organs at indicated time points $p . i$. (K) Infiltration rate: $T$ cells in spinal cord (CNS; /g) / T cells in blood (/mL) on D5 and 7 p.i. Cumulative data from three independent experiments. Number of animals for the EAE clinical outcome as indicated. Number of animals for T cell quantification: $4-8$ / group and time point. $\mathrm{nd}=$ not determined. Values are represented as mean $\pm \mathrm{SEM}$. Statistical significance was determined via one-way ANOVA with Tukey's multiple comparisons test. ${ }^{*} \mathrm{p}<0.05 ;{ }^{* *} \mathrm{p}<0.01 ;{ }^{* * *} \mathrm{p}<0.001$. 


\subsubsection{Kinetic of $T_{M B P}$ cell activation during i.tr. induced $E A E$}

In addition to their distribution, we determined the activation state of the transferred $T$ cells at different time points in different organs throughout the course of i.tr. induced EAE. The experimental setup was identical to the previous part (see 3.1.1.4 and Fig. 10).

On D1 p.i., a clear upregulation of both IFN $\gamma$ and IL-17 expression was observed in T cells from the lung of the $\mathrm{HI}$ group. The expression of both genes was significantly higher than in the NAI, CFA and LO-SUB but not the LO-OPT group (Fig. 12A). IFN $\gamma$ and IL-17 expression of T cells sorted from lungs of the LO-OPT group also tended to be higher than in the NAI, CFA and LOSUB group. This difference, however, was not significant (Fig. 12A). On D3 p.i., expression of both IFN $\gamma$ and IL-17 in the $\mathrm{HI}$ and the LO-OPT group had decreased again and was indistinguishable from the NAI, CFA and LO-SUB groups (Fig. 12A).

Barely any T cells could be observed in or sorted from the mediastinal lymph nodes on D0 and D1 p.i. Expression of both IFN $\gamma$ and IL-17 on D3 and D5 p.i. was decreased by a factor of around 10 compared to T cells sorted from the lung at the same time points (Fig. 12B). A significantly higher expression of IFN $\gamma$ was detected in T cell from the NAI group compared to the other groups on D3 p.i. (Fig. 12B). Apart from this, the expression of IFN $\gamma$ and IL-17 was similar between the different groups (Fig. 12B).

T cells from the spinal cord (CNS) could only be sorted from the LO-OPT and HI group. On D5 p.i., the expression of IFN $\gamma$ and IL-17 in the LO-OPT group was strongly upregulated by a factor of almost 3 compared to the HI group (Fig. 12C). On D7 p.i., the expression of both IFN $\gamma$ and IL-17 of the LO-OPT group had decreased again and was similar to the HI group (Fig. 12C). 


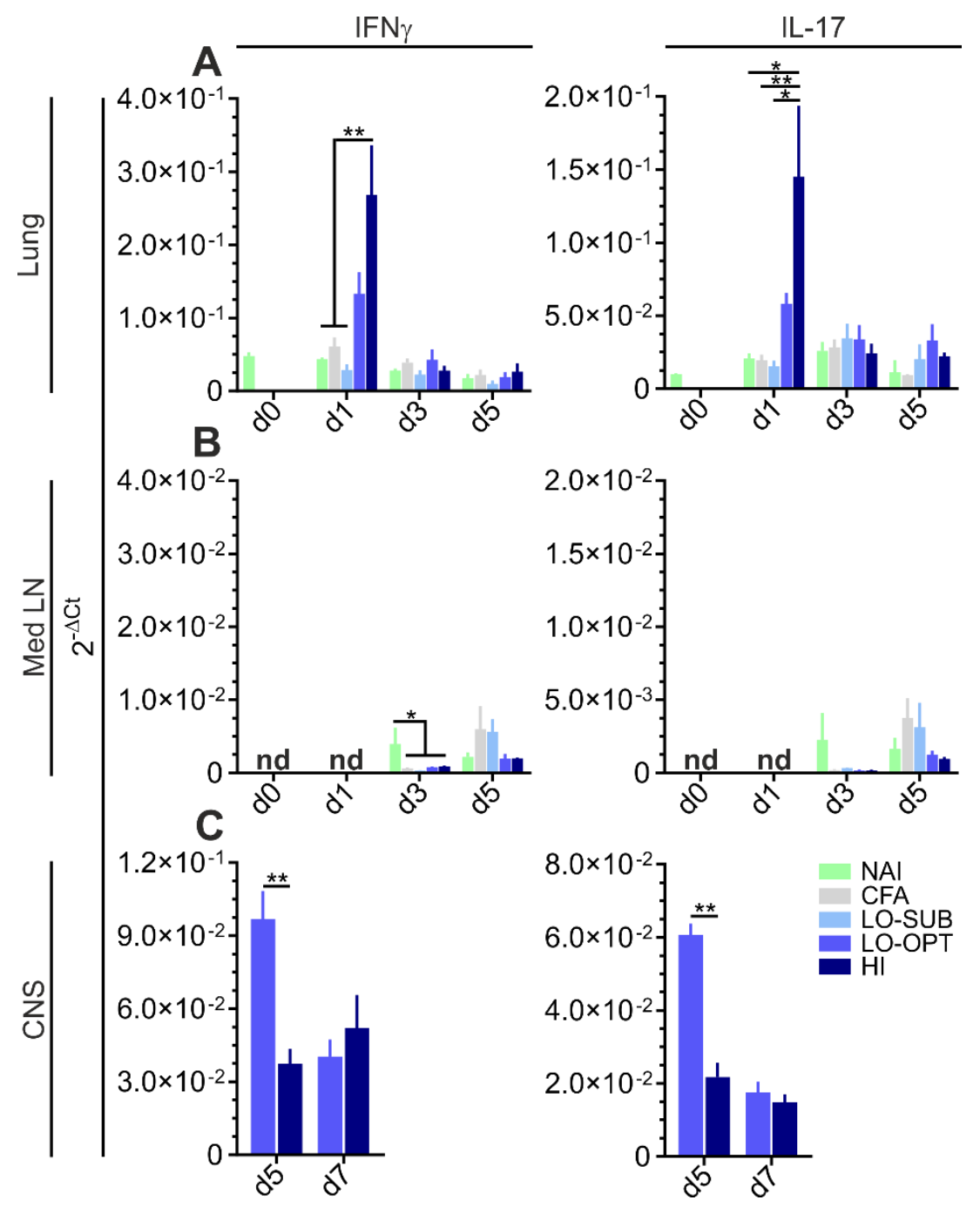

Figure 12: Kinetic of cytokine expression in $\mathrm{T}_{\mathrm{MBP}}$ cells during i.tr. induced EAE. Experimental setup as in Fig. 11. Activation of transferred T cells sorted from lung (A), mediastinal lymph nodes (Med LN; B) and spinal cord (CNS; C) at the indicated time points (days p.i.) was determined on mRNA level using qPCR based on the expression of IFN $\gamma$ (left) and IL-17 (right).

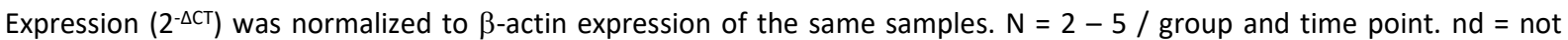
determined. Values are represented as mean \pm SEM. Statistical significance was determined via one-way ANOVA with Tukey's multiple comparisons test (A, B) or unpaired two-tailed $t$-test (C). ${ }^{*} \mathrm{p}<0.05 ;{ }^{*} \mathrm{p}<0.01$.

In addition, we determined the total tissue expression of IFN $\gamma$ and IL-17 in the corresponding lung and CNS samples. Similar to the sorted T cells, the expression of both IFN $\gamma$ and IL-17 tended to by upregulated in the lung tissue of the HI group on D1 p.i., though it was not significantly different from the other groups (Fig. 13A). On D3 p.i., IFN $\gamma$ and IL-17 expression was again similar in all groups (Fig. 13A).

In the CNS, a significant upregulation in IFN $\gamma$ expression and a strong tendency toward a higher expression of IL-17 was observed on D5 p.i in the LO-OPT group compared to the HI group (Fig. 13B). On D7 p.i., the expression of IFN $\gamma$ and IL-17 in the LO-OPT group had decreased again. In contrast, IFN $\gamma$ expression in the CNS of the $\mathrm{HI}$ group tended to be upregulated, though this difference was not significant. IL-17 expression was similar between the LO-OPT and HI group on D7 p.i. (Fig. 13B). 

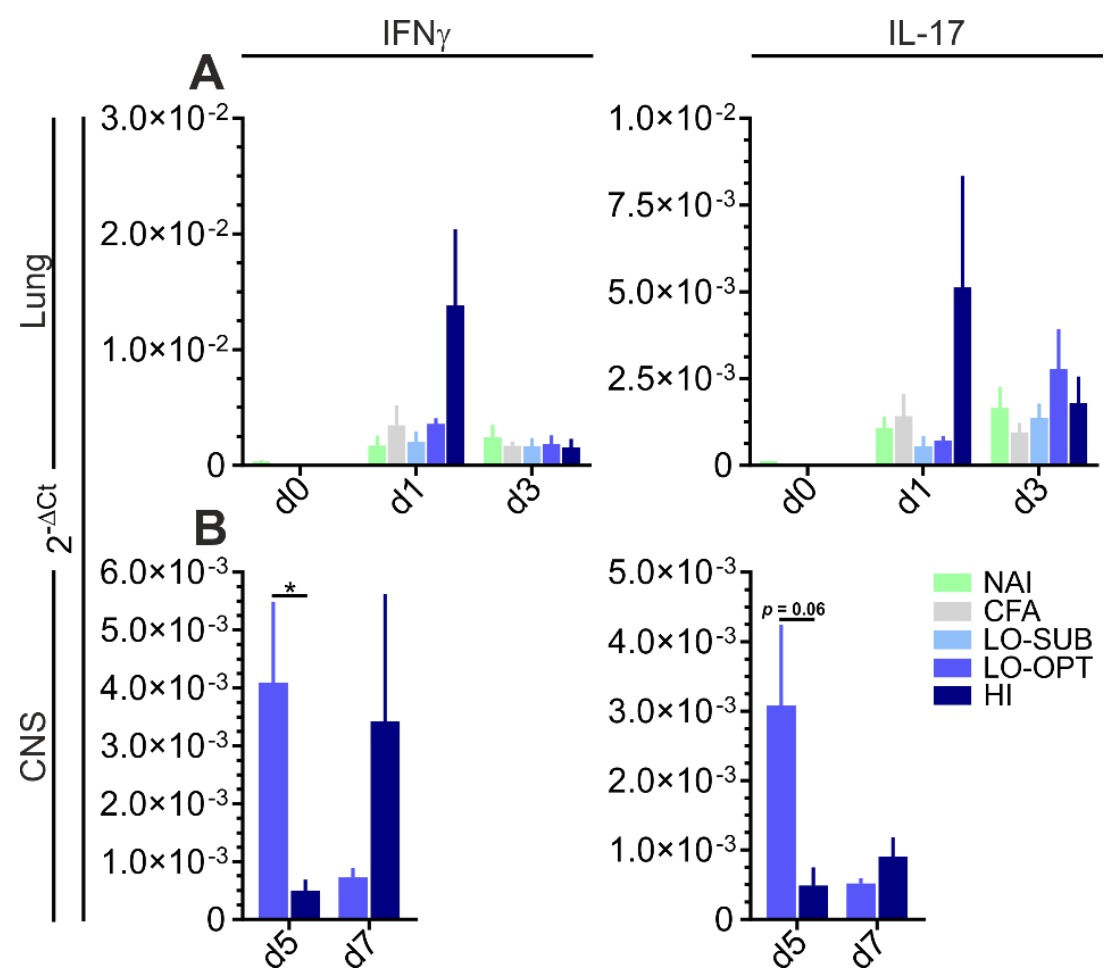

Figure 13: Kinetic of cytokine expression in lung and spinal cord during i.tr. induced EAE. Experimental setup as in Fig. 11. Total tissue expression of IFN $\gamma$ (left) and IL-17 (right) mRNA in lung (A) and spinal cord (CNS; B) at the indicated time points (days p.i.) was determined using qPCR. Expression $\left(2^{-\triangle C T}\right)$ was normalized to $\beta$-actin expression of the same samples. $N=2-$ 5 / group and time point. Values are represented as mean \pm SEM. Statistical significance was determined via one-way ANOVA with Tukey's multiple comparisons test (A) or unpaired two-tailed $t$-test (B). ${ }^{*} p<0.05$.

\subsubsection{Gene expression of $T_{M B P}$ cells is highly regulated during i.tr. induced $E A E$}

In order to achieve an in-depth characterization of the lung EAE model, transcriptome analysis via next-generation sequencing (NGS) was performed on transferred T cells sorted from the lungs before immunization (D0; $12 \mathrm{~h}$ p.t.) and on D1 p.i. (time point of T cell activation), as well as from the blood on D5 p.i. (onset of EAE).

Principle component analysis (PCA) revealed distinct clustering of T cells sorted from lung and blood at each time point, indicating a substantial reprogramming in their global gene expression profile (Fig. 14A). More specifically, of 14,590 genes examined in total, 589 were found regulated in T cells sorted from the lung on D1 p.i. compared to D0. Of these 589 genes, 412 were up- and 177 were downregulated. In the cells sorted from blood on D5 p.i. compared to lung on D1 p.i., 2967 genes were found differently expressed. Among these, 1376 were upand 1591 were downregulated (Fig. 14B).

Of the genes found to be regulated between D0 and D1 p.i. in the lung and between D1 p.i. in lung and D5 p.i. in blood, 388 were regulated in common (Fig. 14C). Interestingly, in this group of shared genes, all genes upregulated on D1 compared to D0 p.i. were downregulated on D5 compared to D1 p.i. and vice versa (Fig. 14D, E), even though these corresponding mutual upand downregulations were of varying extents (Fig. 14D, E). In addition to the shared regulated genes, a large number of genes ( 2579 , i.e. $87 \%$ of the regulated genes) was also found to be regulated independently between blood on D5 p.i. and lung on D1 p.i. (Fig. 14C). 
We next performed biological processes Gene Ontology (GO) term enrichment analysis on the 388 genes commonly regulated genes between D0 and D1 p.i. and between D1 and D5 p.i. The three most significant GO terms were cell division, chromosome segregation and mitotic nuclear division, all of which are crucially involved in the regulation of cell proliferation. Among the significant GO terms were also cellular response to IFN $\gamma$, inflammatory response, chronic inflammatory response and cellular response to TNF, which is in line with the activation of

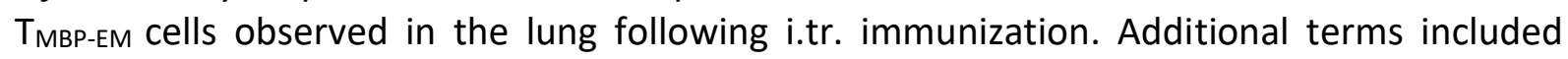
chemotaxis and chemokine-mediated signaling pathway, suggestive of changes in the migratory mode of the transferred T cells (Fig. 14D).

In addition, we performed biological processes GO term enrichment analysis on the 2579 genes exclusively regulated between blood on D5 p.i. and lung on D1 p.i. The two terms most associated with gene expression regulation were positive regulation of telomerase RNA localization to Cajal bodies and positive regulation of protein localization to Cajal bodies, suggestive of changes in assembly and / or processing of ribonucleoprotein complexes. Other terms like DNA replication initiation, DNA-dependent DNA replication, positive regulation of DNA-directed DNA polymerase activity indicated regulation of cell proliferation. Moreover, the GO-term cell-cell adhesion may correlate with an increased CNS-invasive capacity of the T cells (Fig. 14E). Taken together, the transcriptome analysis indicates that i.tr. immunization induced a shift in the gene expression profile of $\mathrm{T}_{\mathrm{MBP}-\mathrm{EM}}$ cells in the lung toward proliferation and activation. In $T$ cells retrieved from the blood after exiting the lung, this profile was partially reversed. In addition, however, a completely different set of genes was differently expressed, suggesting a reprogramming of the T cells on their route to the CNS. 

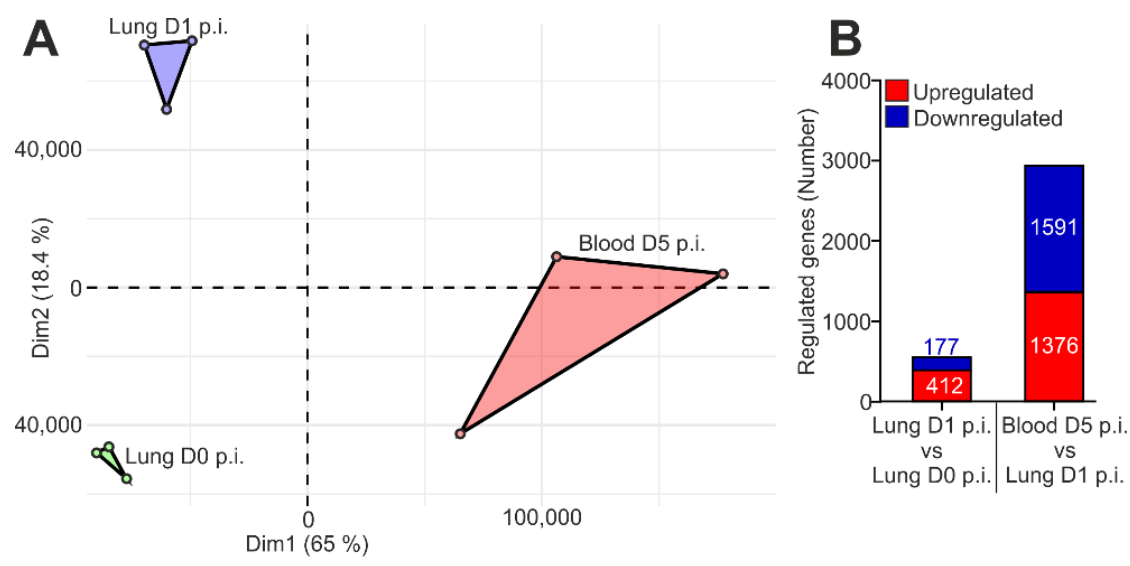

\section{C}

Lung D1 p.i. Blood D5 p.i. $\begin{array}{ccc}\text { vs } & \text { vs } \\ \text { Lung D0 p.i. } & \text { Lung D1 p.i. }\end{array}$

D Blood D5 p.i. Lung D1 p.i.

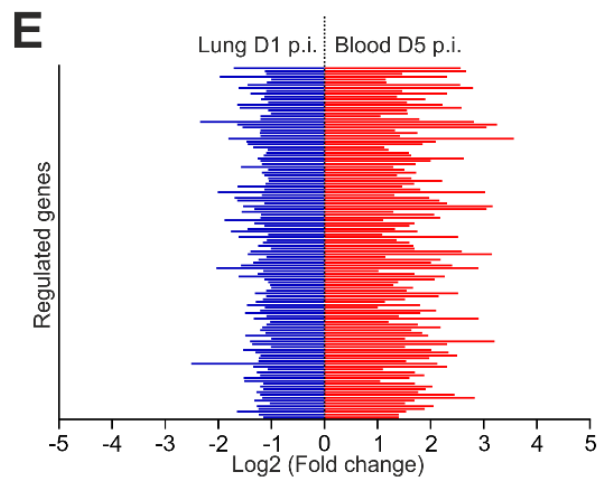

$\mathbf{F}$

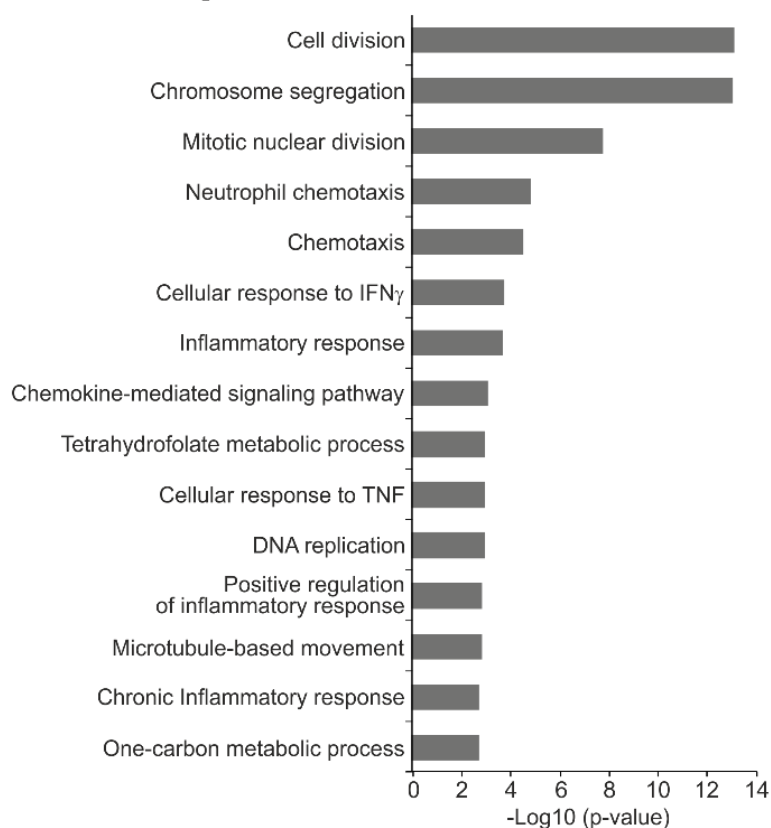

G

Positive regulation of telomerase RNA localization to Cajal body Positive regulation of protein localization to Cajal body

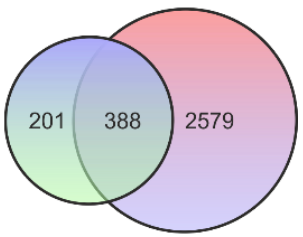

Cell-cell adhesion

Protein folding

TNF-mediated signaling pathway

Cell-redox homeostasis

Toxin transport Positive regulation of establishment of
protein localization to telomere

Small GTPase mediated signal transduction

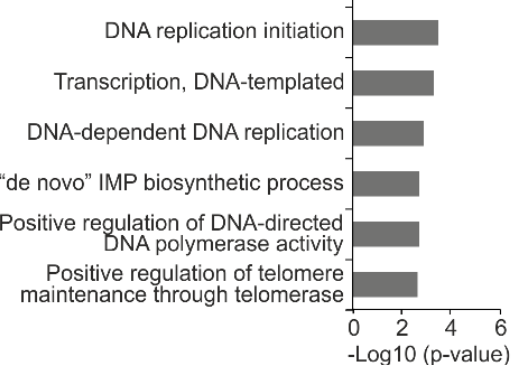

Figure 14: Regulation of the gene expression profile of $T_{M B P}$ cells following i.tr. activation. Rats received $7.5 \times 10^{6} \mathrm{~T}_{\mathrm{MBP}-\mathrm{EM} \text { - }}$ GFP cells and were i.tr. immunized $6-12 \mathrm{~h}$ later under LO-OPT conditions ( $1 \mu \mathrm{g}$ MBP and $10 \mu \mathrm{g}$ CFA / $300 \mathrm{~g}$ body weight). Transferred T cells were sorted from lung on D0 p.i. (12 h p.t.; no immunization) and D1 p.i. and from blood on D5 p.i. Transcriptome analysis was performed using NGS. (A) Principal component analysis (PCA) based on the gene expression of the transferred T cells from the organs and time points indicated. (B) Number of regulated genes between D0 and D1 p.i. in lung (left) and between D1 p.i. in lung and D5 p.i. in blood (right). (C) Number of commonly and independently regulated genes between the indicated time points and organs. (D, E) Fold change (Log2) in the expression of the commonly regulated genes between D0 p.i. and D1 p.i. in lung (Lung D1 p.i.) and between D1 p.i. in lung and D5 p.i. in blood (Blood D5 p.i.). Each individual line represents the fold change in expression of one gene (Log2). Note that the expression of every gene upregulated under one condition is downregulated under the other condition and vice versa. $(F, G)$ Biological processes Gene Ontology (GO) term enrichment analysis of the genes regulated [-Log10 ( $p$-value)] commonly between D0 and D1 p.i. in lung and between D1 p.i. in lung and D5 p.i. in blood (F) or independently between D1 p.i. in lung and D5 p.i. in blood (G). GO term analysis was performed using DAVID. Cumulative data from three independent experiments with $\mathrm{N}=3-4$ / group and time point. 


\subsubsection{Antigen-presenting cells from the lung are capable of activating $T_{M B P-E M}$ cells}

Lung-associated activation of T cells has classically been proposed to be initiated in the lungdraining mediastinal lymph nodes following dendritic cell-mediated antigen uptake in the lung and transport to this location (Sheridan and Lefrançois, 2011; Caucheteux et al., 2013; Lambrecht et al., 2014). In contrast, our experiments demonstrate a strong activation of TMBPEM cells in the lung already $24 \mathrm{~h}$ p.t. Additionally, transferred T cells can barely be detected in the mediastinal lymph nodes before $\mathrm{D} 3 \mathrm{p}$.i.

In order to test the capacity of lung-derived antigen-presenting cells (APCs) to activate $T_{M B P}$ EM cells, we isolated an APC-enriched cell fraction from naïve lungs (Fig. 15A). We determined the immune cell composition of the isolated fraction and observed that most of the isolated cells ( $88 \%$ ) were CD11b/c $\mathrm{c}^{+}$CD172a ${ }^{+}$myeloid cells. Of this fraction, $21.4 \%$ and $78.6 \%$ exhibited a high and low-level expression of $\mathrm{MHCll}$, respectively. The $\mathrm{MHCII}^{\mathrm{LO}}$ myeloid cells could be further sub-categorized in two populations based on their size measured in the forward scatter (FSC): $39 \%$ were relatively small whereas the remaining $61 \%$ exhibited a high FSC intensity, indicating a large cell size, which in combination with a low expression of $\mathrm{MHCll}$ is characteristic of alveolar macrophages. As expected, lymphocytes only constituted a minor proportion of the labelled cells: $8.4 \%$ and $1.2 \%$ were $\mathrm{CD}^{+}$and $\mathrm{CD} 8^{+} \mathrm{T}$ cells, respectively. $\mathrm{B}$ cells made up $2.4 \%$ (Fig. 15B).

Next, we tested the capacity of the isolated lung cell fraction to activate $T_{M B P-E M}$ cells by coculturing both cell populations in presence of MBP. Two days after antigen encounter, $\mathrm{T}$ cells were adoptively transferred into naïve recipient animals (Fig. 15A). All animals developed EAE starting on D4 and peaking on D6 p.t. Afterwards, the clinical scores started to decrease and all animals fully recovered by D11 p.t. (Fig. 15C). 

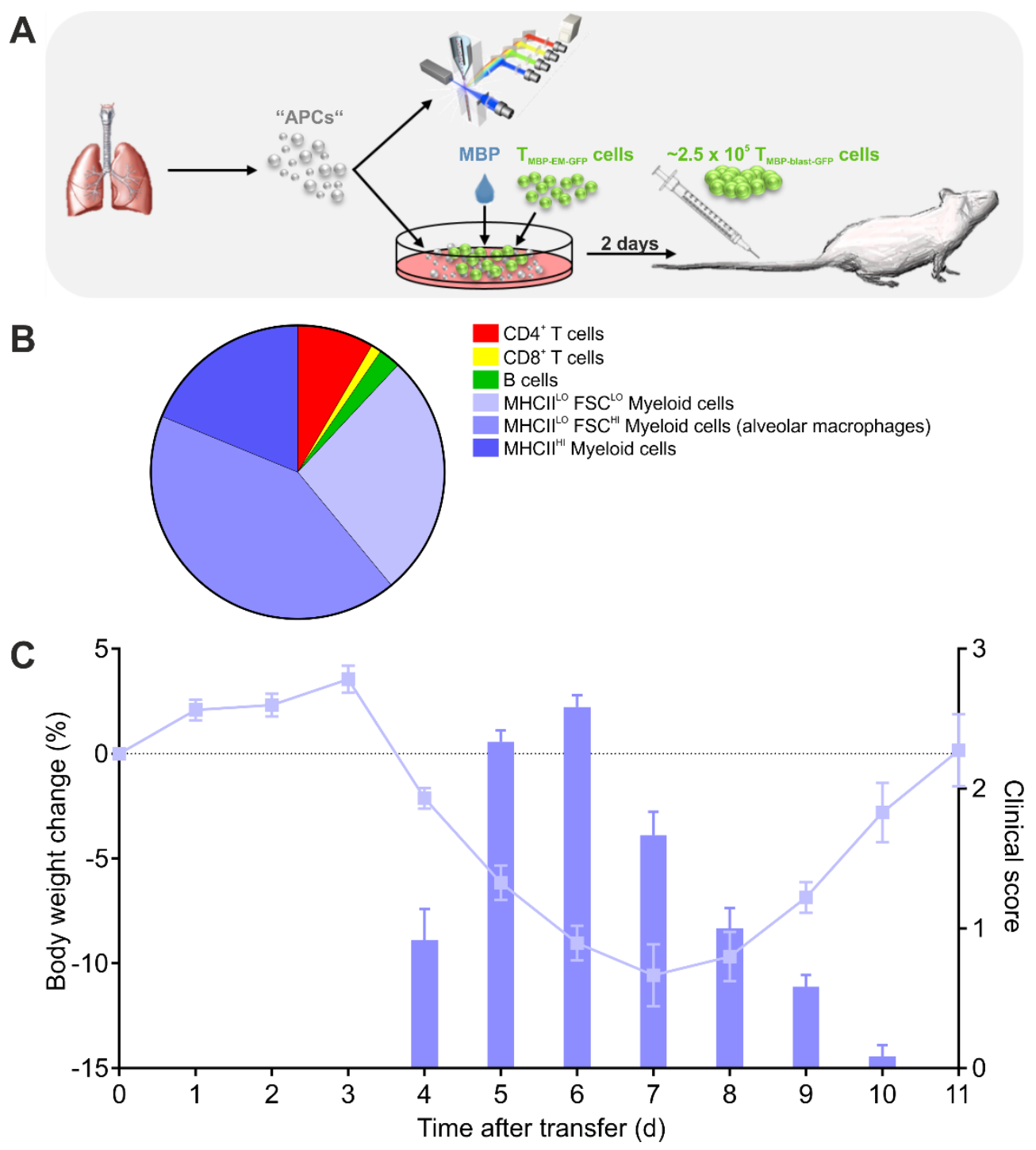

Figure 15: $\mathrm{T}_{\mathrm{MBP}}$ cells stimulated in vitro by lung-derived antigen-presenting cells are encephalitogenic. (A) Experimental design: Lung cells enriched for antigen-presenting cells (APCs) were isolated via a $14.5 \%$ Nycodenz gradient. The immune cells composition of the isolated fraction was determined by surface marker staining and flow cytometry. Moreover, the isolated cells were co-cultured with $\mathrm{T}_{\text {MBP-EM-GFP }}$ cells and MBP. Two days later, $2.5 \times 10^{5} \mathrm{~T}_{\text {MBP-blast-GFP }}$ cells from culture were adoptively transferred into naïve recipient rats. (B) Immune cell composition of the cell fraction isolated from naïve rats. The pie chart represents the percentage of stained cells of the corresponding immune cell populations. The following antibody combinations were used to differentiate immune cell types (\% = percentage of stained cells): $\mathrm{CD}^{+}{ }^{+} \mathrm{T}_{\text {cells: }} \mathrm{CD}^{+} \alpha \beta \mathrm{TCR}^{+}(8.4$

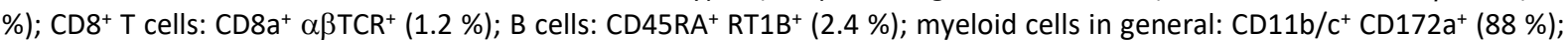
MHCII ${ }^{\mathrm{LO}} \mathrm{FSC}^{\mathrm{LO}}$ myeloid cells (27 \%); MHCII ${ }^{\mathrm{LO}} \mathrm{FSC}^{\mathrm{HI}}$ myeloid cells: alveolar macrophages (42.2\%); MHCII ${ }^{\mathrm{HI}}$ myeloid cells (18.8 $\%)$. (C) Body weight change (lines) and clinical scores (bars) over the course of EAE. $N=3$. 


\subsubsection{Examination of the antigen dose-dependent difference in EAE severity}

\subsubsection{Lung immunization with high antigen doses does not appear to locally trap immune}

cells

While establishing the lung EAE model, we unexpectedly observed that EAE induced by immunization with higher doses of antigen ( $50 \mu \mathrm{g} \mathrm{MBP} \mathrm{/} 300 \mathrm{~g}$ body weight; $\mathrm{HI}$ ) is less severe than when induced using a 50-fold reduced dose (1 $\mu \mathrm{g}$ MBP / $300 \mathrm{~g}$ body weight; LO-OPT; Figs. 7 and 11). We reasoned that a stronger lung inflammation induced by higher doses of antigen could potentially trap innate immune cells within the lung, thereby preventing their contribution to CNS inflammation. In order to test this hypothesis, rats were adoptively transferred with CNS-ignorant TOVA-EM cells and i.tr. immunized with HI or LO-OPT doses of OVA antigen to induce local inflammation. Two days later, the animals additionally received $T_{\text {MBP-blast }}$ cells (Fig. 16A). A control group of animals received TOVA-EM cells and two days later $\mathrm{T}_{\text {MBP-blast }}$ cells without being immunized (NAl; Fig. 16A).

In both the HI OVA and LO-OPT OVA group, EAE onset was delayed by approximately one day compared to the NAI group (Fig 16B, D). Both groups, however, developed EAE of similar severity with identical peak scores of $2.83 \pm 0.08$ (Fig. 16E) and similar cumulative scores (LOOPT OVA: $14.08 \pm 0.72$; HI OVA: $12.92 \pm 0.44)$. The cumulative scores of both groups were significantly lower than of the NAI group ( $21.25 \pm 2.55$; Fig. 16F). Taken together, these data suggest a mild but antigen dose-independent effect of i.tr. immunization on the clinical outcome of EAE that obviously cannot justify the observed difference in clinical outcome following i.tr. immunization with different doses of MBP. 
A

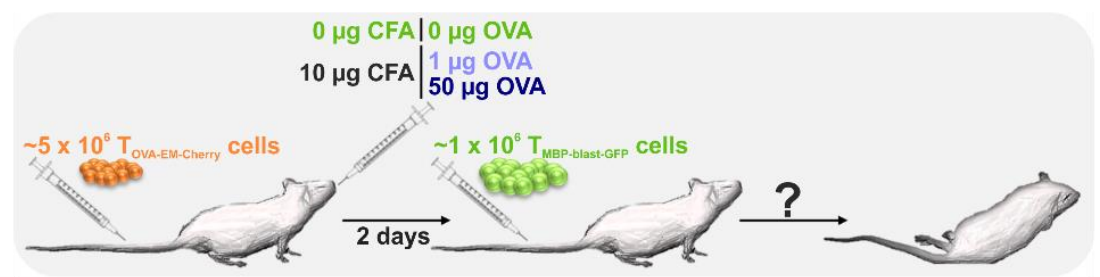

B
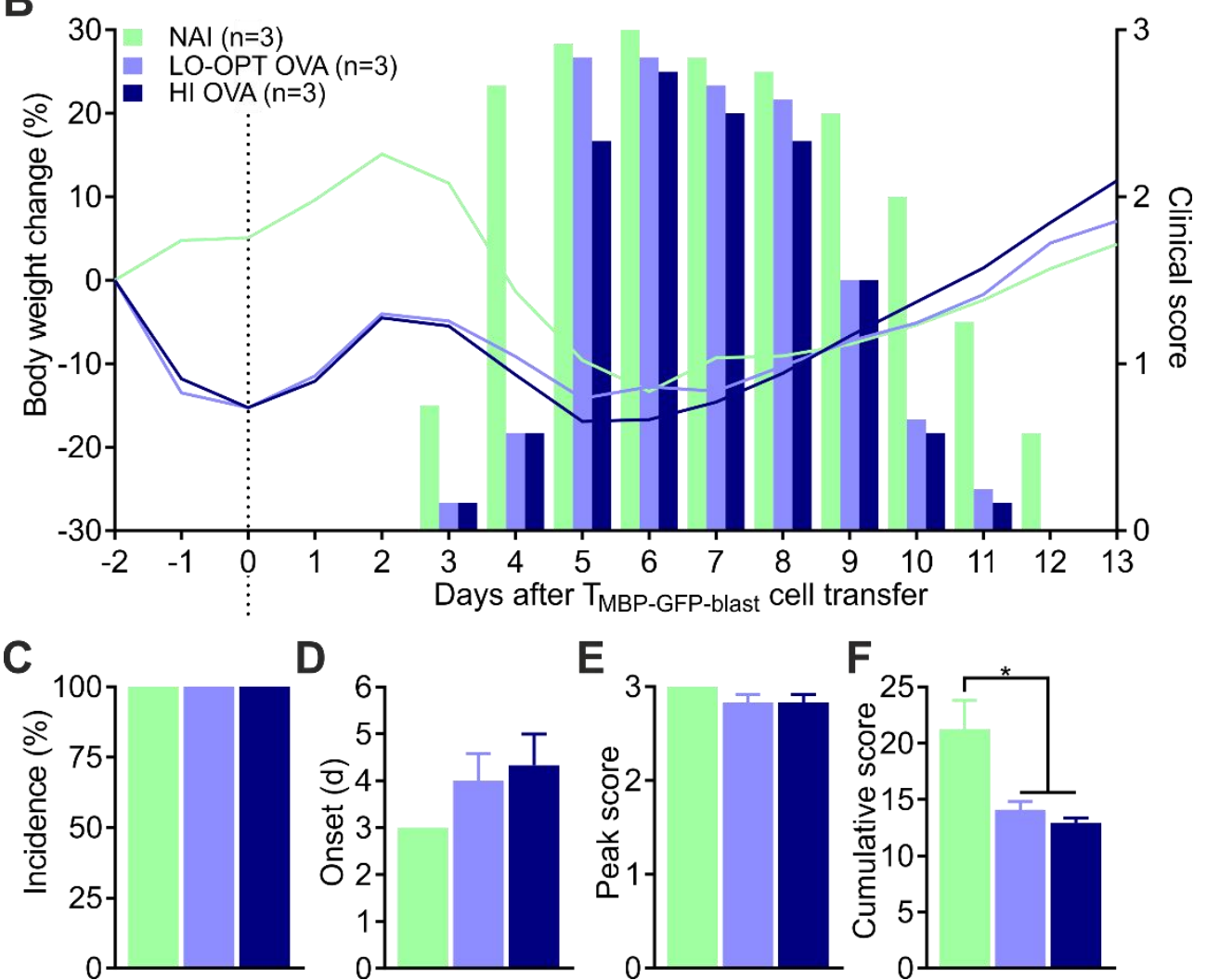

E
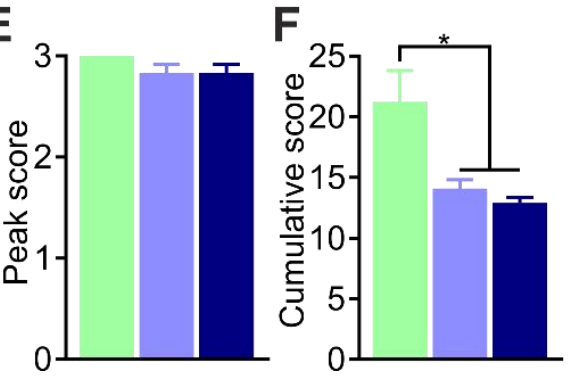

Figure 16: $\mathrm{T}_{\mathrm{MBP} \text {-blast }}$ cell transfer following i.tr. immunization with high or low concentrations of a non-self antigen. (A) Experimental design: Rats received $5 \times 10^{6}$ ToVA-EM-Cherry cells i.v. and were i.tr. immunized $6-12 \mathrm{~h}$ later with a high $(50 \mu \mathrm{g} /$ $300 \mathrm{~g}$ body weight; HI) or low ( $1 \mu \mathrm{g} / 300 \mathrm{~g}$ body weight; LO-OPT) dose of OVA and CFA (10 $\mu \mathrm{g} / 300 \mathrm{~g}$ body weight). A control group received cells but was not immunized (NAI). Two days later, the animals were transferred with $1 \times 10^{6}$ in vitro activated

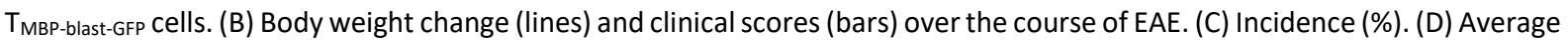
onset (days p.i.). (E) Average peak score. (F) Average cumulative score. Values are represented as mean \pm SEM. Statistical significance was determined via one-way ANOVA with Tukey's multiple comparisons test. ${ }^{*} p<0.05$.

\subsubsection{2 $\mathrm{T}_{\text {MBP-EM }}$ cells activated in the lung with high antigen concentrations do not appear to be anergic or impaired in their proliferation potential}

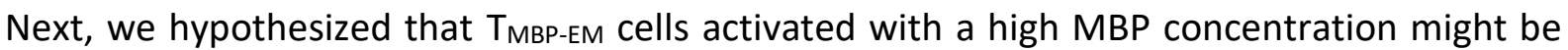
impaired in their functionality: indeed, strong TCR stimulation can induce T cell exhaustion or anergy. To address this hypothesis, rats were i.tr. immunized with the HI or LO-OPT MBP dose following $T_{M B P-E M}$ cell transfer. After 7 days, the transferred $T$ cells were isolated from peripheral organs and stimulated in vitro (Fig. 17A). T cell activation and proliferation served as read-out (Fig. 17B, C).

Compared to the control conditions (no antigen; -MBP), T cells isolated from both the $\mathrm{HI}$ and the LO-OPT group strongly increased the expression of IFN $\gamma$ and IL-17 mRNA, indicative of an 
ongoing activation. However, no significant differences in cytokine expression could be observed between the antigen-stimulated (+MBP) groups. (Fig. 17B).

A slightly higher expression of the surface activation marker IL-2R $\alpha$ (CD25) was observed in T cells from the LO-OPT group (Fig. 17C) upon antigen stimulation. However, the expression in both the LO-OPT +MBP and the HI +MBP group was highly upregulated compared to the corresponding -MBP control groups.

The proliferation of T cells from both groups was similar on D2 after antigen encounter and further increased on D3 and D4 following the addition of IL-2 in both groups.

Taken together and despite minor differences in activation and proliferation between the groups, these data indicate that $T$ cells activated in the lung using high antigen concentrations were not impaired in their basic functions.

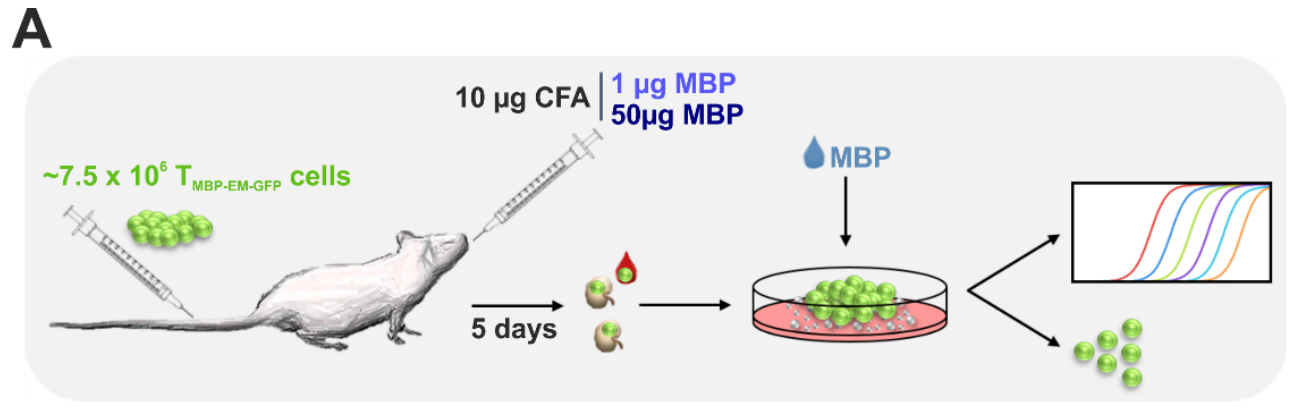

\section{B}
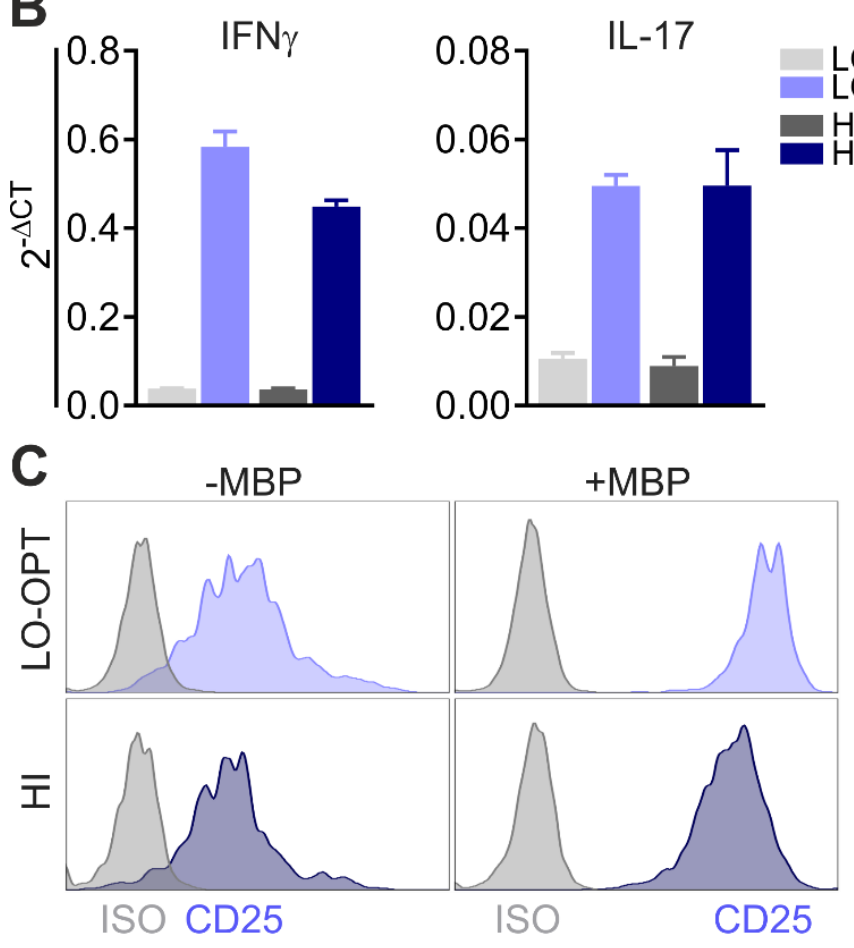

O-OPT / -MBP / -MBP $\mathrm{HI} /+\mathrm{MBP}$

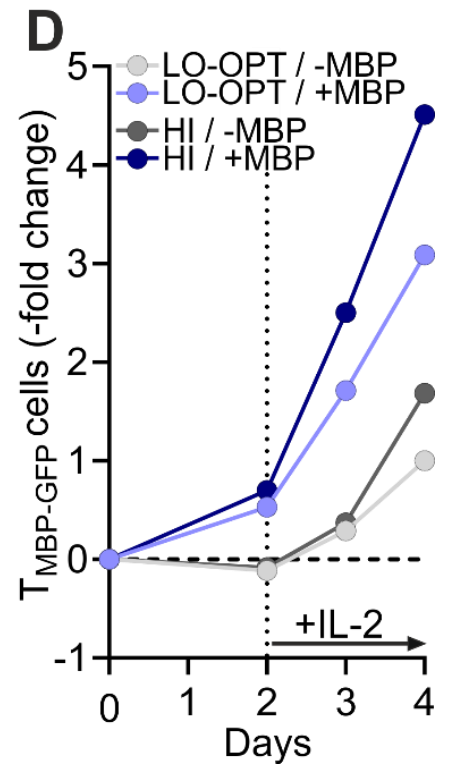

Figure 17: Proliferation and activation of $\mathrm{T}_{\mathrm{MBP}}$ cells previously activated in the lung with high or low MBP concentrations. (A) Experimental design: Rats received $7.5 \times 10^{6} \mathrm{~T}_{\text {MBP-EM-GFP }}$ cells i.v. and were i.tr. immunized $6-12 \mathrm{~h}$ later with a high (50 $\mu \mathrm{g}$

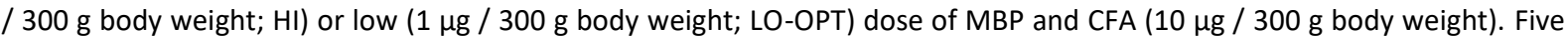
days later, the transferred T cells were isolated from blood and mediastinal lymph nodes and stimulated with $(+)$ or without (-) MBP and irradiated thymocytes in vitro. T cell activation and proliferation was assessed via qPCR and flow cytometry. (B)

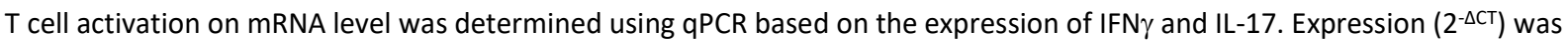
normalized to $\beta$-actin expression of the same samples. (C) T cell activation on protein level was determined using antibody labeling and flow cytometry based on the expression of CD25. (D) Changes in T cell numbers on D2, 3 and 4 compared to D0 were determined using flow cytometry. On D2, IL-2 was added to the cells to boost proliferation. Representative data of two independent experiments. 


\subsubsection{The antigen dose-dependent difference in EAE severity is T cell-intrinsic and transferable}

Next, we examined whether the observed difference in EAE severity between the $\mathrm{HI}$ and the LO-OPT group was T cell-intrinsic. To this end, rats were i.tr. immunized with the HI or LO-OPT MBP dose following $T_{\text {MBP-EM }}$ cell transfer. After 3.5 days, the spleens were harvested and the transferred T cells isolated. Equal numbers of previously transferred TMBP-EM cells isolated from the spleens of both donor groups were subsequently re-transferred into naïve recipient animals ( $\mathrm{HI}$ and LO-OPT recipient group). After additional 3.5 days, the re-transferred T cells were quantified in different organs of the recipients (Fig. 18A).

In all peripheral organs examined, the amount of T cells was similar between the groups with slight tendencies toward a higher number in the lung of the LO-OPT recipient group and in the blood of the $\mathrm{HI}$ recipient group (Fig. 18B). In contrast, in the spinal cord, a significant difference in T cell numbers could be observed between the groups: an average of $1.13 \times 10^{5} \mathrm{~T}$ cells / $\mathrm{g}$ was detected in the LO-OPT recipient group compared to an average of $2.26 \times 10^{4}$ cells $/ \mathrm{g}$ in the spinal cord of the $\mathrm{HI}$ recipient group (Fig. 18B). Owing to the generally low yield of retransferrable T cells and comparably low number of these cells entering the CNS of the retransfer recipient animals, only mild clinical symptoms occurred. Importantly, this was only the case in the recipient animals of the T cells isolated from the LO-OPT group. The HI recipient group, in contrast, remained symptom-free (data not shown). Consequently, the dosedependent difference in EAE severity appears to be T cell-intrinsic.

A
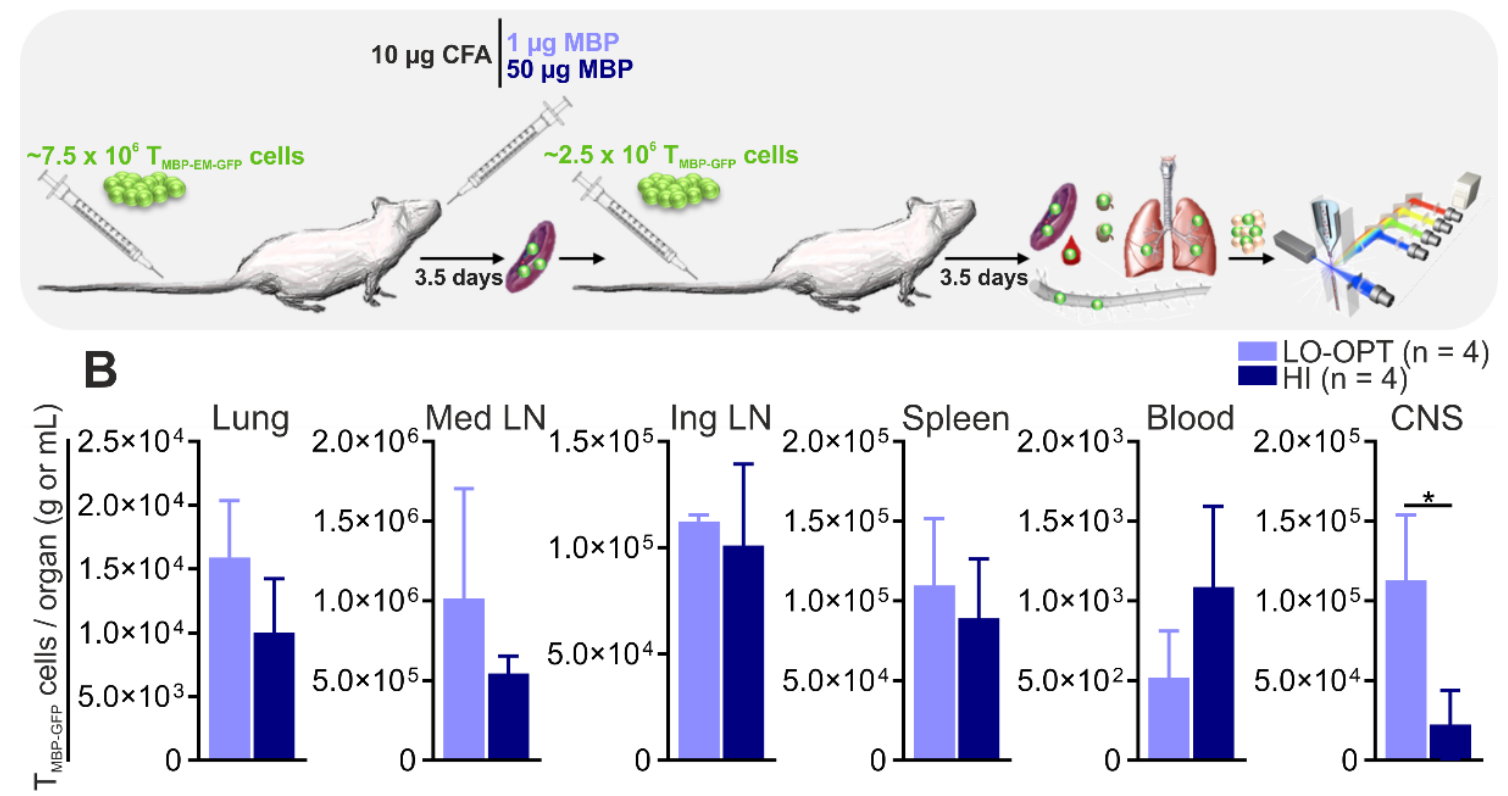

Figure 18: Re-transfer of $\mathrm{T}_{\mathrm{MBP}}$ cells from rats i.tr. immunized with high or low MBP concentrations. (A) Experimental design:

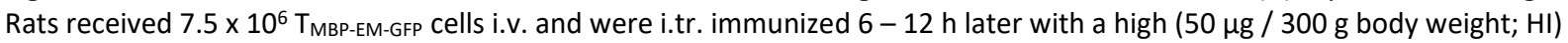
or low (1 $\mathrm{\mu g} / 300 \mathrm{~g}$ body weight; LO-OPT) dose of MBP and CFA (10 $\mu \mathrm{g} / 300 \mathrm{~g}$ body weight). 3.5 days later, the transferred $\mathrm{T}$ cells were isolated from the spleens of both groups. $2.5 \times 10^{6}$ isolated T cells were re-transferred into naïve recipient animals. Another 3.5 days later, $\mathrm{T}$ cell distribution in different organs was determined via flow cytometry. (B) T cells per g or $\mathrm{mL}$ detected in the indicated organs. Cumulative data from four independent experiments. Values are represented as mean \pm SEM. Statistical significance was determined via two-tailed $t$-test. $* p<0.05$. 


\subsubsection{Similar ratios of TCR-V $\beta$-T cell subclones in blood and spinal cord of the $\mathrm{HI}$ and the LO-OPT group}

The $T_{\text {MBP-EM }}$ cell lines used in our setup are established in WT Lewis rats and therefore polyclonal, meaning that they potentially could express differing T cell receptors (TCRs) with distinct capacities to react to MBP epitopes. Consequently, they could exhibit varying encephalitogenic potentials. A restricted amount of available antigen could favor the activation and expansion of potentially more pathogenic TMBP-EM cell subclones carrying a TCR with an optimal affinity / avidity for a specific MBP epitope (Fig. 19A).

In order to test whether this hypothesis might explain the effect underlying the observed antigen dose-dependent difference in EAE severity, rats were i.tr. immunized with the $\mathrm{HI}$ or LO-OPT MBP dose following $\mathrm{T}_{\text {MBP-EM }}$ cell transfer. 5 days later, the percentage of different $\mathrm{T}$ cell subclones in the blood and CNS of the two groups was determined based on their expression of the common MBP-specific V $\beta$-chains 16, 10, 8.5 and 8.2 (Fig. 19B).

In the blood, the percentage of the different TCR-V $\beta$-chain ${ }^{+} \mathrm{T}$ cells was not distinguishable between the two groups. Indeed, more than $80 \%$ of the labelled $\mathrm{T}$ cells in both groups expressed the TCR-V $\beta$-chain variant 8.2, (Fig. 19C, D). 6 - 7 \% expressed the TCR-V $\beta 16$ chain and $4-5 \%$ of the T cells was positively labelled for either TCR-V $\beta 8.5$ or V $\beta 10$ (Fig. 19C).

In the spinal cord, the expression pattern of the different TCR was similar between the groups but the percentage differed slightly: $80 \%$ of the cells in the spinal cord of the HI group expressed the TCR-V $\beta 8.2$ chain. In the LO-OPT group, this percentage was decreased to $55 \%$. A significant difference between the groups was observed for the expression of the TCR-V $\beta 16$ chain: about $18 \%$ of the transferred T cells of the LO-OPT group were positively labelled for this variant, compared to $8.6 \%$ in the HI group. A similar trend (17\% compared to $7 \%$ ) but without reaching statistical significance could be observed for the TCR-V $\beta 10^{+} \mathrm{T}$ cells. With 6 and $10 \%$ of labelled cells, the TCR-V $\beta 8.5^{+} \mathrm{T}$ cell ratios were more similar between the $\mathrm{HI}$ and LO-OPT group, respectively (Fig. 19D). Given that there was no difference detectable in the ratio of TCR-V $\beta^{+}$T cells in the blood, a selective expansion of a particularly pathogenic $T_{M B P-E M}$ cell subclone seems unlikely but cannot be excluded. 
A

Pathogenicity or
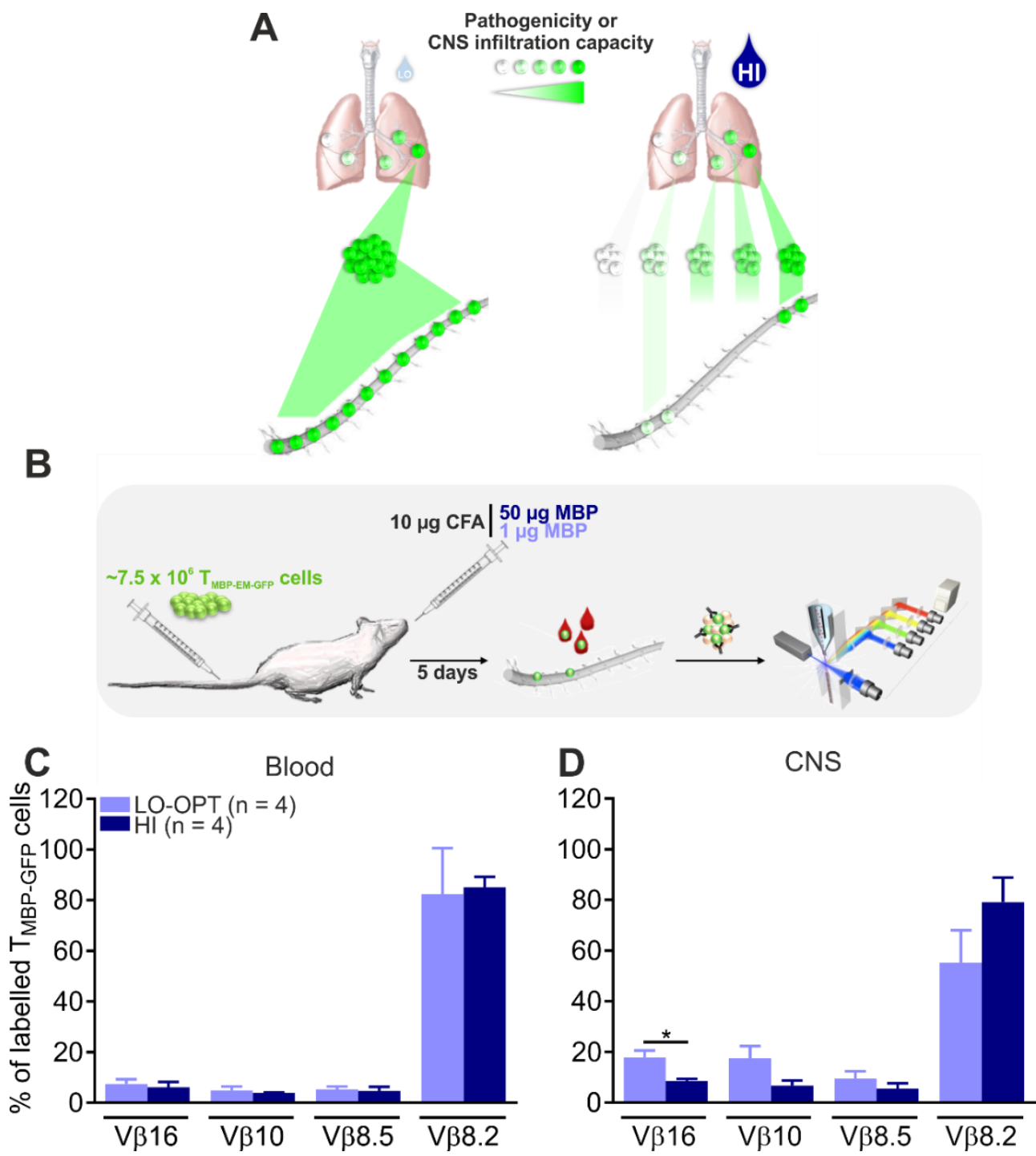

Figure 19: Ratio of different TCR-V $\beta$-T cell subclones in blood and CNS of rats i.tr. immunized with high or low MBP concentrations. (A) Hypothesis: The antigen dose-dependent difference in EAE severity could result from a differential $T_{M B P}$ EM cell subclone expansion. Low concentrations of MBP might result in the selective expansion of subclones that are more pathogenic or more capable of transgressing the BBB. (B) Experimental design: Rats received $7.5 \times 10^{6} \mathrm{~T}_{\text {MBP-EM-GFP cells i.v. and }}$ were i.tr. immunized $6-12 \mathrm{~h}$ later with a high $(50 \mu \mathrm{g} / 300 \mathrm{~g}$ body weight; $\mathrm{HI})$ or low ( $1 \mu \mathrm{g} / 300 \mathrm{~g}$ body weight; LO-OPT) dose of MBP and CFA (10 $\mu \mathrm{g} / 300 \mathrm{~g}$ body weight). Five days later, the transferred T cells in blood and spinal cord (CNS) were antibody-labeled for the expression of different TCR-V $\beta$ chains (V $\beta 16 ; \mathrm{V} \beta 10 ; \mathrm{V} \beta 8.5 ; \mathrm{V} \beta 8.2)$ and acquired via flow cytometry. $(C-D)$ Percentage of the different antibody-labeled TCR-V $\beta$ - $T_{\text {MBP-GFP }}$ cell subclones in the blood (C) and spinal cord (CNS; D). Cumulative data from two independent experiments. Values are represented as mean \pm SEM. Statistical significance was determined via two-tailed $t$-test. ${ }^{*} \mathrm{p}<0.05$. 


\subsubsection{The antigen dose-dependent difference in EAE severity still occurs when using a monoclonal $T_{M B P-E M}$ cell line}

To definitely address whether a selective expansion of a specific TCR subclone could underlay the observed difference in EAE severity using LO-OPT or HI antigen doses, we took advantage

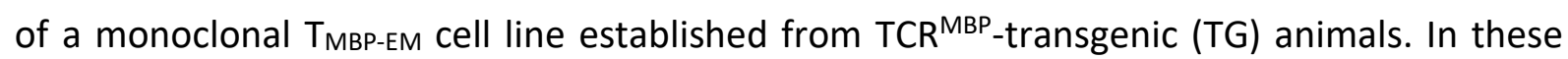
animals, about $95 \%$ of $\alpha \beta T C R^{+} T$ cells express the identical TCR-V $\beta$-chain variant 8.2. Of note, this $\mathrm{TCR}^{\mathrm{MBP}}$ reacts in vitro to antigen doses around 100 times lower than commonly used for WT T cells.

Rats were adoptively transferred with TG $\mathrm{T}_{\mathrm{MBP}-\mathrm{EM}}$ cells and i.tr. immunized with the following

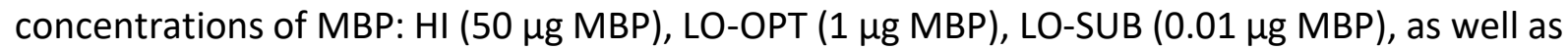
an extremely low antigen dose (XTR-LO; $0.1 \mathrm{ng} \mathrm{MBP}$; all concentrations per $300 \mathrm{~g}$ body weight). Animals transferred with TG T cells but not immunized (NAI) or immunized with CFA alone (CFA) served as control groups.

Similar to the WT cell line, TG T cells induced EAE of varying severity dependent on the antigen concentration used for their activation. As observed using WT cells, the most severe EAE with an incidence of $100 \%$ and a peak score of $2.16 \pm 0.32$ was induced in the LO-OPT group. The $\mathrm{HI}$ group only developed a mild EAE with an incidence of $62.5 \%$ and a peak score of $0.78 \pm$ 0.3. As expected, EAE induced in the LO-SUB group was stronger compared to using WT cells. The incidence was $85.7 \%$ and the average peak score was $1.4 \pm 0.42$ (Fig. 20A - D). Onset in all three groups was between D5 and 5.4 p.i. (Fig. 20C). The NAI, CFA and XTR-LO groups remained disease-free throughout the course of the experiment.

In addition to the clinical course, we determined the distribution of TG T cells in different organs on D5 p.i., that is, between onset and peak of the disease. With minor exceptions in lung and inguinal lymph nodes, the cell numbers were comparable in all peripheral organs examined (Fig. 20F - J). In contrast, clear differences in TG T cell numbers between the groups were observed in the spinal cord: in line with the clinical course, the by far and significantly highest number of TG T cells compared to all other groups $\left(3.73 \times 10^{5} / \mathrm{g}\right)$ was detected in the LO-OPT group. In the HI group, TG T cell numbers were about $10 x$ lower $\left(3.33 \times 10^{4} / \mathrm{g}\right)$. In the LO-SUB group, $7.9 \times 10^{4}$ TG T cells / g had infiltrated the spinal cord by D5 p.i. The XTR-LO group showed low-level TG T cell infiltration of about $2.5 \times 10^{4}$ cells / g. No cells were detected in the spinal cord of the CFA and the NAI group (Fig. 20K).

The fact that the antigen dose-dependent difference in EAE could also be observed when using a TCR-transgenic monoclonal $T_{M B P-E M}$ cell line demonstrates that a differential and selective $T$ cell subclone expansion when immunizing with low antigen concentrations can be excluded as a potential underlying mechanism. 
A
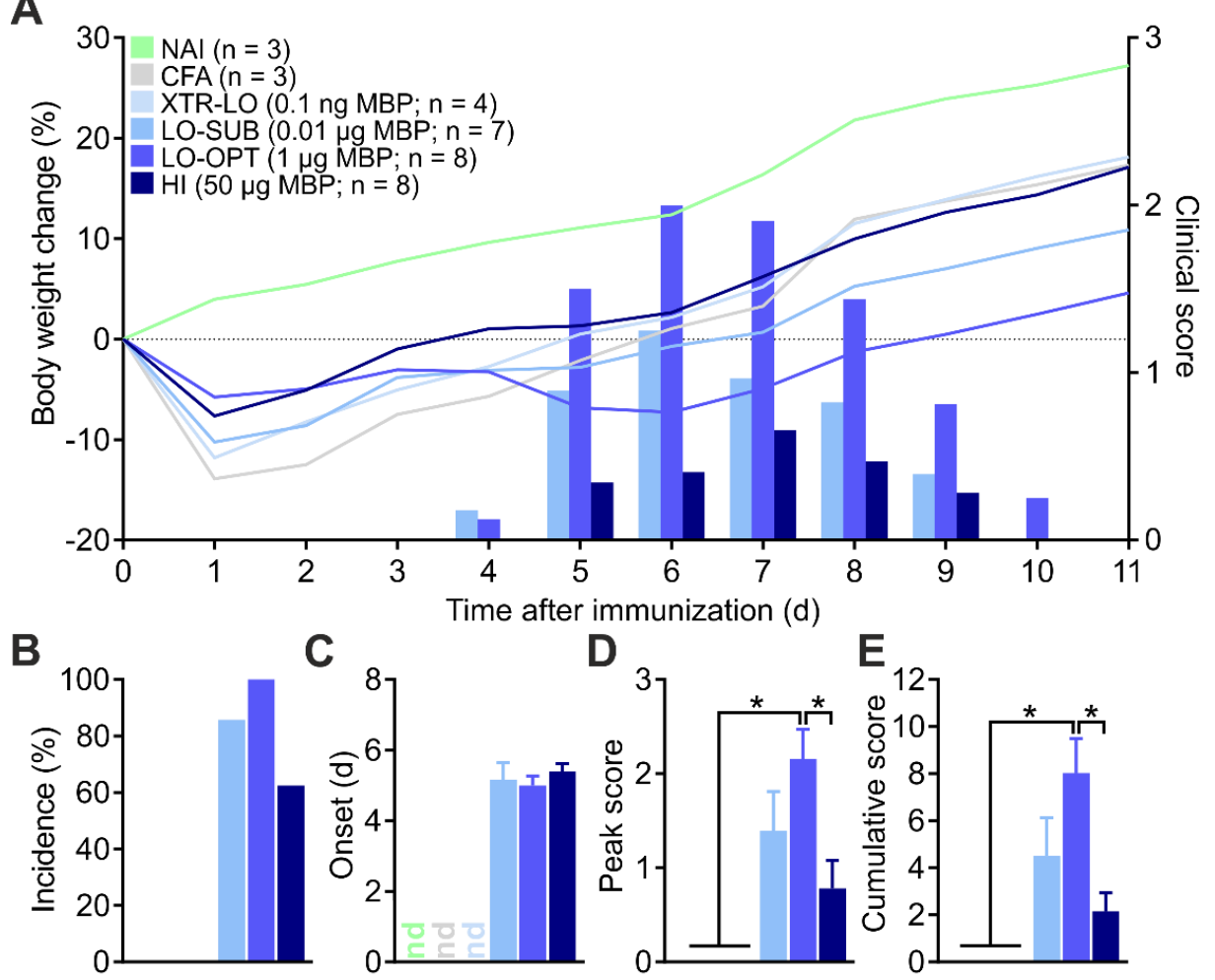

C
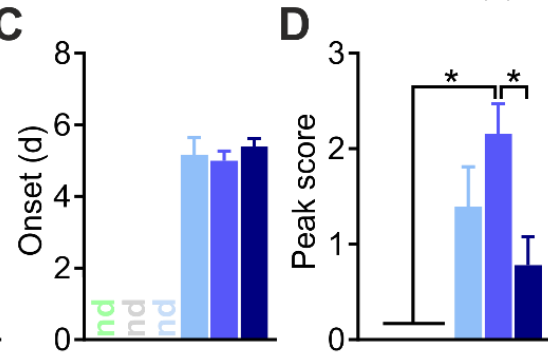

$\mathrm{E}$

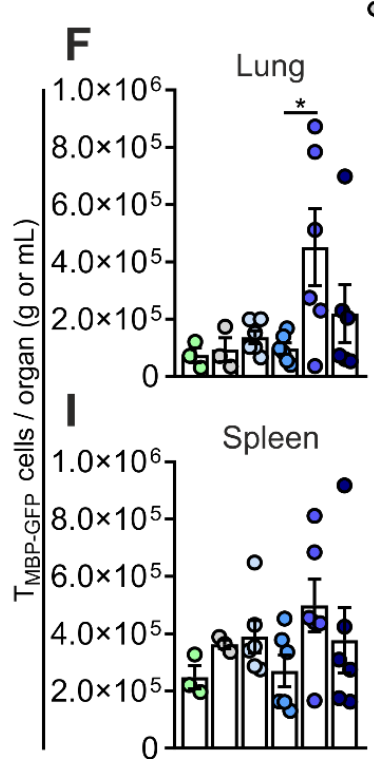

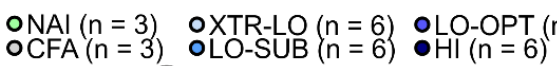

G

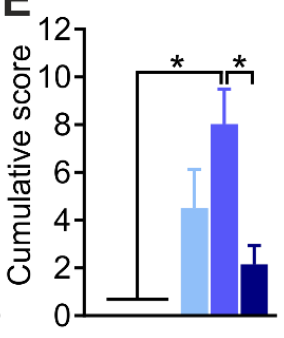

$\mathrm{H}$
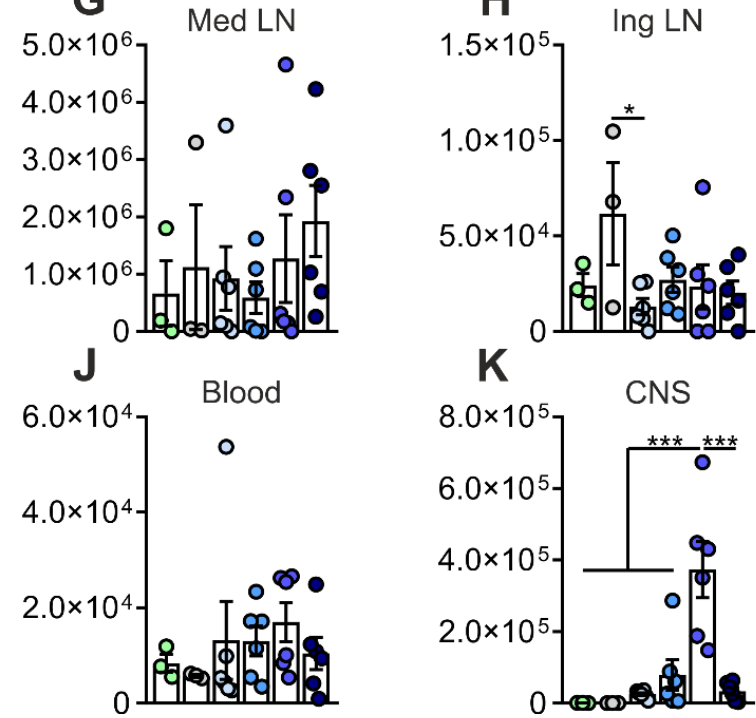

Figure 20: Characterization of TCR ${ }^{\text {MBP-transgenic }}$ T cell distribution in different organs during i.tr. induced EAE. Rats received $7.5 \times 10^{6} \mathrm{TCR}^{\mathrm{MBP}}$-transgenic (TG) $\mathrm{T}_{\mathrm{MBP} \text {-EM-GFP }}$ cells and were i.tr. immunized $6-12 \mathrm{~h}$ later with varying doses of MBP $(0 \mu \mathrm{gg}$ : NAI

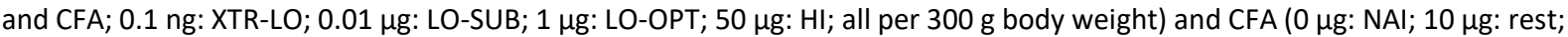
all per $300 \mathrm{~g}$ body weight). (A) Body weight change (lines) and clinical scores (bars) over the course of EAE. (B) Incidence (\%). (C) Average onset (days p.i.). (D) Average peak score. (E) Average cumulative score. ( $F-K) T G$ T cells per $g$ or $\mathrm{mL}$ detected in the indicated organs on D5 p.i. Cumulative data from three (A) or two (B - K) independent experiments. Values are represented as mean \pm SEM. Statistical significance was determined via one-way ANOVA with Tukey's multiple comparisons test. $* p<0.05 ; * * p<0.001$. 


\subsubsection{The expression of adhesion-related genes by $T_{M B P}$ cells activated in the lung may be differently regulated by different antigen concentrations}

Next, we investigated if $\mathrm{T}_{\mathrm{MBP}-\mathrm{EM}}$ cells activated in the lung with higher antigen concentrations might be impaired in their potential to transmigrate through the BBB and enter the CNS.

To test this hypothesis, we determined the expression of integrins and chemokine-receptors known to be relevant for T cell infiltration into the CNS in transferred T cells sorted on D5 p.i. (onset of EAE) from the blood of animals immunized with varying MBP concentrations (LOSUB, LO-OPT, HI), only CFA (CFA), or not immunized at all (NAl; Figs. $10-12$ ).

VLA-4 expression of T cells from both the $\mathrm{HI}$ and LO-OPT group was about twice as high as in the NAl group. This increased expression, however, was similar in both groups. No upregulation of VLA-4 could be observed in the CFA and the LO-SUB group (Fig. 21A).

LFA-1 expression of T cells from the blood of the HI group was significantly reduced by almost $50 \%$ compared to the NAI group. Furthermore, it was significantly lower than in the CFA and LO-SUB but not the LO-OPT group (Fig. 21B). No significant difference could be observed between the NAI, CFA, LO-SUB and LO-OPT groups.

The expression of CXCR3 tended to be upregulated in all groups compared to the NAI group. The only significant difference, however, was observed between the NAI and LO-OPT group. CXCR4 was significantly downregulated in T cells from the blood of the CFA, LO-OPT and HI groups compared to the NAI group. The difference between NAI and $\mathrm{HI}$ was most pronounced: CXCR4 expression in the $\mathrm{HI}$ group was decreased by more than $70 \%$ (Fig. 21B). Downregulation of CXCR4 expression in the CFA and the LO-OPT group compared to the NAI group was about 33 and $40 \%$, respectively (Fig. 21B).

No difference in the expression of CCR5 and CCR6 could be observed between the groups (Fig. 21B).

Taken together, we could observe some differences between the groups in the expression of adhesion-related genes. In case of LFA-1 and CXCR4, the expression was most strongly downregulated in the $\mathrm{HI}$ group. Whether these effects may be of relevance regarding the differences observed in EAE severity remains to be further examined. 

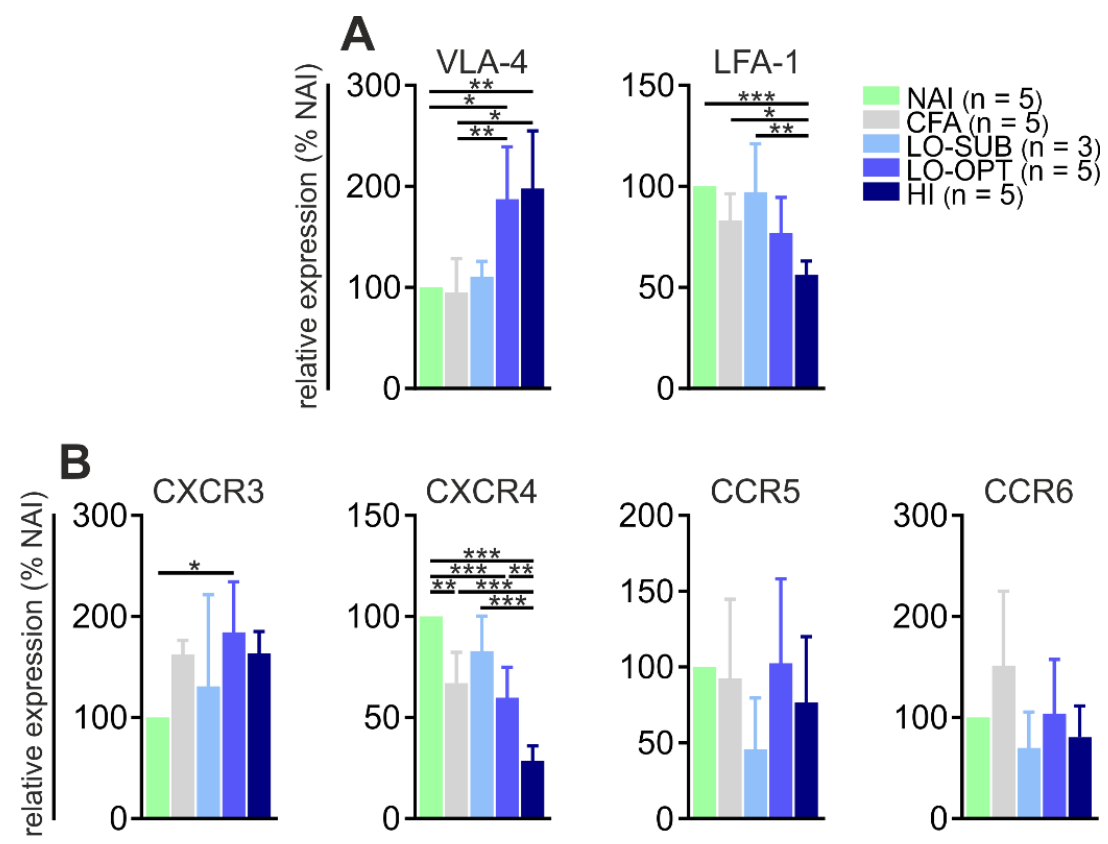

Figure 21: Antigen dose-dependent integrin- and chemokine-receptor expression of $\mathrm{T}_{\mathrm{MBP}}$ cells from blood before CNS infiltration. Rats received $7.5 \times 10^{6} \mathrm{~T}_{\mathrm{MBP}-\mathrm{EM} \text {-GFP }}$ cells and were i.tr. immunized $6-12 \mathrm{~h}$ later with varying doses of MBP $(0 \mu \mathrm{g}$ : $\mathrm{NAI}$ and CFA; $0.01 \mu \mathrm{g}$ : LO-SUB; $1 \mu \mathrm{g}$ : LO-OPT; $50 \mu \mathrm{g}$ : HI; all per $300 \mathrm{~g}$ body weight) and CFA (0 $\mu \mathrm{g}: \mathrm{NAl} ; 10 \mu \mathrm{g}$ : rest; all per 300 $\mathrm{g}$ body weight). On D5 p.i., the transferred T cells were sorted from the blood and the expression of different integrins and chemokine-receptors was determined using qPCR. (A) Relative expression (difference compared to NAl in \%) of the integrins VLA-4 (left) and LFA-1 (right). (B) Relative expression (difference compared to NAI in \%) of the chemokine-receptors CXCR3, CXCR4, CCR5, CCR6 (from left to right). Expression of all genes $\left(2^{-\triangle C T}\right)$ was normalized to $\beta$-actin expression of the same samples. Cumulative data from three independent experiments. $\mathrm{N}=3-5$ / group. Values are represented as mean \pm SEM. Statistical significance was determined via one-way ANOVA with Tukey's multiple comparisons test. ${ }^{*} p<0.05 ;{ }^{* *} p<0.01$; $* * * p<0.001$. 


\subsection{The role of lung and gut microbiota for i.tr. induced $E A E$}

\subsubsection{The lung hosts a complex microbiota distinct from the gut microbiota}

First, we aimed to characterize the lung and gut microbiota of the Lewis rats used in our experiments. To this end, BAL fluid (BALF) and feces were collected from a group of animals. The animals had been i.tr. treated with sterile PBS for 7 days as they served as control group for another experimental setup (see 3.2.4). DNA from the samples was purified and analyzed via NGS to characterize the bacterial communities. The V3 - V4 16S rRNA region was used to determine the operational taxonomic units (OTUs) and exact amplicon sequence variants (ASVs). For the BALF samples, a total of 2,062 ASVs were identified from 950,104 reads. In comparison, a total of 4,185 ASVs were identified from 742,446 reads in the fecal samples.

The most dominant phyla found in the BALF samples were Firmicutes ( $28.3 \%$ ), Proteobacteria (24.8\%), Bacteroidetes (11.2\%) and Verrucomicrobia (9.4\%). Additionally, Planctomycetes ( $4.4 \%$ ) and Actinobacteria (2.5\%) were found to minor extents.

Most dominant phyla detected in the fecal samples were Bacteroidetes (41.2\%), Firmicutes (34.9\%), Verrucomicrobia (8 \%) and Cyanobacteria (5.4\%). Additionally, Epsilonbacteria (4.3 \%) and Proteobacteria (3.3\%) were found to minor extents.

Of note and in contrast to the fecal samples, the BALF microbiota showed a high percentage of rare and unclassified taxa (Fig. 22).

Taken together, the microbiota composition of lung and gut showed marked differences.

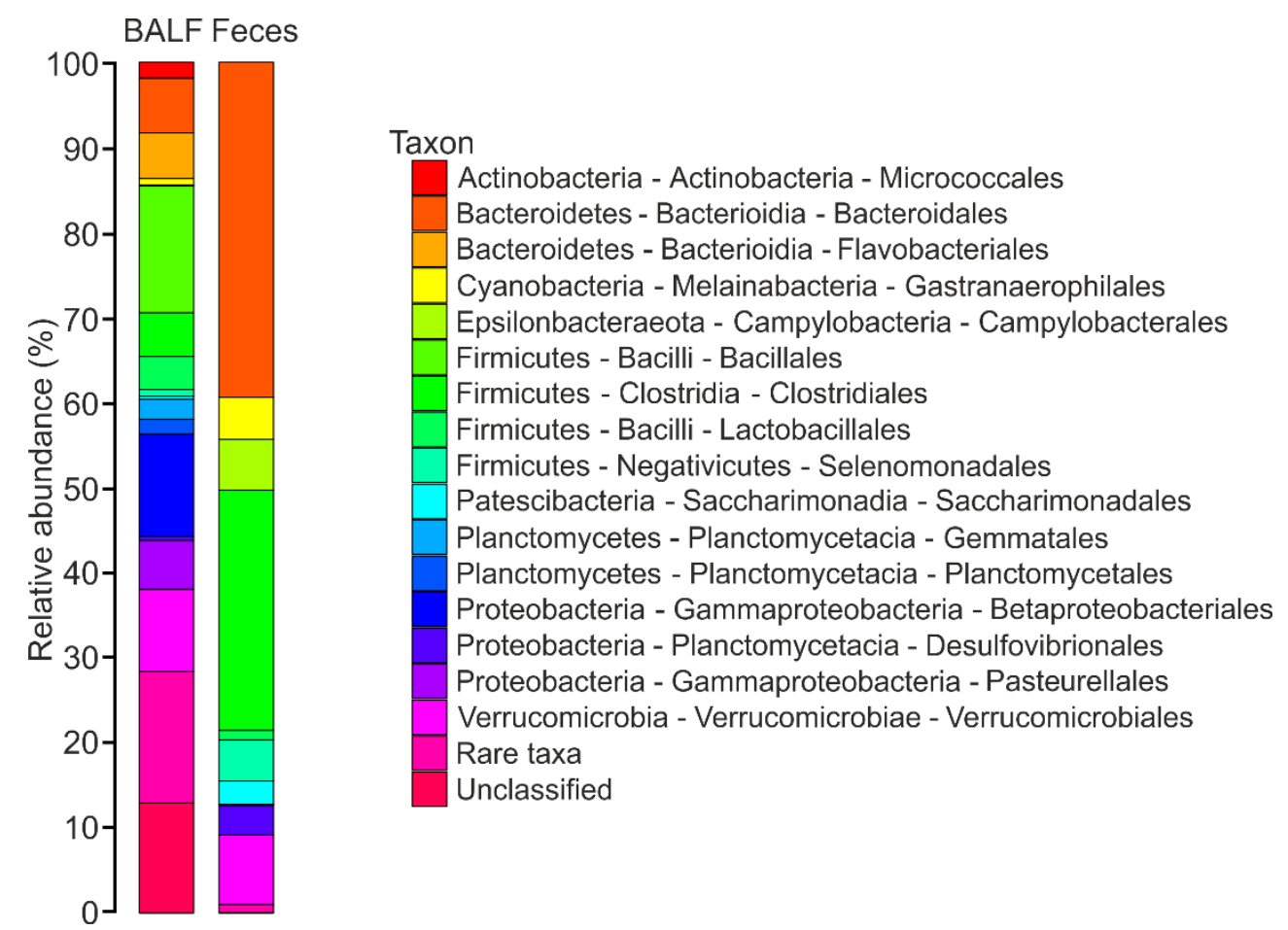

Figure 22: Comparison between lung and gut microbiota composition. BAL fluid (BALF) and feces from rats previously treated i.tr. with PBS for 7 days were collected. DNA from the samples was purified and analyzed via amplicon sequencing of the V3 - V4 165 rRNA region to characterize the bacterial communities based on the operational taxonomic units (OTUs) and exact amplicon sequence variants (ASVs). The relative abundances of bacterial ASVs (phylum - class - order) with $>1.0 \%$ relative abundance per sample are depicted and color-coded. Cumulative data from two independent experiments. BALF: $\mathrm{N}$ = 8; Feces: $\mathrm{N}=6$. Data represented as mean. 


\subsubsection{Neomycin does not interfere with T cell pathogenicity}

Next, we aimed to modify the composition of the lung microbiota by local administration of antibiotics. In order to exclude a systemic effect, the locally acting aminoglycoside neomycin was chosen. Neomycin is a broad-spectrum antibiotic that acts excellently against Gramnegative and partially against Gram-positive bacteria.

First, we tested whether neomycin was toxic for T cells. To this end, $\mathrm{T}_{\text {MBP-EM }}$ cells were activated in vitro in presence of varying concentrations of neomycin (NEO). T cell proliferation was determined on D2, D3 and D4 after antigenic stimulation (Fig. 23B).

We did not observe an impairment in T cell proliferation at any of the tested concentrations ranging from 0 to $10 \mathrm{mg} / \mathrm{mL}$. Only at $100 \mathrm{mg} / \mathrm{mL}$, T cells did not proliferate and the cell numbers progressively decreased (Fig. 23B). Of note, neomycin at this very high dose was barely soluble and the $\mathrm{pH}$ indicator of the medium instantly indicated acidic conditions upon antibiotic addition.

To test whether neomycin may have an effect on T cell pathogenicity in vivo, $\mathrm{T}_{\text {MBP-blast }}$ cells previously stimulated in vitro in presence of neomycin (1 or $10 \mathrm{mg} / \mathrm{mL}$ ) or PBS were adoptively transferred into naïve recipient animals (Fig. 23A). All groups developed a severe EAE starting on D5 p.t. and reaching its peak on D7 p.t. before clinical symptoms started vanishing again until the animals were disease-free on D11 p.t. (Fig. 23C). There was no difference in any of the clinical parameters detectable between any of the tested groups (Fig. $23 D-G)$.

In conclusion, neomycin does not appear to exert toxic effects on TMBP-EM cells. 
A

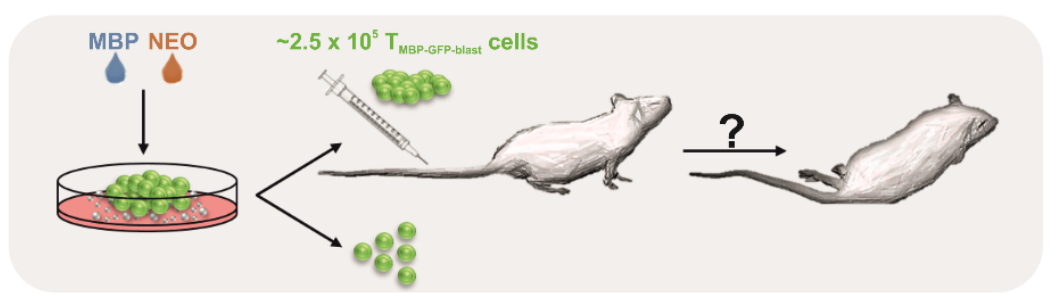

B

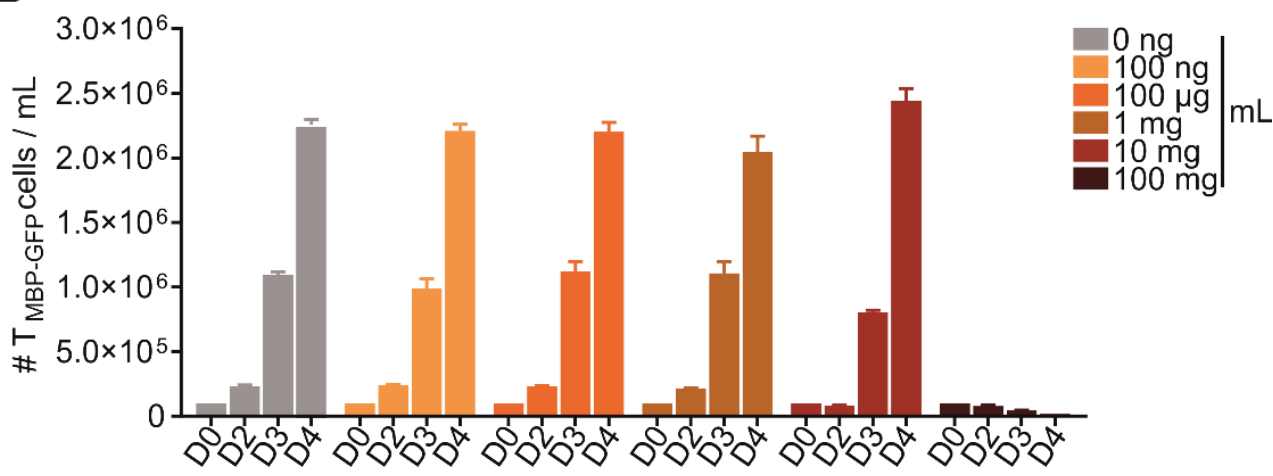

C
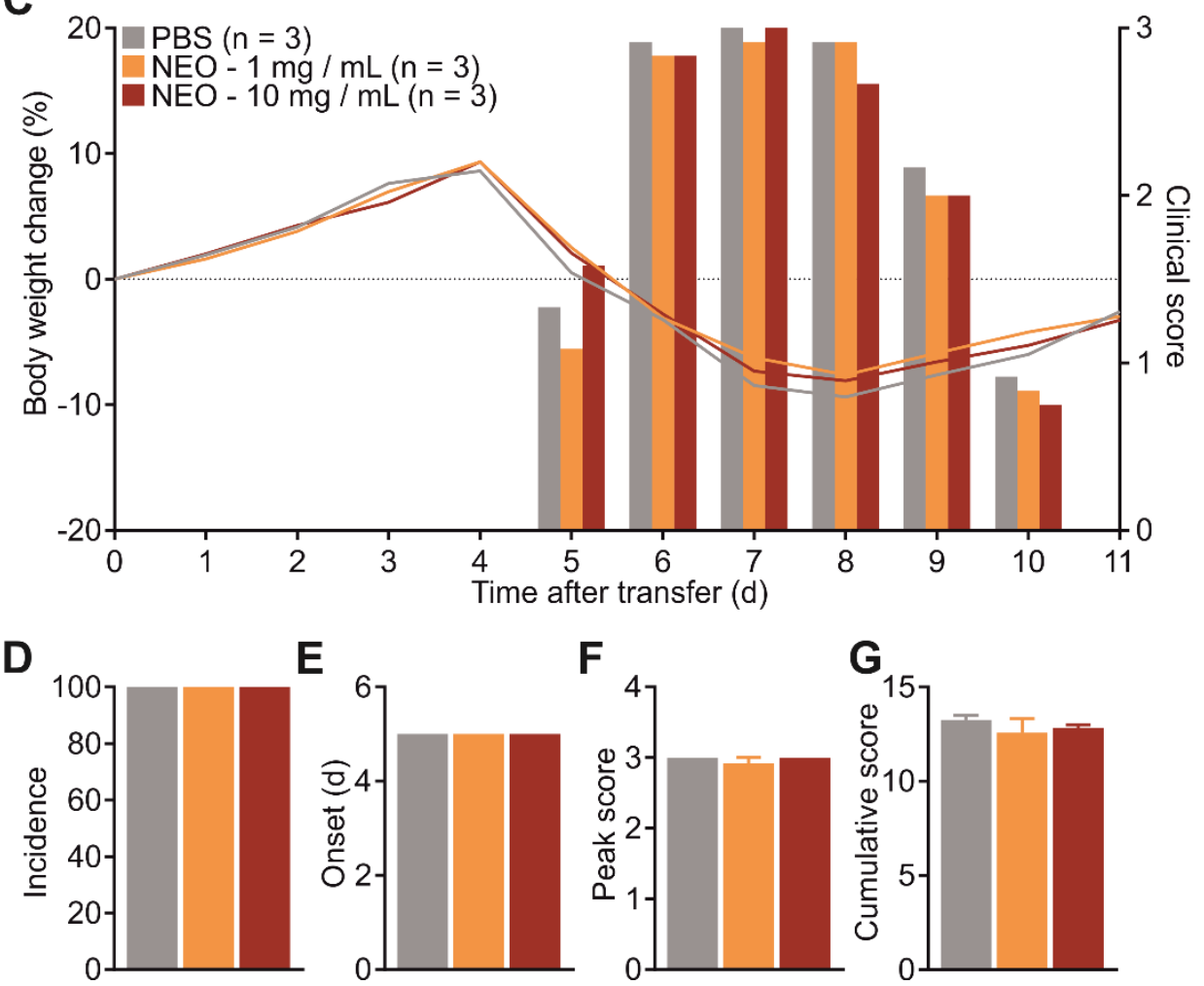

$\mathbf{E}$

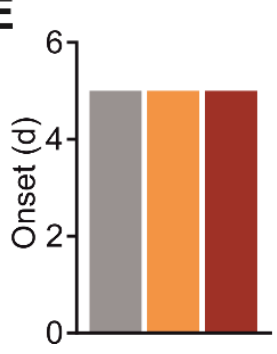

$\mathbf{F}$

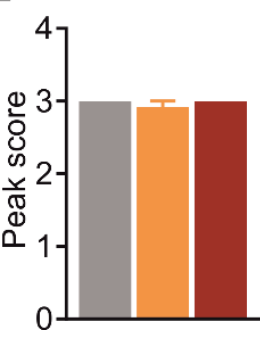

G

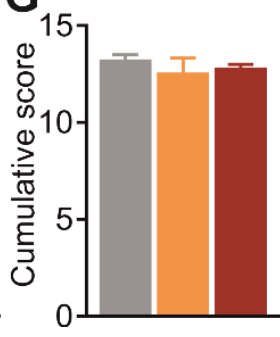

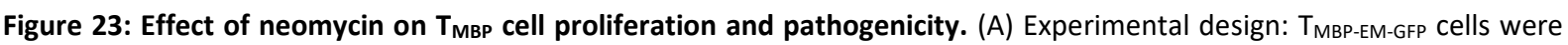
stimulated with irradiated thymocytes and MBP in presence of varying doses of neomycin (NEO). T cell proliferation was determined and $2.5 \times 10^{5} \mathrm{~T}$ cells were adoptively transferred into naïve recipient rats two days post-stimulation ( $\mathrm{T}_{\text {MBP-blast-GFP }}$ cells) to test their pathogenicity following exposure to NEO. (B) Quantification of T cells cultured in presence of NEO ranging from 0 to $100 \mathrm{mg} / \mathrm{mL}$ on D2, 3 and 4 post-stimulation using flow cytometry. (C - G) Clinical outcome of transfer EAE using

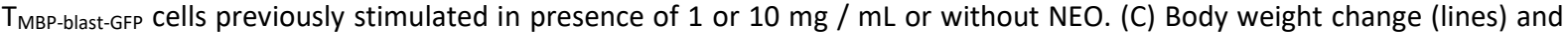
clinical scores (bars) over the course of EAE. (D) Incidence (\%). (E) Average onset (days p.i.). (F) Average peak score. (G) Average cumulative score. Values are represented as mean \pm SEM. Statistical significance was determined via one-way ANOVA with Tukey's multiple comparisons test. 


\subsubsection{Daily administration of neomycin only induces lung inflammation at a dose of $\mathbf{5} \mathbf{~ m g ~ p e r ~}$ day}

Next, we aimed to find a dose of neomycin sufficient to change the composition of the lung microbiota without causing lung tissue irritation. To this end, rats were treated daily with three different concentrations of neomycin $(0.1,1$ or $5 \mathrm{mg}$ ) or PBS for 7 days. On D7 of treatment, the immune cell composition of the lungs was determined.

We observed a significant upregulation of $\mathrm{CD}^{+} \mathrm{T}$ cells (Fig. 24A), $\mathrm{CD} 8^{+} \mathrm{T}$ cells (Fig. 24B) neutrophils (Fig. 24D) and $\mathrm{CD} 11 \mathrm{~b} / \mathrm{c}^{+}$cells (both $\mathrm{MHCII}^{\mathrm{LO}}$ and $\mathrm{MHCII}^{\mathrm{HI}}$; Fig. 24E) in the lungs of the $5 \mathrm{mg}$ treatment group compared to the other groups, indicative of an ongoing inflammation. No difference was observed between the PBS, $0.1 \mathrm{mg}$ and $1 \mathrm{mg}$ neomycin groups in any of the immune cell subsets examined (Fig. 24A-E).

In line with these results, we observed an increase in body weight of about $5-10 \%$ in the PBS, $0.1 \mathrm{mg}$ and $1 \mathrm{mg}$ neomycin group, compared to only $1 \%$ in the $5 \mathrm{mg}$ group (Fig. 24F).

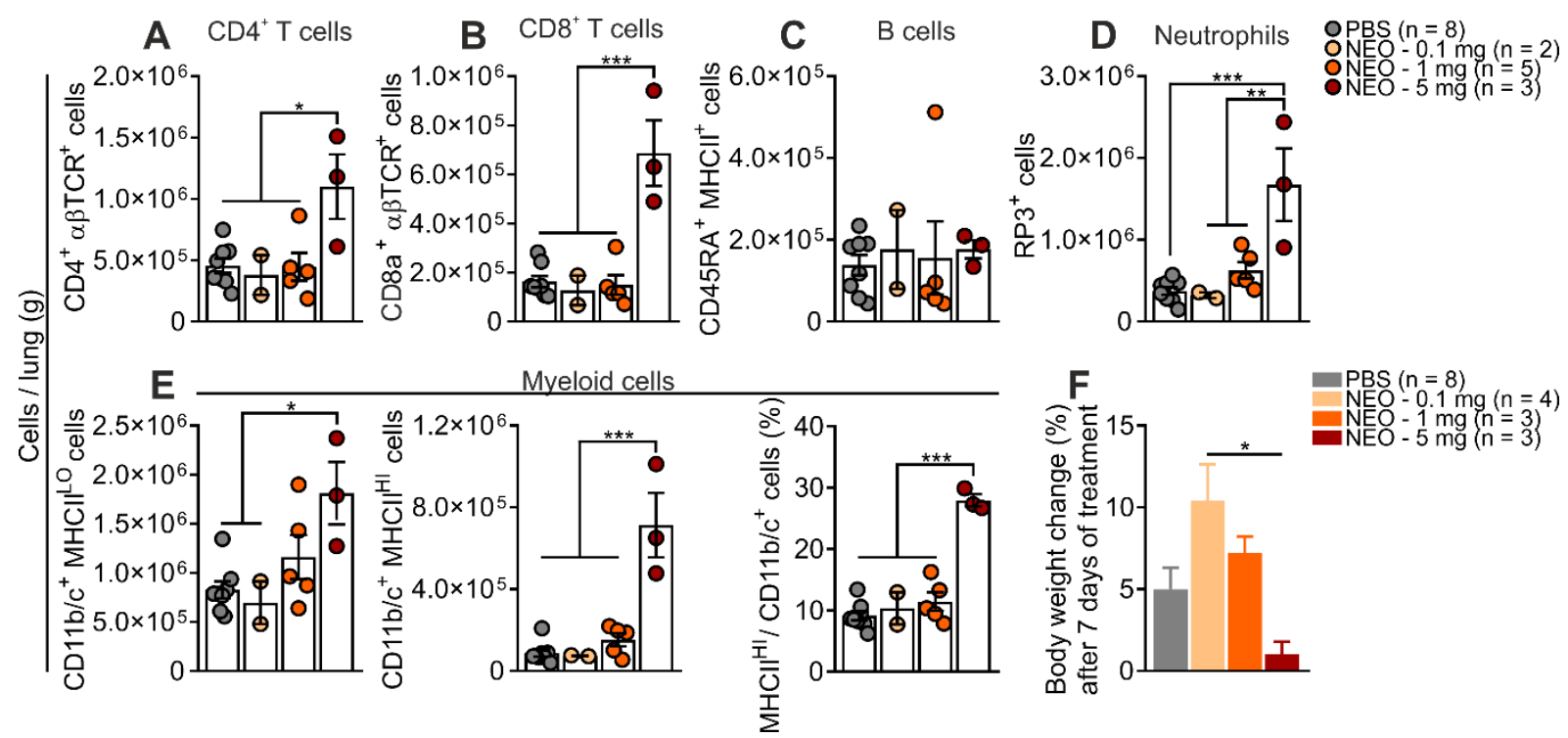

Figure 24: Quantification of immune cells in the lung following neomycin treatment. Rats were i.tr. treated daily with neomycin (NEO; $0.1 \mathrm{mg}, 1 \mathrm{mg}, 5 \mathrm{mg}$ ) or PBS for 7 days. On D7 of treatment, different immune cell populations of the lungs (cells / g) were quantified as a measure of inflammation using antibody labeling and flow cytometry. The following antibody combinations were used to label the corresponding immune cell types: (A) CD4 ${ }^{+} T$ cells: $C D 4^{+} \alpha \beta T C R^{+}$. (B) CD8 ${ }^{+}$T cells: CD8a ${ }^{+}$

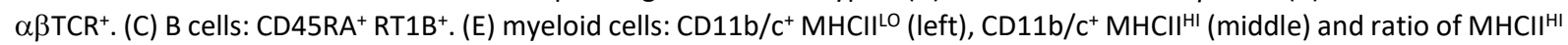
$/ \mathrm{CD} 11 \mathrm{~b} / \mathrm{c}^{+}$cells (right). (F) Body weight change of the four treatment groups after 7 days of treatment (\%). Cumulative data from three independent experiments. Values are represented as mean \pm SEM. Statistical significance was determined via one-way ANOVA with Tukey's multiple comparisons test. ${ }^{*} p<0.05 ;{ }^{* *} p<0.01 ; * * p<0.001$.

\subsubsection{Neomycin treatment appears to alter the lung microbiota composition in a dose- dependent manner}

Next, we investigated if neomycin can modify the composition of the lung microbiota. To this end, rats were i.tr. treated daily with $0.1,1$ or 5 mg neomycin or PBS (see 3.2.1) for 7 days. On D7 of treatment, BAL fluid (BALF) and feces were collected and analyzed via NGS as previously described (3.2.1). 
With increasing neomycin concentration, the composition of the lung microbiota appeared to change: the percentage of some of the Bacteroidetes classes of Bacteroidia for example appeared to increase whereas some Firmicutes classes as well as some Proteobacteria classes of Gammaproteobacteria appeared to decrease (Fig. 25).

In contrast, i.tr. neomycin treatment did not appear to affect the overall composition of the gut microbiota (Fig. 25).
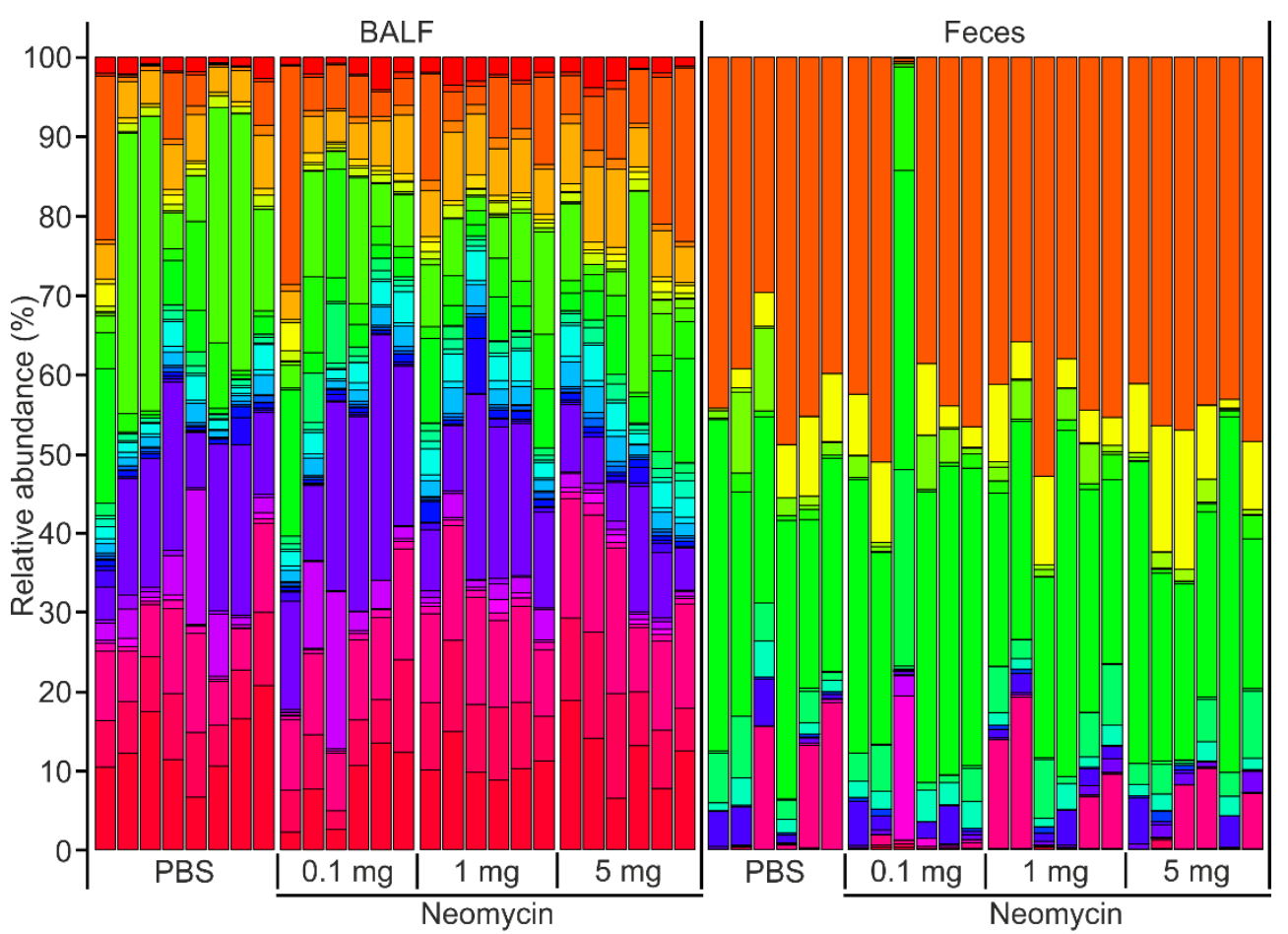

Taxon
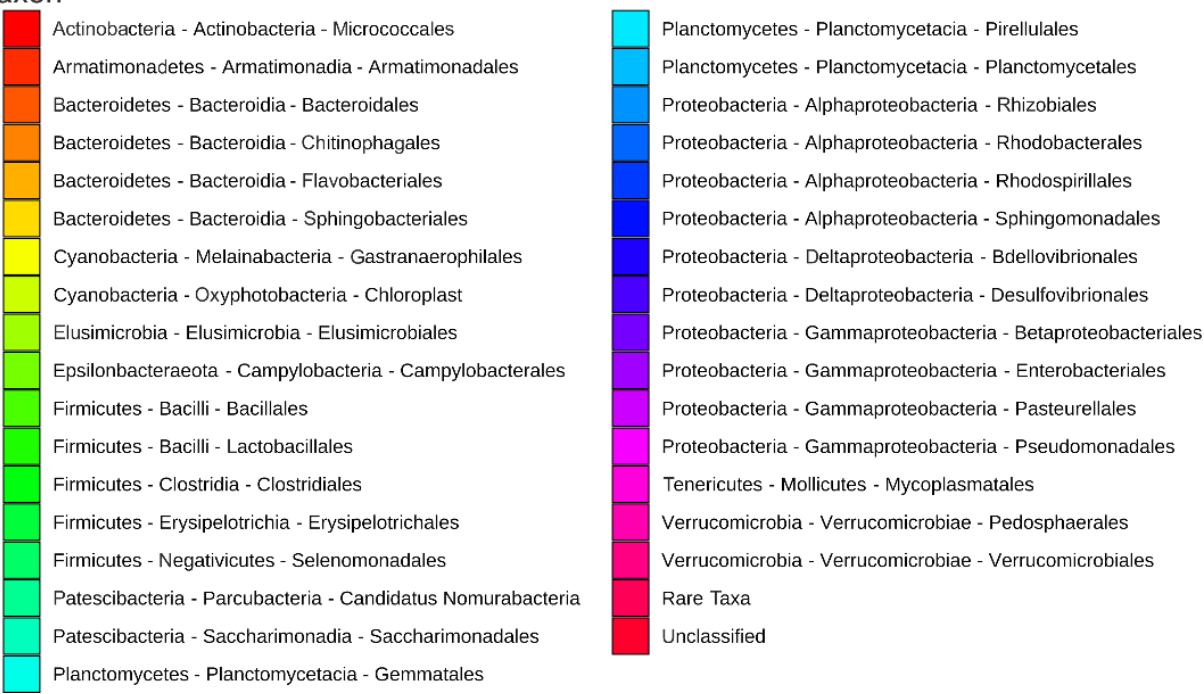

Figure 25: Effect of i.tr. neomycin treatment on lung and gut microbiota composition. BAL fluid (BALF) and feces from rats previously treated i.tr. with neomycin (NEO; $0.1 \mathrm{mg}, 1 \mathrm{mg}, 5 \mathrm{mg}$ ) or PBS for 7 days were collected. Sample preparation, analysis and depiction as in Fig. 22 with the exception that each column represents the microbial composition of one individual. Combined data from two independent experiments. 
We further analyzed the neomycin-induced change of the microbial composition in the lung in more detail by focusing on the 15 most abundant taxa on genus level.

Bacillus (phylum Firmicutes) exhibited a decrease in abundance at all tested concentrations. Achromobacter, Rodentibacter and Streptococcus were reduced upon treatment with 1 and 5 mg of neomycin in a dose dependent manner. The genera Helicobacter, Prosthecobacter and Flavobacterium, in contrast, were increased in a dose-dependent manner (Fig. 26A).

In the gut, only moderate changes were detected: Alistepes and Helicobacter represented the most up- and downregulated bacteria, respectively. The changes in abundance were dosedependent (Fig. 26A).

In order to quantify the effect of the antibiotic treatment, we next determined the microbial diversity ( $\alpha$-diversity) of BALF and feces using four indices: Shannon, Simpson, Invsimpson and Chao1. In all cases, a general trend toward an increased $\alpha$-diversity of the BALF microbiota induced by i.tr. neomycin treatment could be observed. This increase was in general dosedependent and significant in the $1 \mathrm{mg}$ dose for the Simpson index and in the $5 \mathrm{mg}$ dose for the Chao1 index.

No tendency toward an altered $\alpha$-diversity could be observed for the fecal samples. Instead, $\alpha$-diversity of the three neomycin groups was very similar to the PBS group (Fig. 26B - E). Taken together, neomycin treatment altered the composition of the BALF but not fecal microbiota in a dose-dependent manner and tended to induce an increase in $\alpha$-diversity. 
A

Firmicutes; Bacillus

Proteobacteria; Achromobacter

Firmicutes, Lachnospiraceae NK4A136 group

Bacteroidetes; Prevotellaceae UCG-001

Bacteroidetes; Prevotellaceae NK3B31 group

Bacteroidetes; Alistipes

Bacteroidetes; Flavobacterium

Verrucomicrobia; Akkermansia

Firmicutes; Phascolarctobacterium

Proteobacteria; Rodentibacter

Verrucomicrobia; Prosthecobacter

Firmicutes; Streptococcus

Epsilonbacteraeota; Helicobacter

Firmicutes; Lactobacillus

Firmicutes; Oscillibacter

BALF

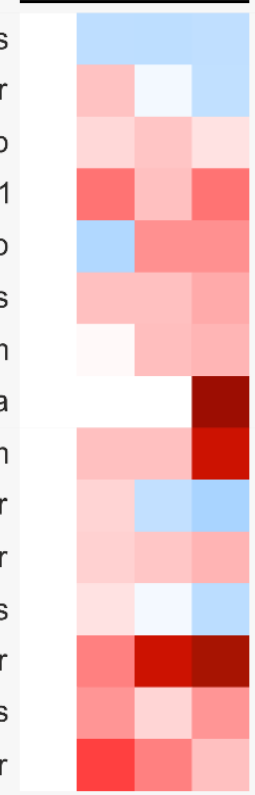

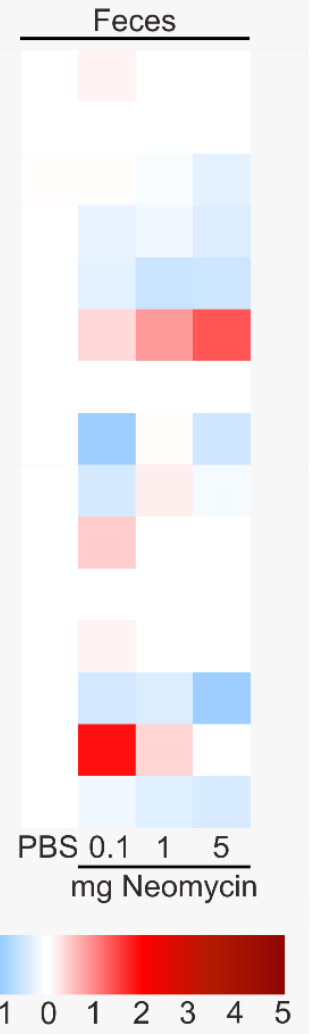

$\begin{array}{llllllll}-2 & -1 & 0 & 1 & 2 & 3 & 4 & 5\end{array}$
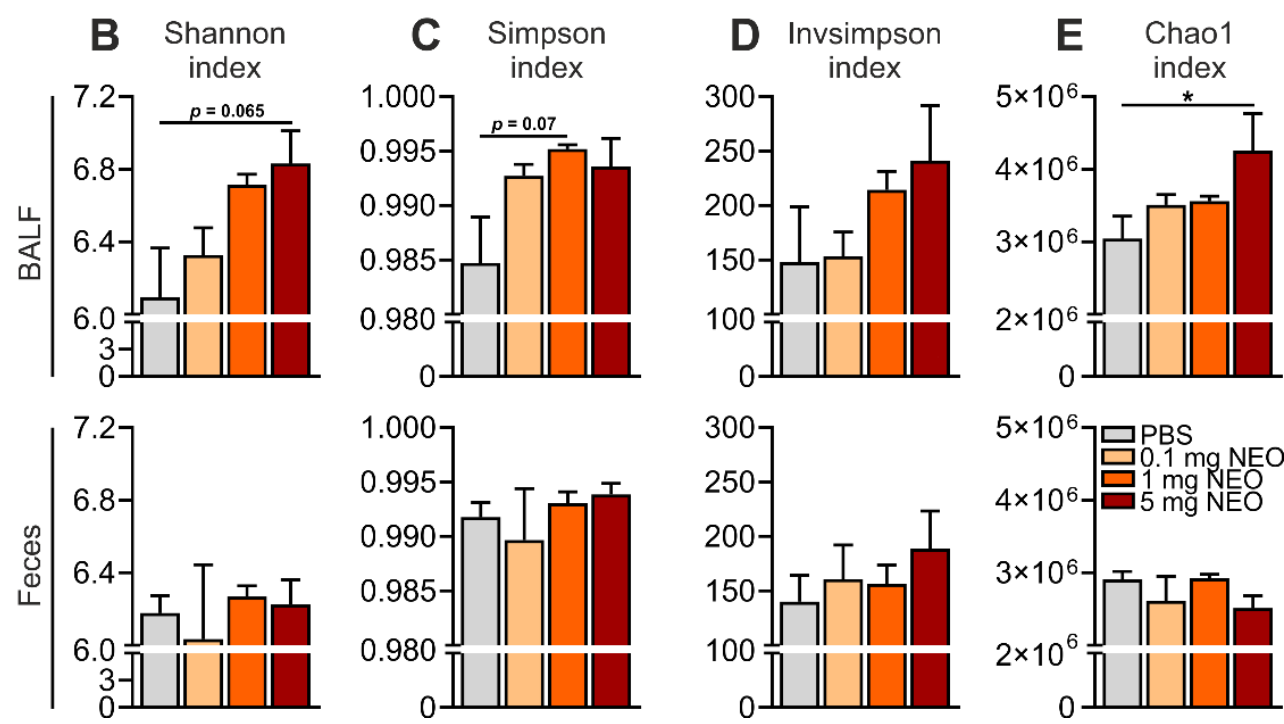

Figure 26: Effect of i.tr. neomycin treatment on lung and gut microbiota genera and $\boldsymbol{\alpha}$-diversity. Sample collection, preparation and analysis as described in Fig. 22. (A) The heat map represents the average relative fold changes in \% read abundance of the 15 most abundant genera of BAL fluid (BALF) and feces normalized to the corresponding PBS groups. (B D) Indices (as indicated) for $\alpha$-diversity of the microbiota composition of BALF (upper row) and feces (lower row). Cumulative data from two independent experiments. Values are represented as mean \pm SEM. Statistical significance was determined via one-way ANOVA with Tukey's multiple comparisons test. * $p<0.05$. 


\subsubsection{Neomycin treatment prevents i.tr. induced EAE}

Having observed that intrapulmonary neomycin treatment changes the composition of the local microbiota, we wanted to test its potential effect on i.tr. induced EAE. To this end, rats were i.tr. treated daily with neomycin $(0.1,1$ or $5 \mathrm{mg}$ ) or PBS for 7 days. Non-treated animals (CTRL) were used as additional control. On D7 of treatment, the animals were immunized with the LO-OPT MBP dose following TMBP-EM cell transfer. On D7 p.i., the peak of disease, the distribution of the transferred T cells in different organs was determined (Fig. 27A).

Strikingly, while the animals of the two control groups and the $0.1 \mathrm{mg}$ neomycin group developed typical EAE with very similar clinical parameters (incidence: $100 \%$; average onset: D5.7 - D6.5 p.i.; average peak score: 2.1 -2.2), daily treatment with $1 \mathrm{mg}$ and $5 \mathrm{mg}$ neomycin completely prevented EAE (Fig. 27B - E).

In line with the clinical data, the number of transferred T cells detected in the spinal cord (CNS) of the control groups and $0.1 \mathrm{mg}$ neomycin group on D7 p.i. were similar and ranged from $2.62 \times 10^{5} \pm 5.67 \times 10^{5} / \mathrm{g}$ in the CTRL to $3.16 \times 10^{5} \pm 5.67 \times 10^{5} / \mathrm{g}$ in the PBS group. T cell numbers in the CNS of the $1 \mathrm{mg}$ and $5 \mathrm{mg}$ neomycin groups in contrast were $4.7 \times 10^{4} \pm 2.0 \mathrm{x}$ $10^{4} / \mathrm{g}$ and $3.1 \times 10^{4} \pm 1.7 \times 10^{4} / \mathrm{g}$, respectively, and thus significantly lower compared to the other three groups (Fig. 27K). There was no significant difference in T cell numbers between any of the groups in lung, mediastinal lymph nodes, inguinal lymph nodes, spleen and blood (Fig. 27F-J).

These results clearly demonstrate that intrapulmonary neomycin treatment can efficiently block EAE, starting at a dose of $1 \mathrm{mg}$ per day. 

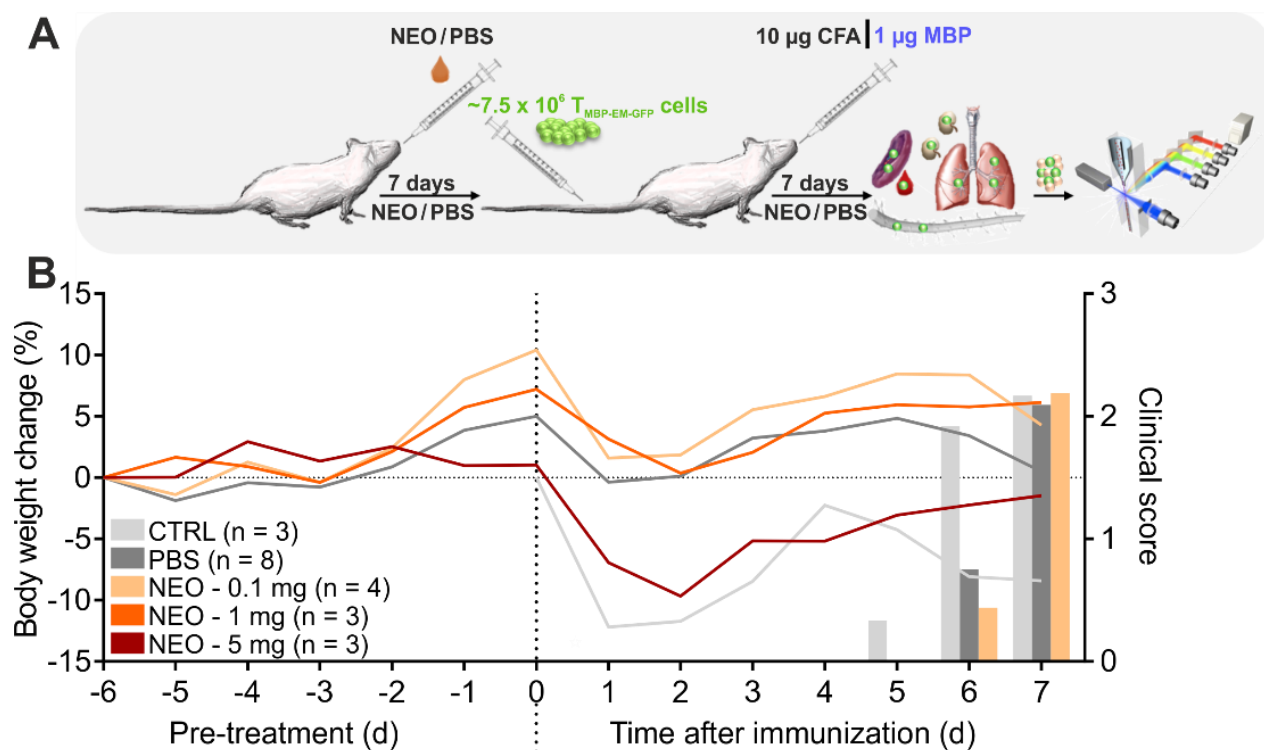

C

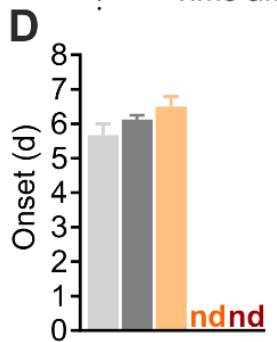

E
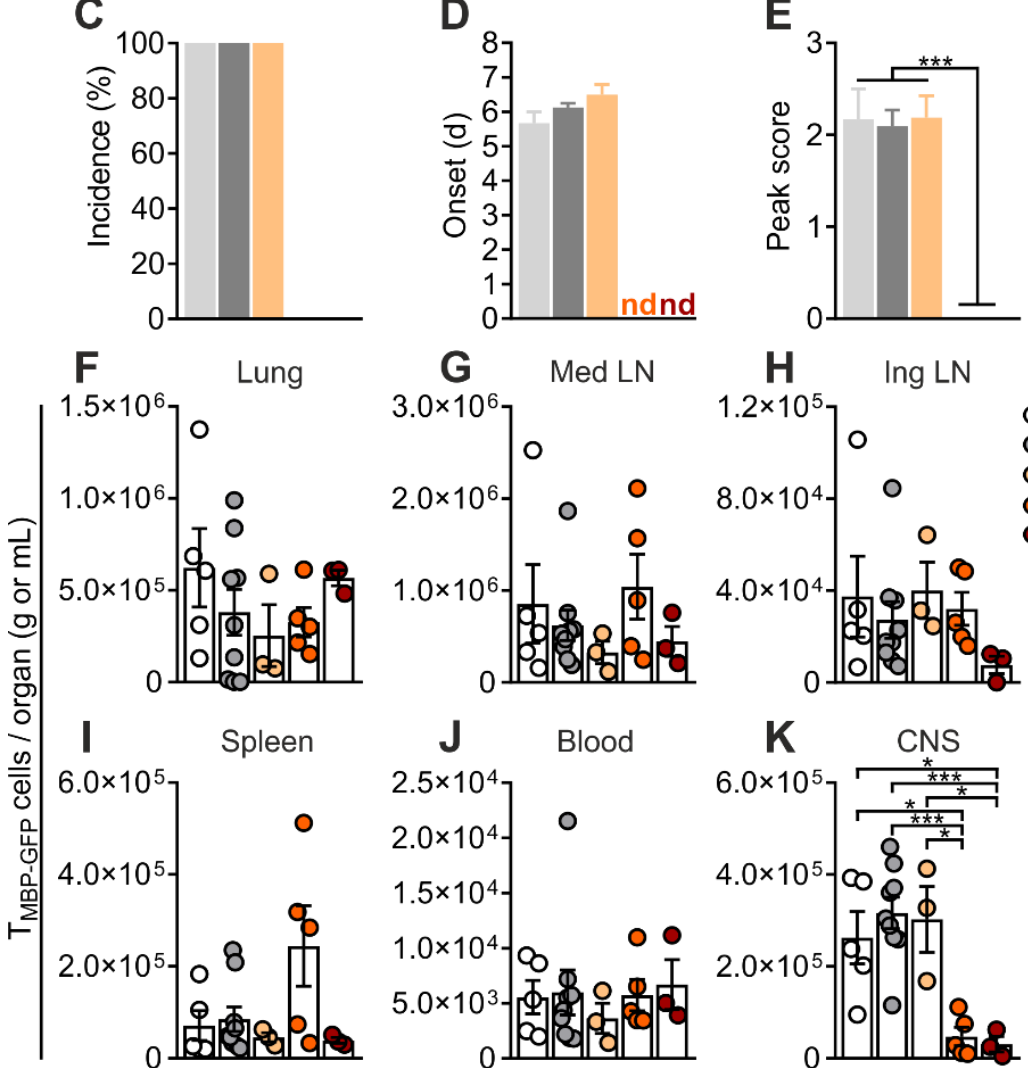

H Ing LN

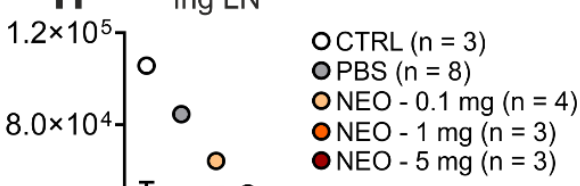

$4.0 \times 1$
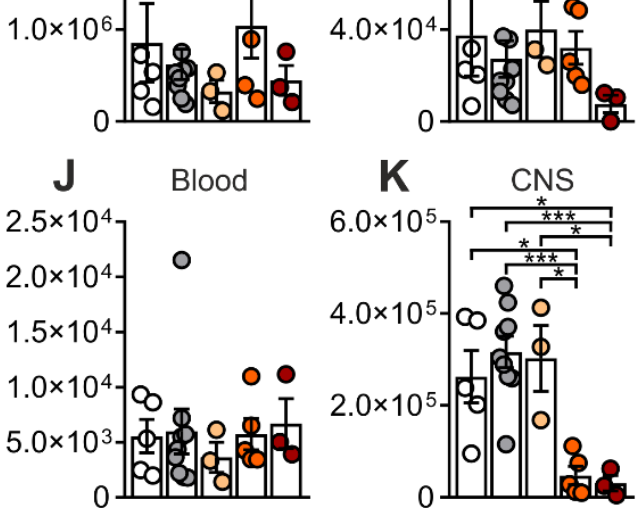

Figure 27: Characterization of the effect of i.tr. neomycin treatment on i.tr. induced EAE. (A) Experimental design: Rats were i.tr. treated daily with neomycin (NEO; $0.1 \mathrm{mg}, 1 \mathrm{mg}, 5 \mathrm{mg}$ ) or PBS (PBS) for 7 days. One group of animals remained untreated

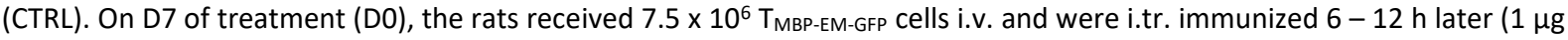
MBP and $10 \mu \mathrm{g}$ CFA / $300 \mathrm{~g}$ body weight). The rats were continued to be treated daily and the clinical course of EAE was monitored for 7 days. On D7 p.i., the distribution of the transferred T cells in different organs was determined using flow cytometry. (B) Body weight change (lines) and clinical scores (bars) over the course of EAE. (C) Incidence (\%). (D) Average onset (days p.i.). (E) Average peak score. $(F-K) T$ cells per $g$ or $\mathrm{mL}$ detected in the indicated organs on D7 p.i. Cumulative data from two $(B-E)$ or three $(F-K)$ independent experiments. $n d=$ not determined. Values are represented as mean $\pm S E M$. Statistical significance was determined via one-way ANOVA with Tukey's multiple comparisons test. * $p<0.05 ;{ }^{* * *} p<0.001$. 


\subsubsection{Neomycin treatment per os does not appear to affect i.tr. induced EAE}

In order to confirm that the observed preventive effect of neomycin on EAE upon i.tr. treatment is lung-specific and not gut-dependent, rats were treated with $1 \mathrm{mg}$ neomycin or PBS via gavage (per os; p.o.) for 7 days. Because the surface of the gut mucosa is by far larger than the lung mucosa, we also included a 10x (10 mg) and 100x (100 mg) higher neomycin dose. The rats were i.tr. immunized with the LO-OPT MBP dose following TMBP-EM $_{\text {cell }}$ transfer. At the peak of $E A E$, the distribution of the transferred $T$ cells in different organs was determined.

Treatment with $100 \mathrm{mg}$ of neomycin resulted in a decreased survival rate of about $27.6 \%$ by D7 of treatment, indicating a toxic effect. The survival rate of the other groups was $100 \%$ (Fig. 28A). T cell numbers in all the peripheral organs were comparable between all groups and no significant differences could be detected (Fig. 28B - F). There was a slight tendency toward a reduced number of $\mathrm{T}$ cells in the $100 \mathrm{mg}$ neomycin group in mediastinal lymph nodes, spleen and blood (Fig. 28C, E, F). In the spinal cord (CNS), a significant reduction of T cells was detected only at $100 \mathrm{mg}$ neomycin. No differences could be observed between the PBS, $1 \mathrm{mg}$ and $10 \mathrm{mg}$ neomycin groups (Fig. 28G).

The generally low T cell numbers in different organs of the $100 \mathrm{mg}$ neomycin group as well as the decreased survival rate might have resulted from a potential toxic effect of neomycin treatment at very high doses.

In conclusion, the i.tr. dose of neomycin sufficient to prevent T cell infiltration into the CNS did not evoke any effect when administered p.o., indicating that a lung-associated mechanism could be responsible for the blockage of i.tr. induced EAE. 

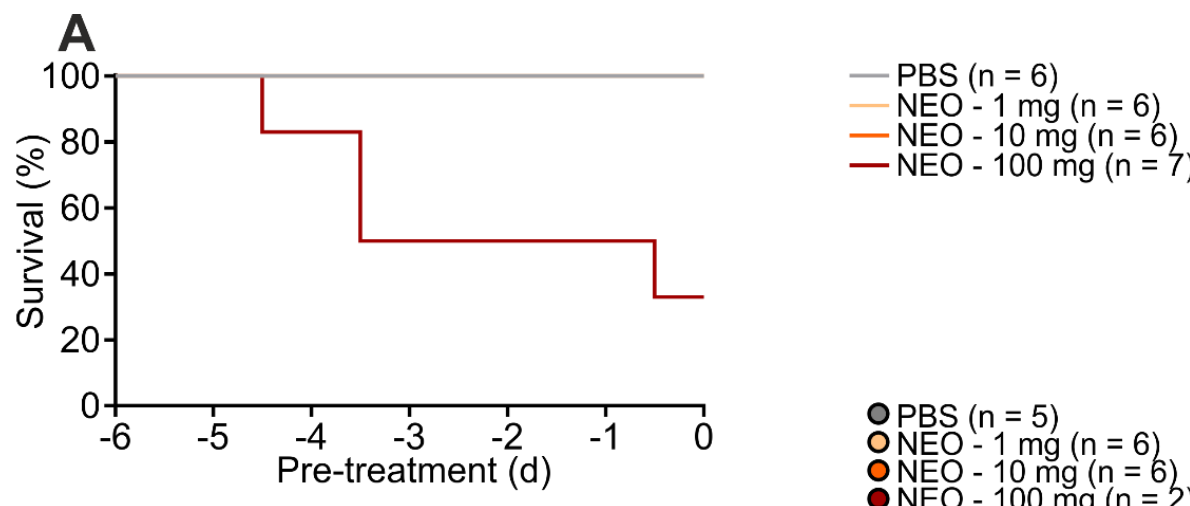

- NEO - $100 \mathrm{mg}(\mathrm{n}=7)$
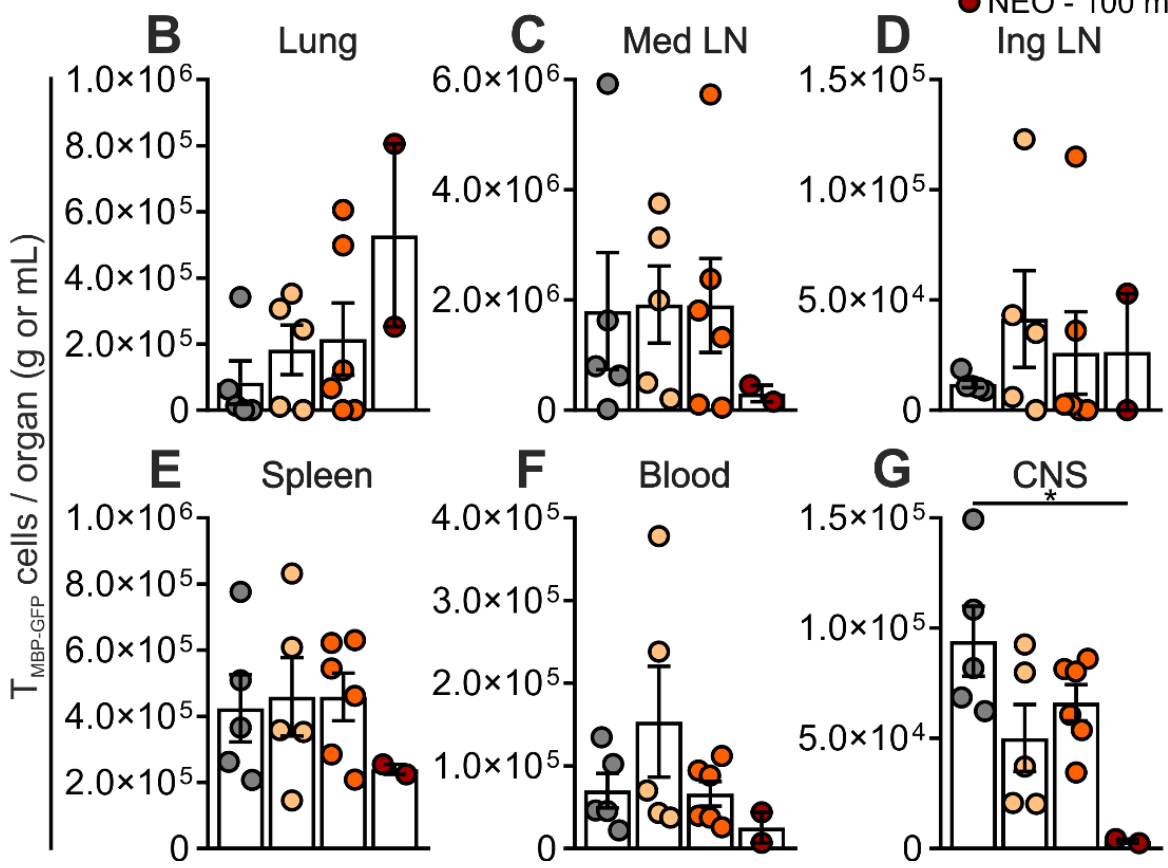

OPBS $(n=5)$

ONEO - $1 \mathrm{mg}(\mathrm{n}=6)$

O NEO - $10 \mathrm{mg}(\mathrm{n}=6)$ Ing LN

Figure 28: Characterization of the effect of p.o. neomycin treatment on i.tr. induced EAE. Rats were treated p.o. daily with neomycin (NEO; $1 \mathrm{mg} ; 10 \mathrm{mg} ; 100 \mathrm{mg}$ ) or PBS for 7 days. On D7 of treatment (D0), the rats received $7.5 \times 10^{6} \mathrm{~T}_{\mathrm{MBP} \text {-EM-GFP Cells }}$ i.v. and were i.tr. immunized $6-12 \mathrm{~h}$ later ( $1 \mu \mathrm{g} \mathrm{MBP}$ and $10 \mu \mathrm{g}$ CFA / $300 \mathrm{~g}$ body weight). The rats were continued to be treated daily. On D7 p.i., the distribution of transferred T cells in different organs was determined using flow cytometry. (A) Survival (\%) over the course of 7 days of NEO treatment (B $-\mathrm{G})$. Transferred T cells per $\mathrm{g}$ or $\mathrm{mL}$ detected in the indicated organs on D7 p.i. Cumulative data from two independent experiments. Values are represented as mean \pm SEM. Statistical significance was determined via one-way ANOVA with Tukey's multiple comparisons test. ${ }^{*} p<0.05$.

\subsubsection{Activation of $T_{M B P-E M}$ cells in the lungs of neomycin-treated rats is not impaired}

Next, we investigated whether i.tr. neomycin treatment impairs TMBP-EM cell activation within the lung. To this end, rats treated with neomycin or PBS for 7 days were i.tr. immunized with the LO-OPT dose of MBP following TMBP-EM cell transfer. Non-immunized animals (NAI) and animals immunized with CFA alone (CFA) served as controls. $24 \mathrm{~h}$ later, the activation state of T cells sorted from the lung was determined.

Independent of the treatment, the expression of both IFN $\gamma$ and IL-17 was highly upregulated following i.tr. immunization with the LO-OPT MBP dose. We did not observe any difference between neomycin and PBS treatment in any of the immunization or control conditions (Fig 29A, B). 
In order to further corroborate these data, we performed transcriptome analysis of the transferred T cells sorted from PBS or neomycin-treated animals before and 24 hours after i.tr. immunization with the LO-OPT dose of MBP. Principle cluster analysis (PCA) revealed a time point-dependent but treatment-independent clustering of $T$ cells sorted from both groups on D0 and D1 p.i. with the exception of one outlier in the neomycin group on D0 (Fig. 29C). Moreover, normalized counts of IFN $\gamma$ and IL-17 expression confirmed an upregulation of the expression of both genes on D1 p.i. that was independent of neomycin treatment (Fig. 29D, E). We also compared the global expression profile of T cells sorted $24 \mathrm{~h}$ p.i. and observed that indeed not a single gene was regulated differently between the PBS- and the neomycintreated group.

In summary, these data indicate that the process of intrapulmonary $T_{M B P-E M}$ cell activation was not affected by neomycin treatment.
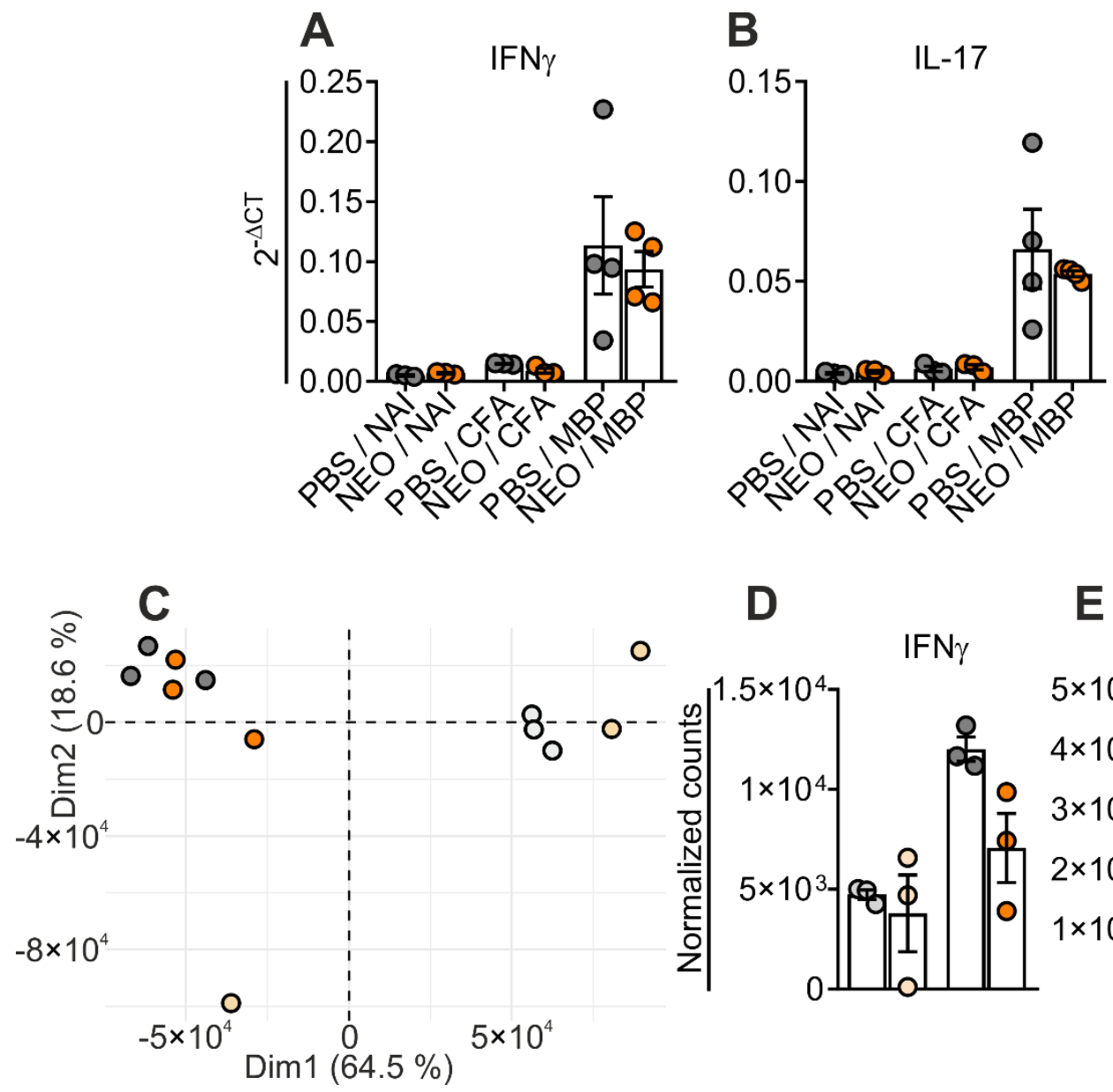

D E

IFN $\gamma$

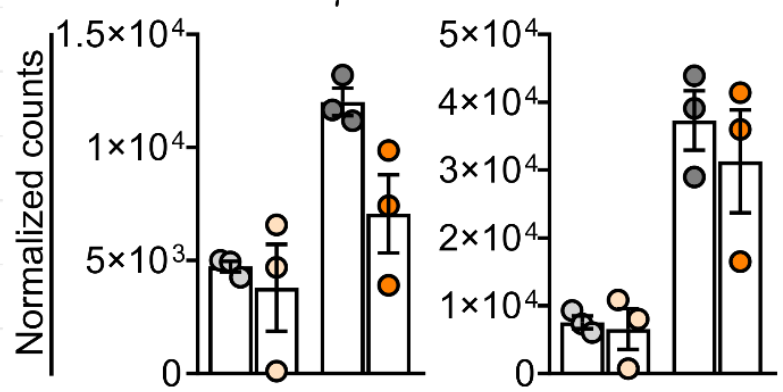

Figure 29: Effect of i.tr. neomycin treatment on $\mathrm{T}_{\text {MBP-EM }}$ cell activation in the lung. Rats were i.tr. treated daily with neomycin

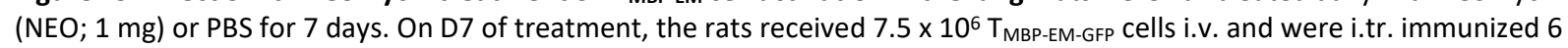
- $12 \mathrm{~h}$ later with (MBP; $1 \mu \mathrm{g}$ MBP and $10 \mu \mathrm{g}$ CFA / $300 \mathrm{~g}$ body weight; A - E) or without (CFA; $0 \mu \mathrm{g}$ MBP and $10 \mu \mathrm{g}$ CFA / 300 $\mathrm{g}$ body weight; LO-OPT; $A-B$ ) MBP. One group of each treatment was left non-immunized (NAI; $A-E)$. (A-B) $24 \mathrm{~h}$ later, transferred $T$ cells were sorted from the lungs. T cell activation on mRNA level was determined using qPCR based on the expression of IFN $\gamma(A)$ and IL-17 (B). Expression of all genes $\left(2^{-\Delta C T}\right)$ was normalized to $\beta$-actin expression of the same samples. (C - E) $12 \mathrm{~h}$ p.t. (D0; non-immunized) or $24 \mathrm{~h}$ p.i. (D1; MBP-immunized), transferred T cells were sorted from the lungs of the NEO and PBS group and underwent transcriptome analysis using NGS. (C) Principle cluster analysis (PCA) of the expression profiles of the sorted T cells. (D - E) Corresponding expression (normalized counts) of IFN $\gamma$ (D) and IL-17 (E). Values are represented as mean \pm SEM. Statistical significance was determined via unpaired two-tailed $t$-test. 


\subsubsection{Intrapulmonary treatment with neomycin blocks EAE independent of the site of immunization}

Next, we aimed to investigate whether the preventive effect of i.tr. neomycin treatment on $E A E$ is dependent on the site of treatment and / or on the site of immunization. To this end, rats were treated with neomycin or PBS administered i.tr. or s.c. for 7 days and subsequently i.tr. or s.c. immunized following TMBP-EM cell transfer. In addition to the clinical outcome, distribution of the transferred $T$ cells in different organs around EAE onset (D8 p.i.) was determined.

In the animals s.c. treated with neomycin or PBS and i.tr. immunized, no difference in the clinical course could be observed between the groups (Fig. 30A, B). All animals developed EAE with an average onset around D7 p.i., average peak scores of $1.7 \pm 0.09$ in the PBS and $1.69 \pm$ 0.12 in the neomycin group and comparable respective cumulative scores of $3.9 \pm 0.32$ and $3.94 \pm 0.53$ (Fig. 30A, B). Accordingly, no differences in transferred T cell numbers could be observed in the spinal cord or any other of the organs examined (Fig. 31A).

Similarly, the clinical EAE course was almost identical in s.c. immunized animals s.c. treated with neomycin or PBS (Fig. 30C, D). All animals developed EAE starting around D9 p.i. The average peak score of the PBS group and the neomycin group was about $2.58 \pm 0.3$ and $2.5 \pm$ 0.38 , respectively (Fig. 30C, D). The respective average cumulative scores were $10.08 \pm 1.37$ and $9.92 \pm 1.91$. In line with the clinical outcome, no significant differences between $\mathrm{T}$ cell numbers in any of the organs examined could be observed (Fig. 31B).

In contrast, a strong amelioration of EAE was observed in the animals i.tr. treated with neomycin and s.c. immunized (Fig. 30E, F). The incidence of the neomycin-treated group was reduced to $75 \%$ compared to $100 \%$ in the PBS group. EAE onset was significantly delayed to D11 p.i. compared to D9 p.i. The peak score of the neomycin group was about $0.5 \pm 0.2$ compared to about $2.81 \pm 0.12$ in the PBS group and the cumulative score about $1.13 \pm 0.43$ compared to about $11.69 \pm 1$ (Fig. 30E, F). In line with the clinical data, we observed a significantly lower number of T cells in the spinal cords of the neomycin group whereas there was no difference in any of the peripheral organs (Fig. 31C).

In conclusion, the results indicate that intrapulmonary administration of neomycin is crucial for the prevention or amelioration of EAE. This effect appears to be at least partially independent of the site of EAE induction. 
A s.c. Treatment / i.tr. Immunization

\section{B}
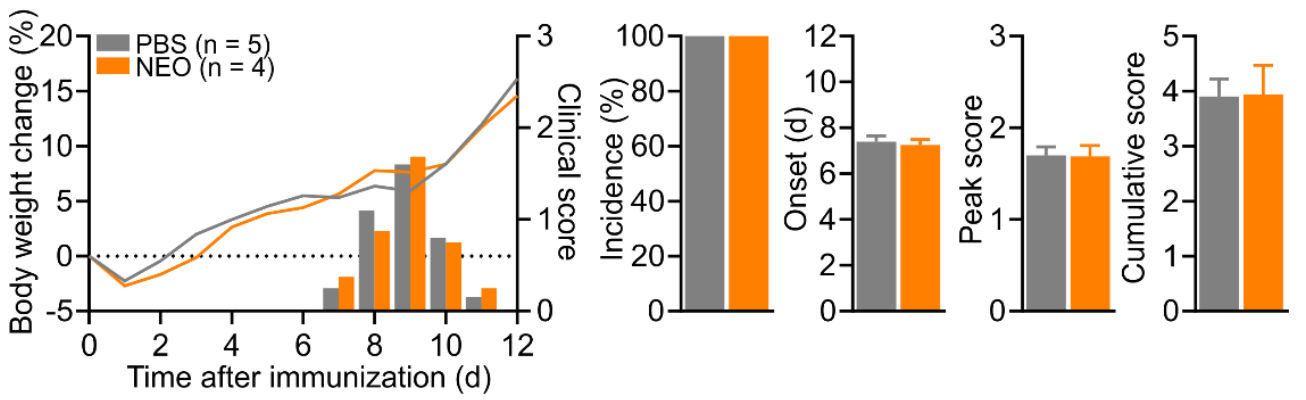

C s.c. Treatment / s.c. Immunization

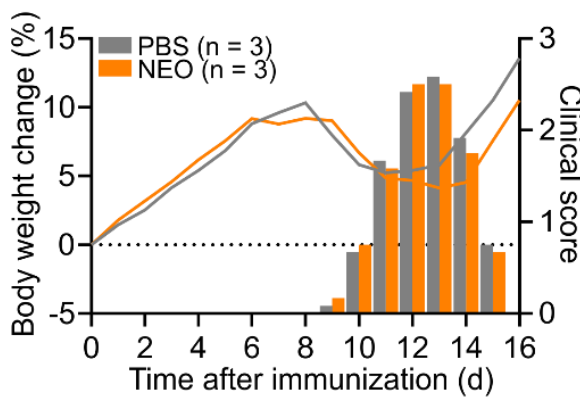

D
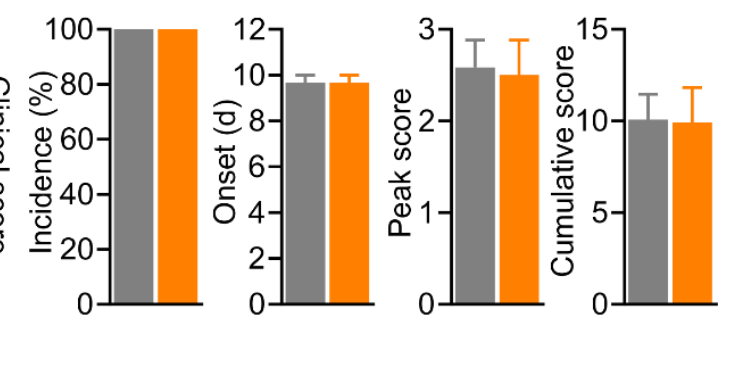

E i.tr. Treatment / s.c. Immunization

\section{$\mathbf{F}$}
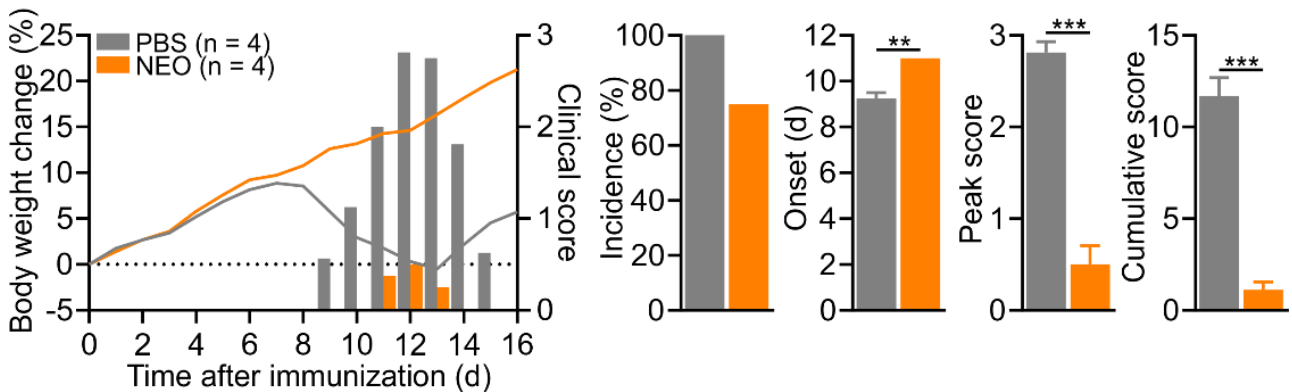

Figure 30: Examination of the relevance of treatment and immunization site for the neomycin effect on EAE. Rats were treated i.tr. or s.c. daily with neomycin (NEO; $1 \mathrm{mg}$ ) or PBS for 7 days. On D7 of treatment, the rats received $7.5 \times 10^{6} \mathrm{~T}_{\mathrm{MBP}-\mathrm{EM} \text { - }}$ GFP cells i.v. and were i.tr. ( $1 \mu \mathrm{g} \mathrm{MBP}$ and $10 \mu \mathrm{g}$ CFA / $300 \mathrm{~g}$ body weight) or s.c. (50 $\mu \mathrm{g}$ MBP and $50 \mu \mathrm{g}$ CFA / $300 \mathrm{~g}$ body weight) immunized 6-12 $\mathrm{h}$ later. The rats were continued to be treated daily via the same route and the clinical course of EAE was monitored until the animals had fully recovered. The following combinations of treatment site and immunization site were examined: $(A-B)$ S.c. treatment and i.tr. immunization. (C - D) S.c. treatment and s.c. immunization. ( $E-F)$ l.tr. treatment and s.c. immunization. Graphical depiction of EAE course and clinical parameters arranged from left to right: (A, C, E) body weight change (lines) and clinical scores (bars) over the course of EAE; (B, D, F) Incidence (\%); Average onset (days p.i.); Average peak score. Cumulative data from two independent experiments. Values are represented as mean \pm SEM. Statistical significance was determined via unpaired two-tailed $t$-test. $* * p<0.01 ; * * p<0.001$. 
A s.c. Treatment / i.tr. Immunization

OPBS $(n=3)$

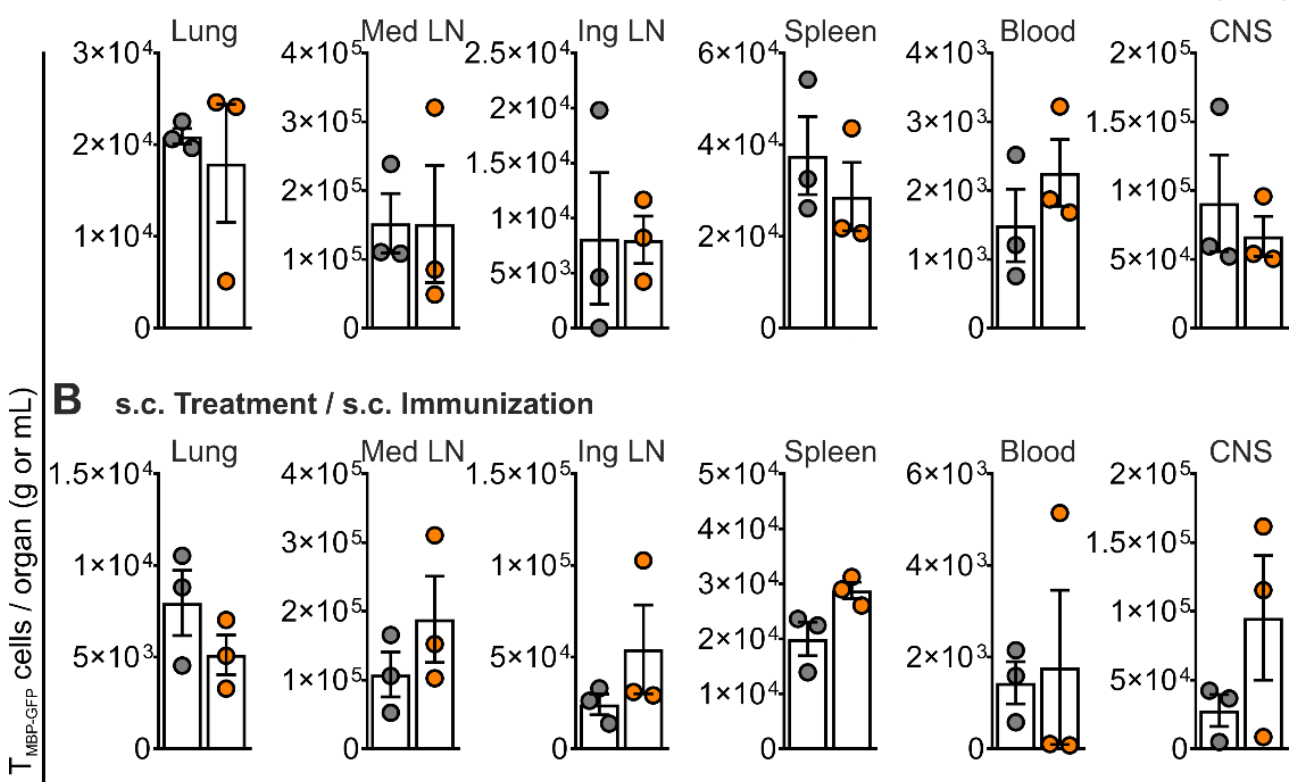

C i.tr. Treatment / s.c. Immunization
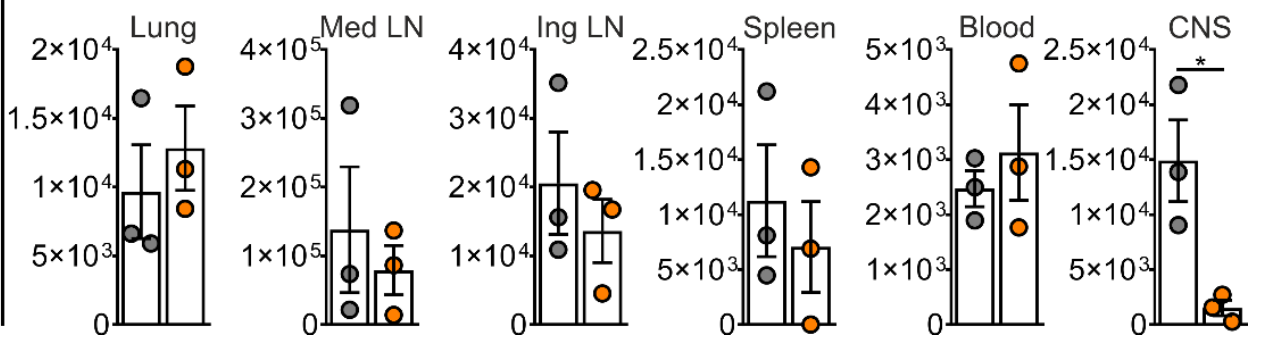

Figure 31: Examination of the relevance of treatment and immunization site for the neomycin effect on $\mathrm{T}_{\mathrm{MBP}}$ cell distribution. Experimental setup and conditions as in Figure 30. On D7 p.i., the distribution of transferred T cells in different organs was determined via flow cytometry. (A) S.c. treatment and i.tr. immunization. (B) S.c. treatment and s.c. immunization. (C) I.tr. treatment and s.c. immunization. $(A-C) T$ cells per $\mathrm{g}$ or $\mathrm{mL}$ detected in the indicated organs on D7 p.i. Values are represented as mean \pm SEM. Statistical significance was determined via unpaired two-tailed $t$-test. $* p<0.05$. 


\subsubsection{Intrapulmonary treatment with neomycin ameliorates passive transfer EAE}

In order to verify the observation that the protective effect of i.tr. neomycin treatment against $E A E$ is independent of the site of induction, we tested the effect of neomycin on ptEAE. Therefore, rats were treated with neomycin or PBS for 7 days and subsequently transferred with in vitro-activated $\mathrm{T}_{\mathrm{MBP} \text {-blast }}$ cells. I.tr. neomycin administration indeed also ameliorated ptEAE (Fig. 32A - E): the incidence of the treated group was reduced to $66.67 \%$ (Fig. 32B). Clinical symptoms started on D5.25 \pm 0.5 p.t.; a significant delay compared to the onset of the PBS group on D4.4 \pm 0.25 p.t. (Fig. 32C). Average peak and cumulative scores of the neomycin group were $0.67 \pm 0.25$ and $1.8 \pm 0.75$, respectively, and thus significantly reduced compared to the corresponding scores of $2.25 \pm 0.38$ and $7.3 \pm 1.18$ of the PBS group (Fig. 32D, E).

These results further strengthen our observation that the neomycin effect on EAE is not due to an impairment in TMBP-EM cell activation.
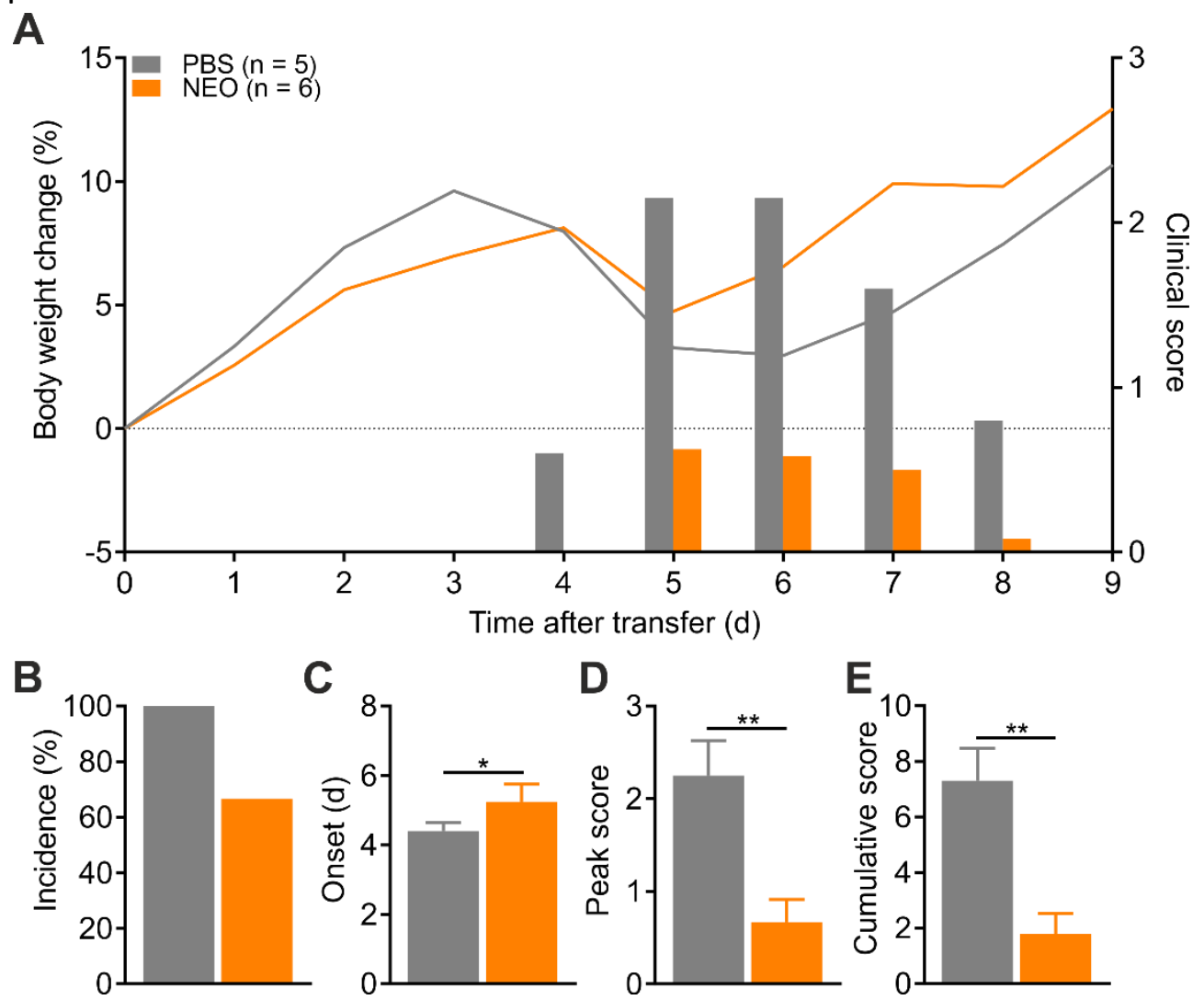

Figure 32: Passive transfer EAE in neomycin-treated rats. Rats were i.tr. treated daily with $1 \mathrm{mg}$ neomycin (NEO) or PBS for

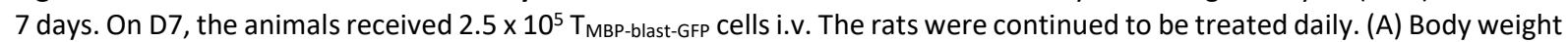
change (lines) and clinical scores (bars) over the course of EAE. (B) Incidence (\%). (C) Average onset (days p.t.). (D) Average peak score. (E) Average cumulative score. Values are represented as mean \pm SEM. Statistical significance was determined via unpaired two-tailed $t$-test. ${ }^{*} p<0.05 ; * *<0.01$. 


\subsubsection{Neomycin treatment does not affect blood-brain barrier integrity and endothelial expression of adhesion molecules}

A recent study found that the gut microbiota contributes to the regulation of BBB permeability (Braniste et al., 2014). In order to test a similar function of the lung microbiota, rats were treated daily with neomycin or PBS for 7 days. Endothelial cells were sorted from meninges and spinal cord parenchyma and the expression of tight junction molecules (claudin-5 and occludin) and adhesion molecules (VCAM-1 and ICAM) was determined.

The expression of all genes examined was similar in the neomycin and the PBS group (Fig. 33A, B).

The data show that neomycin does not influence the expression of genes relevant for BBB permeability or T cell diapedesis in meningeal and parenchymal endothelial cells of the spinal cord.
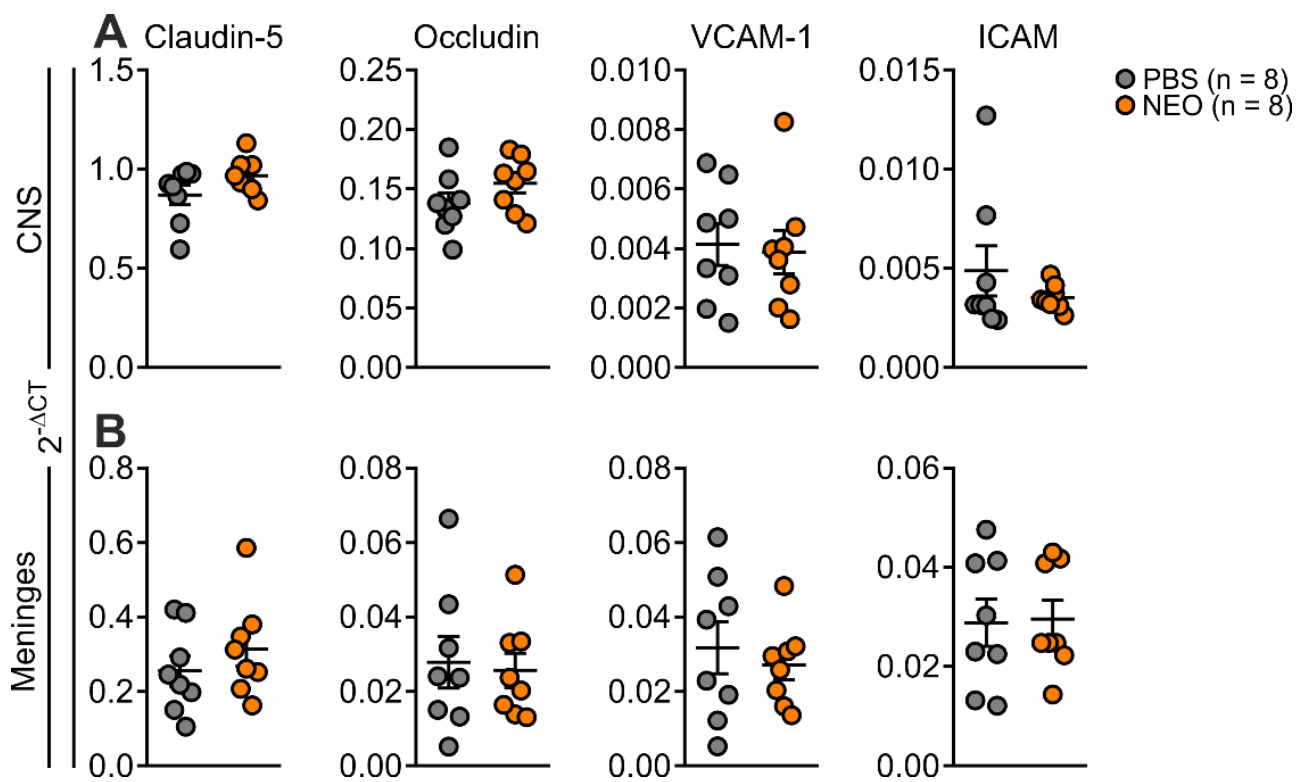

Figure 33: Effect of i.tr. neomycin treatment on BBB integrity and expression of adhesion molecules. Rats were i.tr. treated daily with neomycin (NEO; $1 \mathrm{mg}$ ) or PBS for 7 days. On D7 of treatment, endothelial cells were sorted from spinal cord parenchyma and meninges. Expression of the tight junction genes claudin- 5 and occludin as well as the adhesion molecule genes VCAM-1 and ICAM on mRNA level was determined using qPCR. (A) Gene expression in parenchymal endothelial cells. (B) Gene expression in meningeal endothelial cells. Expression of all genes (2-CCT) was normalized to $\beta$-actin expression of the same samples. Cumulative data from two independent experiments. Values are represented as mean \pm SEM. Statistical significance was determined via unpaired two-tailed $t$-test. 


\subsubsection{Neomycin treatment may affect microglia function}

The gut microbiota might influence the maturation and function of CNS-resident microglia (Erny et al., 2015). We therefore investigated whether the antibiotic treatment of the lung microbiota might be associated with functional impairments of microglia. We did not observe any differences in the expression of TNF $\alpha$, IL- 6 and IL-12 between the neomycin and the PBS group following 7 days of treatment (Fig. 34A - C). However, MHCll expression of the neomycin group was 30.7 \% lower compared to the PBS group (Fig. 34D) suggesting that neomycin treatment could indeed have an effect on microglia or other APCs of the CNS.

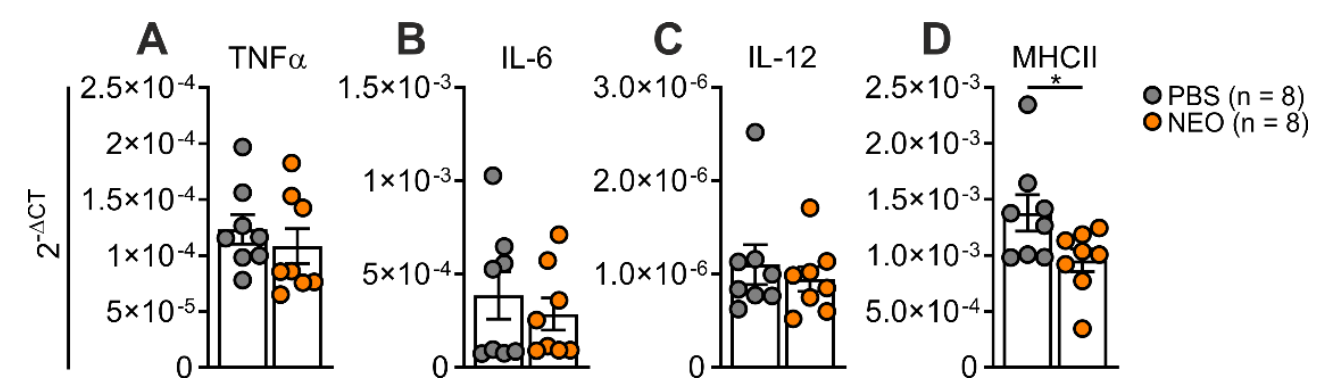

Figure 34: Effect of i.tr. neomycin treatment on microglia-typical gene expression. Rats were i.tr. treated daily with neomycin (NEO; $1 \mathrm{mg}$ ) or PBS for 7 days. On D7 of treatment, the spinal cord parenchyma was isolated and total tissue gene expression of the microglia-typical genes (A) TNF $\alpha,(B)$ IL-6, (C) IL-12 and (D) MHCII on mRNA level was determined using qPCR. Expression of all genes $\left(2^{-\triangle C T}\right)$ was normalized to $\beta$-actin expression of the same samples. Cumulative data from two independent experiments. Values are represented as mean \pm SEM. Statistical significance was determined via unpaired twotailed $t$-test. ${ }^{*} \mathrm{p}<0.05$. 


\section{Discussion}

\subsection{Establishment and characterization of an i.tr. induced EAE model}

The role of the lung as an organ potentially crucial for the initiation of autoimmune diseases has historically not been thoroughly investigated. In a recent study, however, it was observed that adoptively transferred MBP-reactive T cells undergo a functional reprogramming within the lung, thereby equipping the cells with the potential to enter the CNS and induce EAE (Odoardi et al., 2012). Furthermore, the authors could show that neonatally transferred MBPreactive memory $T$ cells that persist in primary lymphatic but also peripheral organs like the lung of the recipients throughout adulthood could be activated by intrapulmonary administration of MBP and CFA to induce EAE (Kawakami et al., 2005; Odoardi et al., 2012).

Based on these findings and in order to better understand the role of the lung in the initiation of CNS autoimmune disease, we established a lung EAE model induced by activation of $T_{M B P}$ EM cells via intrapulmonary immunization. Owing to their retroviral expression of fluorescent markers, the model allows us to track and functionally characterize the disease-causing $\mathrm{T}$ cells, both in the lung and on their way into the CNS.

$T$ cell activation via the lung has classically been proposed to take place in the lung-draining mediastinal lymph nodes: according to this view, antigens expressed by lung pathogens or entering the pulmonary system via inhalation are endocytosed by dendritic cells and transported to the mediastinal lymph nodes where they are presented to T cells in the context of MHCII (Sheridan and Lefrançois, 2011; Caucheteux et al., 2013; Lambrecht et al., 2014). Several studies, however, also report that $\mathrm{T}$ cell activation can already take place within the lung. For instance, it was found that $\mathrm{CCR} 7 \%$ mice exhibit ectopic proliferation of $M$. tuberculosis-specific $\mathrm{CD}^{+} \mathrm{T}$ cells following infection, even though dendritic cell trafficking from lung to mediastinal lymph nodes is defective in these animals (Olmos et al., 2010). Moreover, influenza-specific memory $\mathrm{CD} 4^{+} \mathrm{T}$ cells were found to become reactivated in the lung airways within $24 \mathrm{~h}$ following secondary challenge (Chapman et al., 2011).

In our lung EAE model, $\mathrm{T}_{\mathrm{MBP} \text {-EM }}$ cell activation appears to take place in the lung rather than in the lung-draining lymph nodes. Several lines of evidence support this proposition: (1) EAE can be induced by adoptive transfer of $\mathrm{T}_{\text {MBP-EM }}$ cells stimulated in vitro with APCs isolated from the lung. (2) In vivo i.tr. immunization with LO-OPT or HI antigen concentrations induced $\mathrm{T}_{\mathrm{MBP}-\mathrm{EM}}$ cell activation as measured by the strong up-regulation of IFN $\gamma$ and IL-17 $24 \mathrm{~h}$ p.i. in a dosedependent manner within the lung. (3) Almost no transferred T cells could be detected or retrieved from mediastinal LNs or other peripheral organs at this early time point. (4) Transcriptome analysis performed on transferred $T$ cells isolated from the lung $24 \mathrm{~h}$ p.i. showed a clear shift of the gene expression profile toward proliferation and IFN $\gamma$ signaling. Therefore, at least the initial steps of T cell activation seem to take place within the lung tissue.

Surprisingly when establishing the i.tr. induced EAE model, we observed that antigen doses 75 - 150-fold reduced compared to concentrations used for s.c. immunization induced a severe EAE. Mild EAE could still be induced at a $750-1500$-fold reduced dose. This dose could 
be even further reduced by an additional factor of 10 when using TCR $^{\text {MBP }}$-transgenic $T_{M B P-E M}$ cells.

In the newly established lung EAE model, the number or transferred T cells is relatively high and the interval between transfer and immunization relatively short. As a proof of concept and to rule out a model-dependent artifact effect, we i.tr. immunized a group of memory rats. Similar to the acute model of i.tr. induced EAE, the memory rats developed a severe $E A E$ following i.tr. immunization with very low doses of MBP. Together, these observations demonstrate that the lung is not just capable of but also extremely efficient in inducing CNS autoimmunity.

This observation might also be of relevance in context of CNS autoimmunity in humans: lung infections were found to be correlated with an increased MS risk and are often closely followed by disease relapses (Sibley et al., 1985; Buljevac et al., 2002; Oikonen et al., 2011; Halenius and Hengel, 2014). While a direct associative link remains elusive, molecular mimicry has been suggested as a potential mechanistic basis (Lang et al., 2002; Wucherpfennig and Strominger, 1995). According to this theory, epitopes expressed by lung pathogens and presented to T cells by APCs could structurally resemble self-antigens and consequently result in the activation, proliferation and CNS infiltration of normally dormant autoreactive T cells. It is probable that the amount of such pathogen-derived antigens resembling self-structures present in the lung during infection is quite low. Our observation that highly reduced antigen doses are sufficient and even advantageous over higher doses for $\mathrm{T}_{\text {MBP-EM }}$ cell activation and subsequent EAE induction further underpins the hypothesis that $\mathrm{T}$ cell cross-reactivity with pathogenic antigens may have mechanistic implications in the development and progression of MS.

Additional research, however, would be required in order to further investigate this hypothesis. Examining pathogenicity and functional properties of adoptively transferred $T_{M B P}$ EM cells activated by i.tr. immunization with proteins or peptides resembling MBP to varying degrees and potentially being derived from pathogens may help to better understand the role of molecular mimicry in context of CNS autoimmunity.

Moreover, the identification of niches and lung compartments as well as APC populations important for $\mathrm{T}_{\mathrm{MBP} \text {-EM }}$ cell activation my help shed light on the exact processes that make the lung so particularly efficient in context of EAE initiation.

While titrating the antigen in order to establish the lung EAE model, we observed that high antigen doses (HI) as commonly used for s.c. immunizations were not conducive and even hindering for EAE development: both number and activation of the transferred $T$ cells infiltrating the CNS were highly reduced compared to the LO-OPT group which was in line with a strongly ameliorated disease severity. This observation was unexpected and we next aimed to narrow down a potential underlying mechanism.

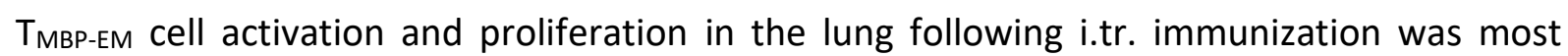
pronounced in animals receiving the $\mathrm{HI}$ concentration of MBP. An enhanced T cell immune response may result in a stronger inflammation of the lung, which may in turn result in the local sequestering and consequent failed recruitment of innate immune cells (e.g. macrophages and neutrophils) to the CNS. This, however, did not appear to be the case: we 
transferred TOVA-EM cells and i.tr. immunized the animals with HI or LO-OPT concentrations of OVA, thus creating a similar inflammatory milieu in the lung as induced by $\mathrm{T}_{\text {MBP-EM }}$ cell transfer and immunization with $\mathrm{HI}$ and LO-OPT doses of MBP. We observed that animals of both groups developed EAE of similar severity following the transfer of $\mathrm{T}_{\text {MBP-blast }}$ cells two days later, thus indirectly excluding an inflammatory state-dependent trapping of myeloid cells in the lung or an impairment in their recruitment to the CNS. In order to further corroborate this observation, however, a quantification of different immune cell populations in lung, blood and CNS over the entire experimental course would need to be conducted.

To test whether the mechanism underlying the observed difference in EAE severity could be ascribed to $T$ cells, transferred $T_{M B P-E M}$ cells that had been activated in the lung by i.tr. immunization with $\mathrm{HI}$ or LO-OPT antigen concentrations were isolated from the spleens of donor animals and retransferred into naïve recipient animals. The antigen dose-dependent difference in i.tr. induced EAE severity could indeed be transferred along with the T cells. Thus, the underlying mechanism is most likely $T$ cell-instrinsic.

An obvious explanation for the antigen dose-dependent difference in i.tr. induced EAE severity

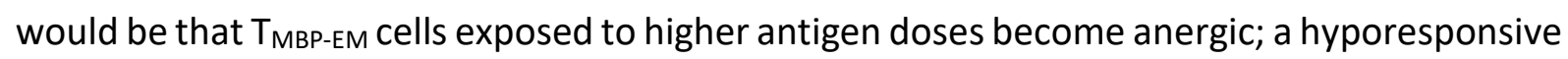
state characterized by a generalized inhibition of proliferation and effector functions. Anergy can broadly be subdivided into two different categories, namely (1) clonal anergy, which describes a growth cycle arrest following weak or incomplete T cell activation and (2) adaptive tolerance, which describes a process of $T$ cell desensitization for their cognate antigen in a situation of persistent antigen abundance following initial activation and proliferation. It often occurs in environments high in co-inhibitory or low in co-stimulatory signals (Schwartz, 2003; Chiodetti et al., 2006; Zheng et al., 2008). A strong activation of TMBP-EM cells in a context of inflammation and antigen persistence may also result in $\mathrm{T}$ cell exhaustion, a process characterized by the stepwise loss of effector functions, which can ultimately result in clonal deletion (Han et al., 2010; Yi et al., 2010; Wherry, 2011). In case of both anergy and exhaustion we would have expected the $\mathrm{T}_{\mathrm{MBP}}$ cells previously activated in the lung with high concentrations of MBP to be impaired in their activation and / or proliferation potential. This was, however, not the case: $T_{\text {MBP }}$ cells isolated from the periphery on D5 p.i. could be activated and proliferated to a similar degree; independent of the antigen dose used for the prior intratracheal immunization. Basic effector functions thus did not appear to be impaired.

It has been reported that $T$ cell clones with higher affinity receptors can be preferentially amplified in conditions of low antigen availability (Rees et al., 1999). Moreover, in an adjuvantbased vaccination study it was observed that low antigen concentrations result in the selective induction of $\mathrm{CD}^{+} \mathrm{T}$ cells with an enhanced functional avidity (Billeskov et al., 2017). We hypothesized that a restricted availability of antigen in the lung might result in the selective expansion of a small subset of particularly encephalitogenic $T_{M B P-E M}$ cell clones whereas higher doses of antigen would result in a broader, non-selective expansion of several clones with varying encephalitogenic potential.

However, TCR reactivities as measured by the ratio of TCR-V $\beta$ chains of previously transferred $T$ cells in the blood of animals i.tr. immunized with $\mathrm{HI}$ and LO-OPT antigen doses were identical. Furthermore, the antigen dose-dependent difference in i.tr. induced EAE severity could also 
be observed when transferring monoclonal $\mathrm{TCR}^{\mathrm{MBP}}$-transgenic $\mathrm{T}$ cells, thereby ruling out a selective $T_{M B P}$ cell subset expansion as a potential mechanism.

It has been shown that adoptively transferred $T_{\text {MBP-blast }}$ cells must undergo a complex reprogramming in peripheral organs in order to gain a migratory profile licensing them to gain access to the CNS and induce EAE (Odoardi et al., 2012). In this newly established lung EAE model, transferred $\mathrm{T}_{\text {MBP-EM }}$ cells undergo similar reprogramming following immunization: antigen-specific $T$ cells in the blood had not only switched off the activation and proliferation program previously induced in the lung by antigenic stimulation, but also additionally acquired a new transcriptional profile characterized by an independent and exclusive regulation of 2579 genes. Based on this observation, we hypothesized that the antigen dose might crucially influence these transcriptional changes. As a consequence, $T_{M B P-E M}$ cells activated in the lung with $\mathrm{HI}$ MBP concentrations could be impaired in their capacity to transgress the blood brain barrier. We thus examined their expression of integrins and chemokine receptors described to be relevant for transendothelial migration.

The integrins VLA-4 and LFA-1 are expressed by a variety of leukocytes and participate in the establishment of cell-cell interactions preceding diapedesis. Both integrins were described to be involved in autoreactive $T$ cell transendothelial migration into the CNS in context of CNS autoimmunity (Bö et al., 1996; Dugger et al., 2009; Elovaara et al., 2000; Sheremata et al., 2005). In the lung EAE model, we observed an upregulation of VLA-4 expression in transferred $\mathrm{T}$ cells sorted from the blood of the $\mathrm{HI}$ and the LO-OPT group before CNS infiltration. This upregulation was, however, of similar extent in both groups. On the contrary, LFA-1 expression was downregulated following i.tr. immunization in the $\mathrm{HI}$ group compared to the NAI control and also tended to be reduced in the LO-OPT group. In the rat TMBP-blast $_{\text {transfer }}$ model commonly used in our lab, interference with VLA-4 signaling was shown to block T cell transgression of the BBB and ameliorate EAE whereas the interference with LFA-1 alone did not affect CNS infiltration or EAE (Bartholomäus et al., 2009). Since the upregulation of VLA-4 expression was similar in the $\mathrm{HI}$ and the LO-OPT group and the expression of LFA-1 if anything downregulated in both groups, we concluded that a differential expression of these integrins can be excluded as a potential mechanism underlying the antigen dose-dependent difference in EAE severity.

Chemokine-signaling induces directed migration and can activate integrin-mediated adhesion of immune cells (Laudanna et al., 2002; Ono et al., 2003): the expression of CXCR3, CXCR4 and CCR5 is upregulated in TMBP cells before CNS infiltration (Odoardi et al., 2012; Schläger et al., 2016). CCR6 signaling was described to be required for Th17 cell infiltration into the CNS via the choroid plexus (Reboldi et al., 2009), a process irrelevant in the rat ptEAE model in which $T_{M B P}$ cells infiltrate the CNS via the leptomeninges (Schläger et al., 2016). Moreover, signaling via CCR5 and CXCR3 determines T cell invasiveness into the CNS by enforcing the adhesiveness of $T_{M B P}$ cells to the leptomeninges. In contrast, CXCR4 signaling in this context appears to be negligible (Schläger et al., 2016).

In the lung EAE model, we observed an increased expression of CXCR3 in all groups compared to the NAI animals by trend. This increase reached significance in the LO-OPT group but was of similar extent in the other groups. No difference in CCR5 and CCR6 expression between the 
groups could be detected. CXCR4 expression was strongly downregulated in the HI group. Reduced expression, however, was also observed in the LO-OPT and the CFA group, though less pronounced.

Since CCR5 and CCR6 expression was not changed and the regulation of CXCR3 and CXCR4 expression tended to be similar in the LO-OPT and the HI group, a differential expression of any of the examined chemokine receptors is unlikely to be causative for the observed effect. Taken together, we did not identify a clear mechanism responsible for the antigen dosedependent difference in EAE severity. It is not unlikely that the mechanism underlying the impaired T cell functionality is multifactorial and owing to a cumulative effect caused by mild differences in the expression of several genes. An in-depth transcriptome analysis of transferred T cells sorted from the blood of the $\mathrm{HI}$ and the LO-OPT group around the time point of CNS invasion may help elucidating if the fundamental and global reprogramming of $\mathrm{T}_{\mathrm{MBP}}$ cells residing in the lung is dysregulated or imbalanced following intrapulmonary activation with a high antigen dose. In addition, identifying and isolating lung and lymph node APCs responsible for the activation of $\mathrm{T}_{\text {MBP-EM }}$ cells in vivo may help establish an in vitro system to functionally compare the relationship between $\mathrm{T}_{\mathrm{MBP} \text {-EM }}$ cells, APCs, microenvironment and antigen dose.

\subsection{Effect of intrapulmonary antibiotic treatment on EAE}

The composition of the gut commensal flora appears to be crucially involved in the regulation of the development of autoimmune diseases (Jangi et al., 2016; Ochoa-Repáraz et al., 2009). Microbiota disruption using antibiotics or raising mice in sterile conditions strongly ameliorates or completely blocks EAE, respectively (Berer et al., 2011; Ochoa-Repáraz et al., 2009). Recently, also the lung was identified to harbor a unique microbiome (Dickson et al., 2013, 2014, 2015). Using our lung EAE model, we aimed to investigate a potential functional connection between the microbiota and CNS autoimmune responses. Therefore, we first determined the natural microbiota composition of lung and gut of Lewis rats, the commonly used experimental animal in our lab, and subsequently manipulated it using antibiotics to evaluate its role in EAE.

In line with previous studies (Dickson et al., 2013, 2014, 2015), we confirmed that the lungs are colonized by a unique microbiota distinct from the gut in quantity and quality: Indeed, the ratio between the gram-negative Bacteriodetes and gram-positive Firmicutes, the most abundant phyla detected in both BALF and feces was significantly different. Moreover, gut and lung also showed fundamental differences in their microbial composition: For instance, the representation of Proteobacteria was much higher in the lung compared to the gut. Fecal samples in contrast showed a higher representation of Cyanobacteria.

We aimed to evaluate the role of the lung microbiota in context of EAE. A combination of the broad-spectrum antibiotics neomycin, vancomycin, ampicillin and metronidazole is commonly used to deplete the gut microbiota in EAE studies (Ochoa-Repáraz et al., 2009; 2010). The latter two antibiotics act systemically and all four are usually administered in relatively high 
doses. This approach results in a drastic and global depletion of the collective microbiota. As alternative approach, EAE studies are often performed in mice raised in germ-free conditions. In these animals, however, the lack of a microbiota leads to several defects in anatomical immune-related structures and impairs the immune response (Luczynski et al., 2016), demonstrating the crucial link between microbiota and the development and function of the immune system (Belkaid and Hand, 2014).

In order to specifically interfere with the lung microbiota without altering the gut microbiota and without systemically affecting the immune system, we decided to treat the animals with a single and locally acting antibiotic. We chose neomycin, a topical broad-spectrum aminoglycoside that mainly but not exclusively targets gram-negative bacteria.

Daily i.tr. neomycin administration of $0.1 \mathrm{mg}, 1 \mathrm{mg}$ and $5 \mathrm{mg}$ for 7 days resulted in changes of the microbiota composition and an increase in $\alpha$-diversity in lung but not gut in a dosedependent manner. Given the relatively low antibiotic dose administered and the incomplete coverage of the neomycin-targeted bacterial spectrum, it is not surprising that the local bacterial community was altered rather than depleted and that $\alpha$-diversity was increased rather than decreased, as rare taxa not affected by antibiotic treatment may increase in abundance and colonize freed up niches in an opportunistic fashion (Price et al., 2017; Lee et al., 2018). Whether i.tr. neomycin administration actually affects the bacterial load of the lung commensal flora remains to be determined based on the expression of tuf genes using qPCR.

We next investigated the effect of i.tr. neomycin treatment on i.tr. induced EAE and observed that T cell infiltration into the CNS and clinical disease were prevented at doses of $1 \mathrm{mg}$ and 5 mg. Protection from EAE was not the consequence of a direct toxic effect of neomycin on $T$ cells nor on their encephalitogenic potential. Moreover, neomycin treatment did not cause a strong lung inflammation that might potentially interfere with the initiation of the CNS autoimmune response: Indeed at $1 \mathrm{mg} /$ day, the minimum effective dose, the immune cell composition of the lung was unaltered compared to PBS-treated control animals. More importantly, the preventive effect of neomycin on EAE was not ascribable to a gut-related effect: $\alpha$-diversity of the gut microbiota was unchanged following i.tr. treatment with neomycin. Moreover, no effects on EAE were observed in animals treated per os with $1 \mathrm{mg}$ or $10 \mathrm{mg}$ of neomycin daily. These data are not in conflict with previous results demonstrating EAE prevention following gut microbiota depletion: Indeed, the protective effect of oral antibiotic treatment against EAE required a combination of several antibiotics administered at doses higher than in our experimental setup (Berer et al., 2011; Ochoa-Repáraz et al., 2009). More importantly, these data strongly support a direct role of the lung microbiota in context of EAE. Recolonization of the lungs of neomycin-treated animals with taxa reduced in abundance upon antibiotic treatment may help understand whether a single or a combination of different taxa could be responsible for the regulation of i.tr. induced EAE.

Over the past few years, the role of symbiotic interactions between microbiota and local environment for the induction, training, and function of the immune system was extensively investigated (Belkaid and Hand, 2014). In the gut, the composition of the local community is crucially involved in the shaping of $\mathrm{CD}^{+} \mathrm{T}$ cell responses. While certain bacteria trigger pro- 
inflammatory responses (Lee et al., 2011; Scher et al., 2013; Wu et al., 2010), others favor the generation of regulatory $T$ cells which in turn participate in the maintenance of gut homeostasis (Coombes et al., 2007; Mucida et al., 2005; Sun et al., 2007). Based on these findings, we asked ourselves whether the neomycin-induced changes in the lung microbiota could affect the nature of the immune response of $T_{M B P-E M}$ cells following their activation. This, however, was not the case: The pro-inflammatory profile of the transferred T cells in the lung $24 \mathrm{~h}$ p.i. was similar between the neomycin- and the PBS-treated group. Moreover, transciptome analysis of the $T$ cells retrieved from the lungs of both groups $24 \mathrm{~h}$ p.i. revealed identical gene expression profiles.

We hypothesized that the observed antibiotic-induced effect on EAE might be independent of intrapulmonary $T$ cell activation. Indeed, i.tr. neomycin treatment ameliorated both active EAE induced by s.c. immunization and passive transfer $E A E$ induced by in vitro activated $T_{M B P}$ blast cells. Independent of the site of immunization, no effect on EAE was observed when the antibiotic was s.c. administered.

While the protective effect of neomycin treatment against EAE appeared to be lung-specific, an altered immune response or impaired $T$ cell activation were widely ruled out as underlying mechanisms, raising the possibility that the lung microbiota may regulate functional crosstalk between lung and CNS through remote control mechanisms.

Recently, it was shown that the gut microbiota influences BBB permeability, supposedly through the release of metabolites like short chain fatty acids (SCFA) into the circulation. An increased BBB permeability was observed in mice raised in germ-free conditions; an effect that could be reversed by fecal transfer from specific-pathogen-free mice (Braniste et al., 2014). To test whether i.tr. neomycin treatment affects BBB properties, we sorted meningeal endothelial cells and determined the expression of the tight junction genes occludin and claudin-5, as well as the adhesion molecules VCAM-1 and ICAM which are the ligands for the integrins VLA-4 and LFA-1, respectively. None of these transcripts was expressed differently between the groups, thus excluding BBB density dysregulation following i.tr. neomycin treatment as a potential mechanism.

The gut commensal flora also appears to be associated with the regulation of microglia: Raising mice in germ-free conditions or depleting their gut microbiota using antibiotics impairs microglia maturation, differentiation and function (Erny et al., 2015). Microglia isolated from the corresponding animals showed impaired effector functions as indicated by a reduced expression of genes including TNF $\alpha$, IL- 6 and IL-12 in response to lipopolysaccharide (LPS) treatment (Erny et al., 2015). Moreover, microglia have been described to represent the main APC population within the CNS parenchyma during neurodegeneration (Schetters et al., 2018). In order to address a potential functional impairment of microglia following i.tr. neomycin treatment, we determined the total tissue expression of the above genes, as well as $\mathrm{MHClI}$, in CNS parenchyma isolated from neomycin-treated and non-treated animals. While neither TNF $\alpha$ nor IL- 6 or IL-12 expression was altered, we observed a lower expression of $\mathrm{MHCll}$ in the neomycin group. How this observation translates into a functional impairment in the antigen presentation capacity of microglia or other APCs of the CNS and how this effect is mediated, however, remains to be determined. 
Taken together, these data strongly support the existence of a lung-CNS axis that can influence CNS autoimmunity independent of the gut-CNS axis. Further research is required in order to understand the mechanistic aspects and identify cellular and metabolic players involved in and required for the mutually regulatory interconnection between lung, microbiota and CNS in context of autoimmune disease. 


\section{References}

Andersen KS, Kirkegaard RH, Karst SM, Albertsen M (2018). Ampvis2: An R Package to Analyse and Visualise 16S RRNA Amplicon Data. BioRxiv 299537.

Antonovsky A, Leibowitz U, Smith HA, Medalie JM, Balogh M, Kats R, Halpern L, Alter M (1965). Epidemiologic Study of Multiple Sclerosis in Israel. I. an Overall Review of Methods and Findings. Archives of Neurology 13:183-93.

Ascherio A (2013). Environmental Factors in Multiple Sclerosis. Expert Review of Neurotherapeutics 13(Suppl2):3-9.

Ascherio A, Munger KL (2007a). Environmental Risk Factors for Multiple Sclerosis. Part I: The Role of Infection. Annals of Neurology 61(4):288-99.

Ascherio A, Munger KL (2007b). Environmental Risk Factors for Multiple Sclerosis. Part II: Noninfectious Factors. Annals of Neurology 61(6):504-13.

Ascherio A, Munger KL, Simon KC (2010). Vitamin D and Multiple Sclerosis. The Lancet Neurology 9(6):599-612.

Baecher-Allan C, Kaskow BJ, Weiner HL (2018). Multiple Sclerosis: Mechanisms and Immunotherapy. Neuron 97(4):742-68.

Baranzini SE (2011). Revealing the Genetic Basis of Multiple Sclerosis: Are We There Yet? Current Opinion in Genetics and Development 21(3):317-24.

Bartholomäus I, Kawakami N, Odoardi F, Schläger C, Miljkovic D, Ellwart JW, Klinkert WEF, Flügel-Koch C, Issekutz TB, Wekerle H, Flügel A (2009). Effector T Cell Interactions with Meningeal Vascular Structures in Nascent Autoimmune CNS Lesions. Nature 462(7269):94-98.

Belkaid Y, Hand TW (2014). Role of the Microbiota in Immunity and Inflammation. Cell 157(1):121-41.

Ben-Nun A, Wekerle H, Cohen IR (1981). The Rapid Isolation of Clonable Antigen-specific T Lymphocyte Lines Capable of Mediating Autoimmune Encephalomyelitis. European Journal of Immunology 11(3):195-99.

Berer K, Mues M, Koutrolos M, Rasbi ZA, Boziki M, Johner C, Wekerle H, Krishnamoorthy G (2011). Commensal Microbiota and Myelin Autoantigen Cooperate to Trigger Autoimmune Demyelination. Nature 479(7374):538-41.

Billeskov R, Wang Y, Solaymani-Mohammadi S, Frey B, Kulkarni S, Andersen P, Agger EM, Sui Y, Berzofsky JA (2017). Low Antigen Dose in Adjuvant-Based Vaccination Selectively Induces CD4 T Cells with Enhanced Functional Avidity and Protective Efficacy. The Journal of Immunology 198(9):3494-3506.

Bö L, Peterson JW, Mørk SJ, Hoffman PA, Gallatin WM, Ransohoff RM, Trapp BD (1996). Distribution of Immunoglobulin Superfamily Members ICAM-1, $-2,-3$, and the Beta 2 Integrin LFA-1 in Multiple Sclerosis Lesions. Journal of Neuropathology and Experimental Neurology 55(10):1060-72.

Braniste V, Al-Asmakh M, Kowal C, Anuar F, Abbaspour A, Tóth M, Korecka A, Bakocevic N, Ng LG, Kundu P, Gulyás B, Halldin C, Hultenby K, Nilsson H, Hebert H, Volpe BT, Diamond B, Pettersson $S$ (2014). The Gut Microbiota Influences Blood-Brain Barrier Permeability in Mice. Science Translational Medicine 6(263):263ra158.

Budhram A, Parvathy S, Kremenchutzky M, Silverman M (2017). Breaking down the Gut Microbiome 
Composition in Multiple Sclerosis. Multiple Sclerosis Journal 23(5):628-36.

Buljevac D, Flach HZ, Hop WCJ, Hijdra D, Laman JD, Savelkoul HFJ, van Der Meché FGA, van Doorn PA, Hintzen RQ (2002). Prospective Study on the Relationship between Infections and Multiple Sclerosis Exacerbations. Brain : A Journal of Neurology 125(Pt 5):952-60.

Caucheteux SM, Torabi-Parizi P, Paul WE (2013). Analysis of Naive Lung CD4 T Cells Provides Evidence of Functional Lung to Lymph Node Migration. Proceedings of the National Academy of Sciences 110(5):1821-26.

Chapman TJ, Lambert K, Topham DJ (2011). Rapid Reactivation of Extralymphoid CD4 T Cells during Secondary Infection. PLOS ONE 6(5):e20493.

Charlson ES, Chen J, Custers-Allen R, Bittinger K, Li H, Sinha R, Hwang J, Bushman FD, Collman RG (2010). Disordered Microbial Communities in the Upper Respiratory Tract of Cigarette Smokers. PLOS ONE 5(12):e15216.

Chen S, Zhou Y, Chen Y, Gu J (2018). Fastp: An Ultra-Fast All-in-One FASTQ Preprocessor. Bioinformatics 34(17):i884-90

Chiodetti L, Choi S, Barber DL, Schwartz RH (2006). Adaptive Tolerance and Clonal Anergy Are Distinct Biochemical States. The Journal of Immunology 176(4):2279-91.

Chu F, Shi M, Lang Y, Shen D, Jin T, Zhu J, Cui L (2018). Gut Microbiota in Multiple Sclerosis and Experimental Autoimmune Encephalomyelitis: Current Applications and Future Perspectives. Mediators of Inflammation 2018:1-17.

Compston A, McDonald I, Noseworthy J, Lassmann H, Miller D, Smith K, Wekerle H, Confavreux C (2006). McAlpine's Multiple Sclerosis. Churchill Livingstone, Elsevier.

Compston A, Coles A (2002). Multiple Sclerosis. The Lancet 359(9313):1221-31.

Compston A, Coles A (2008). Multiple Sclerosis. The Lancet 372(9648):1502-17.

Coombes JL, Siddiqui KR, Arancibia-Cárcamo CV, Hall J, Sun CM, Belkaid Y, Powrie F (2007). A Functionally Specialized Population of Mucosal CD103+ DCs Induces Foxp3+ Regulatory T Cells via a TGF-Beta and Retinoic Acid-Dependent Mechanism. The Journal of Experimental Medicine 204(8):1757-64.

Correale J, Fiol M, Gilmore W (2006). The Risk of Relapses in Multiple Sclerosis during Systemic Infections. Neurology 67(4):652-59.

Dang AT, Marsland BJ (2019). Microbes, Metabolites, and the Gut-Lung Axis. Mucosal Immunology Jul;12(4):843-850.

Dickson RP, Erb-Downward JR, Huffnagle GB (2013). The Role of the Bacterial Microbiome in Lung Disease. Expert Review of Respiratory Medicine 7(3):245-57.

Dickson RP, Huffnagle GB (2015). The Lung Microbiome: New Principles for Respiratory Bacteriology in Health and Disease. PLOS Pathogens 11(7):e1004923.

Dickson RP, Martinez FJ, Huffnagle GB (2014). The Role of the Microbiome in Exacerbations of Chronic Lung Diseases. The Lancet 384(9944):691-702.

Disanto G, Morahan JM, Barnett MH, Giovannoni G, Ramagopalan SV (2012). The Evidence for a Role of B Cells in Multiple Sclerosis. Neurology 78(11):823-32. 
Dugger KJ, Zinn KR, Weaver C, Bullard DC, Barnum SR (2009). Effector and Suppressor Roles for LFA-1 during the Development of Experimental Autoimmune Encephalomyelitis. Journal of Neuroimmunology 206(1-2):22-27.

Ebers GC, Yee IM, Sadovnick AD, Duquette P (2000). Conjugal Multiple Sclerosis: Population-Based Prevalence and Recurrence Risks in Offspring. Canadian Collaborative Study Group. Annals of Neurology 48(6):927-31.

Egesten A, Brandt L, Olsson T, Granath F, Inghammar M, Löfdahl CG, Ekbom A (2008). Increased Prevalence of Multiple Sclerosis among COPD Patients and Their First-Degree Relatives: A Population-Based Study. Lung 186(3):173-78.

Elovaara I, Ukkonen M, Leppäkynnäs M, Lehtimäki T, Luomala M, Peltola J, Dastidar P (2000). Adhesion Molecules in Multiple Sclerosis: Relation to Subtypes of Disease and Methylprednisolone Therapy. Archives of Neurology 57(4):546-51.

Erny D, Hrabe De Angelis AL, Jaitin D, Wieghofer P, Staszewski O, David E, Keren-Shaul H, Mahlakoiv T, Jakobshagen K, Buch T, Schwierzeck V, Utermöhlen O, Chun E, Garrett WS, Mccoy KD, Diefenbach A, Staeheli P, Stecher B, Amit I, Prinz M (2015). Host Microbiota Constantly Control Maturation and Function of Microglia in the CNS. Nature Neuroscience 18(7):965-77.

Flügel A, Willem M, Berkowicz T, Wekerle H (1999). Gene Transfer into CD4+ T Lymphocytes: Green Fluorescent Protein- Engineered, Encephalitogenic T Cells Illuminate Brain Autoimmune Responses. Nature Medicine 5(7):843-47.

Ghadirian P, Dadgostar B, Azani R, Maisonneuve P (2001). A Case-Control Study of the Association between Socio-Demographic, Lifestyle and Medical History Factors and Multiple Sclerosis. Canadian Journal of Public Health 92(4):281-85.

Goverman J, Woods A, Larson L, Weiner LP, Hood L, Zaller DM (1993). Transgenic Mice That Express a Myelin Basic Protein-Specific T Cell Receptor Develop Spontaneous Autoimmunity. Cell 72(4):551-60.

Halenius A, Hengel H (2014). Human Cytomegalovirus and Autoimmune Disease. BioMed Research International 2014:1-15.

Han S, Asoyan A, Rabenstein H, Nakano N, Reinhard Obst R (2010). Role of Antigen Persistence and Dose for CD4+ T-Cell Exhaustion and Recovery. Proceedings of the National Academy of Sciences 107(47):20453-58.

Hawkes CH (2007). Smoking Is a Risk Factor for Multiple Sclerosis: A Metanalysis. Multiple Sclerosis 13(5):610-15.

Hedström AK, Bäärnhielm M, Olsson T, Alfredsson L (2009). Tobacco Smoking, but Not Swedish Snuff Use, Increases the Risk of Multiple Sclerosis. Neurology 73(9):696-701.

Hedström AK, Hillert J, Olsson T, Alfredsson L (2013). Smoking and Multiple Sclerosis Susceptibility. European Journal of Epidemiology 28(11):867-74.

Hemmer B, Archelos JJ, Hartung HP (2002). New Concepts in the Immunopathogenesis of Multiple Sclerosis. Nature Reviews Neuroscience 3(4):291-301.

Hernán MA, Olek MJ, Ascherio A (2001). Cigarette Smoking and Incidence of Multiple Sclerosis. American Journal of Epidemiology 154(1):69-74.

Hilty M, Burke C, Pedro H, Cardenas P, Bush A, Bossley C, Davies J, Ervine A, Poulter L, Pachter L, Moffatt MF, Cookson WO (2010). Disordered Microbial Communities in Asthmatic Airways. PLoS ONE 
$5(1): e 8578$.

International Multiple Sclerosis Genetics Consortium; Wellcome Trust Case Control Consortium 2, Sawcer S, Hellenthal G, Pirinen M, Spencer CC, Patsopoulos NA, Moutsianas L, Dilthey A, Su Z, Freeman C, Hunt SE, Edkins S, Gray E, Booth DR, Potter SC, Goris A, Band G, Oturai AB, Strange A, Saarela J, Bellenguez C, Fontaine B, Gillman M, Hemmer B, Gwilliam R, Zipp F, Jayakumar A, Martin R, Leslie S, Hawkins S, Giannoulatou E, D'alfonso S, Blackburn H, Martinelli Boneschi F, Liddle J, Harbo HF, Perez ML, Spurkland A, Waller MJ, Mycko MP, Ricketts M, Comabella M, Hammond N, Kockum I, McCann OT, Ban M, Whittaker P, Kemppinen A, Weston P, Hawkins C, Widaa S, Zajicek J, Dronov S, Robertson N, Bumpstead SJ, Barcellos LF, Ravindrarajah R, Abraham R, Alfredsson L, Ardlie K, Aubin C, Baker A, Baker K, Baranzini SE, Bergamaschi L, Bergamaschi R, Bernstein A, Berthele A, Boggild M, Bradfield JP, Brassat D, Broadley SA, Buck D, Butzkueven $H$, Capra R, Carroll WM, Cavalla P, Celius EG, Cepok S, Chiavacci R, Clerget-Darpoux F, Clysters K, Comi G, Cossburn M, Cournu-Rebeix I, Cox MB, Cozen W, Cree BA, Cross AH, Cusi D, Daly MJ, Davis E, de Bakker PI, Debouverie M, D'hooghe MB, Dixon K, Dobosi R, Dubois B, Ellinghaus D, Elovaara I, Esposito F, Fontenille C, Foote S, Franke A, Galimberti D, Ghezzi A, Glessner J, Gomez R, Gout O, Graham C, Grant SF, Guerini FR, Hakonarson H, Hall P, Hamsten A, Hartung HP, Heard RN, Heath $S$, Hobart J, Hoshi $M$, Infante-Duarte $C$, Ingram $G$, Ingram $W$, Islam $T$, Jagodic $M$, Kabesch M, Kermode AG, Kilpatrick TJ, Kim C, Klopp N, Koivisto K, Larsson M, Lathrop M, LechnerScott JS, Leone MA, Leppä V, LiljedahI U, Bomfim IL, Lincoln RR, Link J, Liu J, Lorentzen AR, Lupoli S, Macciardi F, Mack T, Marriott M, Martinelli V, Mason D, McCauley JL, Mentch F, Mero IL, Mihalova T, Montalban X, Mottershead J, Myhr KM, Naldi P, Ollier W, Page A, Palotie A, Pelletier J, Piccio L, Pickersgill T, Piehl F, Pobywajlo S, Quach HL, Ramsay PP, Reunanen M, Reynolds R, Rioux JD, Rodegher M, Roesner S, Rubio JP, Rückert IM, Salvetti M, Salvi E, Santaniello A, Schaefer CA, Schreiber S, Schulze C, Scott RJ, Sellebjerg F, Selmaj KW, Sexton D, Shen L, Simms-Acuna B, Skidmore S, Sleiman PM, Smestad C, Sørensen PS, Søndergaard HB, Stankovich J, Strange RC, Sulonen AM, Sundqvist E, Syvänen AC, Taddeo F, Taylor B, Blackwell JM, Tienari P, Bramon E, Tourbah A, Brown MA, Tronczynska E, Casas JP, Tubridy N, Corvin A, Vickery J, Jankowski J, Villoslada P, Markus HS, Wang K, Mathew CG, Wason J, Palmer CN, Wichmann HE, Plomin R, Willoughby E, Rautanen A, Winkelmann J, Wittig M, Trembath RC, Yaouanq J, Viswanathan AC, Zhang H, Wood NW, Zuvich R, Deloukas P, Langford C, Duncanson A, Oksenberg JR, Pericak-Vance MA, Haines JL, Olsson T, Hillert J, Ivinson AJ, De Jager PL, Peltonen L, Stewart GJ, Hafler DA, Hauser SL, McVean G, Donnelly P, Compston A (2011). Genetic Risk and a Primary Role for Cell-Mediated Immune Mechanisms in Multiple Sclerosis. Nature 476(7359):214-19.

Jafari N, Hintzen RQ (2011). The Association between Cigarette Smoking and Multiple Sclerosis. Journal of the Neurological Sciences 311(1-2):78-85.

Janda JM, Abbott SL (2007). 16S RRNA Gene Sequencing for Bacterial Identification in the Diagnostic Laboratory: Pluses, Perils, and Pitfalls. Journal of Clinical Microbiology 45(9):2761-64.

Jangi S, Gandhi R, Cox LM, Li N, Von Glehn F, Yan R, Patel B, Mazzola MA, Liu S, Glanz BL, Cook S, Tankou S, Stuart F, Melo K, Nejad P, Smith K, Topçuolu BD, Holden J, Kivisäkk P, Chitnis T, De Jager PL, Quintana FJ, Gerber GK, Bry L, Weiner HL (2016). Alterations of the Human Gut Microbiome in Multiple Sclerosis. Nature Communications 7(1):12015.

Joscelyn J, Kasper LH (2014). Digesting the Emerging Role for the Gut Microbiome in Central Nervous System Demyelination. Multiple Sclerosis Journal 20(12):1553-59.

Kawakami N, Odoardi F, Ziemssen T, Bradl M, Ritter T, Neuhaus O, Lassmann H, Wekerle H, Flügel A (2005). Autoimmune CD4+ T Cell Memory: Lifelong Persistence of Encephalitogenic T Cell Clones in Healthy Immune Repertoires. Journal of Immunology 175(1):69-81.

Kebir H, Kreymborg K, Ifergan I, Dodelet-Devillers A, Cayrol R, Bernard M, Giuliani F, Arbour N, Becher 
B, Prat A (2007). Human TH17 Lymphocytes Promote Blood-Brain Barrier Disruption and Central Nervous System Inflammation. Nature Medicine 13(10):1173-75.

Kitz A (2013). Generation and Analysis of T Cell Receptor Transgenic Rats to Model CNS Autoimmunity.

Klindworth A, Pruesse E, Schweer T, Peplies J, Quast C, Horn M, Glöckner FO (2013). Evaluation of General 16S Ribosomal RNA Gene PCR Primers for Classical and Next-Generation SequencingBased Diversity Studies. Nucleic Acids Research 41(1):e1.

Lambrecht BN, Pauwels RA, Fazekas de St. Groth B (2014). Induction of Rapid T Cell Activation, Division, and Recirculation by Intratracheal Injection of Dendritic Cells in a TCR Transgenic Model. Journal of Immunology 164(6):2937-46.

Lang L, Jacobsen H, Ikemizu S, Andersson C, Harlos K, Madsen L, Hjorth P, Sondergaard L, Svejgaard A, Wucherpfennig K, Stuart DI, Bell JI, Jones EY, Fugger L (2002). A Functional and Structural Basis for TCR Cross-Reactivity in Multiple Sclerosis. Nature Immunology 3(10):940-43.

Laudanna C, Kim JY, Constantin G, Butcher E (2002). Rapid Leukocyte Integrin Activation by Chemokines. Immunological Reviews 186:37-46.

Lee L, Savage VM, eh PJ (2018). Intermediate Levels of Antibiotics May Increase Diversity of Colony Size Phenotype in Bacteria. Computational and Structural Biotechnology Journal 16:307-15.

Lee YK, Menezes JS, Umesaki Y, Mazmanian SK (2011). Proinflammatory T-Cell Responses to Gut Microbiota Promote Experimental Autoimmune Encephalomyelitis. Proceedings of the National Academy of Sciences 108(Suppl1):4615-22.

Lipton MM, Freund J (1953). Allergic Encephalomyelitis in the Rat Induced by the Intracutaneous Injection of Central Nervous System Tissue and Adjuvants. Journal of Immunology 71(2):98-109.

Lublin FD, Reingold SC (1996). Defining the Clinical Course of Multiple Sclerosis: Results of an International Survey. National Multiple Sclerosis Society (USA) Advisory Committee on Clinical Trials of New Agents in Multiple Sclerosis. Neurology 46(4):907-11.

Luczynski P, Mc Vey Neufeld KA, Oriach CS, Clarke G, Dinan TG, Cryan JF (2016). Growing up in a Bubble: Using Germ-Free Animals to Assess the Influence of the Gut Microbiota on Brain and Behavior. International Journal of Neuropsychopharmacology 19(8):1-17.

Mannie M, Swanborg RH, Stepaniak JA (2009). Experimental Autoimmune Encephalomyelitis in the Rat. Current Protocols in Immunology 85(1):15.2.1-15.2.15.

Martin M (2011). Cutadapt Removes Adapter Sequences from High-Throughput Sequencing Reads. EMBnet.Journal 17(1):10.

Miller D, Barkhof F, Montalban X, Thompson A, Filippi M (2005). Clinically Isolated Syndromes Suggestive of Multiple Sclerosis, Part I: Natural History, Pathogenesis, Diagnosis, and Prognosis. The Lancet Neurology 4(5):281-88.

Miller DH, Leary SM (2007). Primary-Progressive Multiple Sclerosis. Lancet Neurology 6(10):903-12.

Miller SD, Karpus WJ, Davidson TS (2010). Experimental Autoimmune Encephalomyelitis in the Mouse. Current Protocols in Immunology 88(1):15.1.1-15.1.20.

Milo R, Kahana E (2010). Multiple Sclerosis: Geoepidemiology, Genetics and the Environment. Autoimmunity Reviews 9(5):A387-94.

Mix E, Meyer-Rienecker H, Hartung HP, Zettl UK (2010). Animal Models of Multiple Sclerosis-Potentials 
and Limitations. Progress in Neurobiology 92(3):386-404.

Mucida D, Kutchukhidze N, Erazo A, Russo M, Lafaille JJ, Curotto De Lafaille MA (2005). Oral Tolerance in the Absence of Naturally Occurring Tregs. Journal of Clinical Investigation 115(7):1923-33.

O'Brien K, Gran B, Rostami A (2010). T-Cell Based Immunotherapy in Experimental Autoimmune Encephalomyelitis and Multiple Sclerosis. Immunotherapy 2(1):99-115.

Ochoa-Repáraz J, Mielcarz DW, Ditrio LE, Burroughs AR, Foureau DM, Haque-Begum S, Kasper LH (2009). Role of Gut Commensal Microflora in the Development of Experimental Autoimmune Encephalomyelitis. Journal of Immunology (Baltimore, Md. : 1950) 183(10):6041-50.

Ochoa-Repáraz J, Mielcarz DW, Haque-Begum S, Kasper LH (2010). Induction of a Regulatory B Cell Population in Experimental Allergic Encephalomyelitis by Alteration of the Gut Commensal Microflora. Gut Microbes 1(2):103-8.

Odoardi F, Sie C, Streyl K, Ulaganathan VJ, Schläger C, Lodygin D, Heckelsmiller K, Nietfeld W, Ellwart J, Klinkert WE, Lottaz C, Nosov M, Brinkmann V, Spang R, Lehrach H, Vingron M, Wekerle H, FlügelKoch C, Flügel A (2012). T Cells Become Licensed in the Lung to Enter the Central Nervous System. Nature 488(7413):675-79.

Oikonen M, Laaksonen M, Aalto V, Ilonen J, Salonen R, Erälinna JP, Panelius M, Salmi A (2011). Temporal Relationship between Environmental Influenza A and Epstein-Barr Viral Infections and High Multiple Sclerosis Relapse Occurrence. Multiple Sclerosis Journal 17(6):672-80.

Olmos S, Stukes S, Ernst JD (2010). Ectopic Activation of Mycobacterium Tuberculosis-Specific CD4+ T Cells in Lungs of CCR7-/- Mice. The Journal of Immunology 184(2):895-901.

Ono SJ, Nakamura T, Miyazaki D, Ohbayashi M, Dawson M, Toda M (2003). Chemokines: Roles in Leukocyte Development, Trafficking, and Effector Function. The Journal of Allergy and Clinical Immunology 111(6):1185-99.

Panitch HS (1994). Influence of Infection on Exacerbations of Multiple Sclerosis. Annals of Neurology 36 Suppl:S25-8.

Parashar A, Udayabanu M (2017). Gut Microbiota: Implications in Parkinson's Disease. Parkinsonism and Related Disorders 38:1-7.

Paterson PY (1960). Transfer of Allergic Encephalomyelitis in Rats by Means of Lymph Node Cells. The Journal of Experimental Medicine 111:119-36.

Pekmezovic T, Drulovic J, Milenkovic M, Jarebinski M, Stojsavljevic N, Mesaros S, Kisic D, Kostic J (2006). Lifestyle Factors and Multiple Sclerosis: A Case-Control Study in Belgrade. Neuroepidemiology 27(4):212-16.

Price LB, Hungate BA, Koch BJ, Davis GS, Liu CM (2017). Colonizing Opportunistic Pathogens (COPs): The Beasts in All of Us. PLoS Pathogens 13(8):e1006369.

Ransohoff RM (2012). Licensed in the Lungs. Nature 488:595.

Reboldi A, Coisne C, Baumjohann D, Benvenuto F, Bottinelli D, Lira S, Uccelli A, Lanzavecchia A, Engelhardt B, Sallusto F (2009). C-C Chemokine Receptor 6-Regulated Entry of TH-17 Cells into the CNS through the Choroid Plexus Is Required for the Initiation of EAE. Nature Immunology 10(5):514-23.

Rees W, Bender J, Teague TK, Kedl RM, Crawford F, Marrack P, Kappler J (1999). An Inverse Relationship between T Cell Receptor Affinity and Antigen Dose during CD4+ T Cell Responses in Vivo and in 
Vitro. Proceedings of the National Academy of Sciences 96(17):9781-86.

Riise T, Nortvedt MW, Ascherio A (2003). Smoking Is a Risk Factor for Multiple Sclerosis. Neurology 61(8):1122-24.

Rivers TM, Sprunt DH, Berry GP (1933). Observations on Attempts to Produce Acute Disseminated Encephalomyelitis in Monkeys. The Journal of Experimental Medicine 58(1):39-53.

Robertson NP, Fraser M, Deans J, Clayton D, Walker N, Compston DA (1996). Age-Adjusted Recurrence Risks for Relatives of Patients with Multiple Sclerosis. Brain 119(2):449-55.

Rognes, Torbjørn R, Flouri T, Nichols B, Quince C, Mahé F (2016). VSEARCH: A Versatile Open Source Tool for Metagenomics. PeerJ 4:e2584.

Scales BS, Dickson RP, Huffnagle GB (2016). A Tale of Two Sites: How Inflammation Can Reshape the Microbiomes of the Gut and Lungs. Journal of Leukocyte Biology 100(5):943-50.

Scher JU, Sczesnak A, Longman RS, Segata N, Ubeda C, Bielski C, Rostron T, Cerundolo V, Pamer EG, Abramson SB, Huttenhower C, Littman DR (2013). Expansion of Intestinal Prevotella Copri Correlates with Enhanced Susceptibility to Arthritis. ELife 2:e01202.

Schetters ST, Gomez-Nicola D, Garcia-Vallejo JJ, Van Kooyk Y (2018). Neuroinflammation: Microglia and T Cells Get Ready to Tango. Frontiers in Immunology 8:1905.

Schläger C, Körner H, Krueger M, Vidoli S, Haberl M, Mielke D, Brylla E, Issekutz T, Cabanãs C, Nelson PJ, Ziemssen T, Rohde V, Bechmann I, Lodygin D, Odoardi F, Flügel A (2016). Effector T-Cell Trafficking between the Leptomeninges and the Cerebrospinal Fluid. Nature 530(7590):349-53.

Schwartz RH (2003). T Cell Anergy. Annual Review of Immunology 21(1):305-34.

Sheremata WA, Minagar A, Alexander JS, Vollmer T (2005). The Role of Alpha-4 Integrin in the Aetiology of Multiple Sclerosis: Current Knowledge and Therapeutic Implications. CNS Drugs 19(11):909-22.

Sheridan BS, Lefrançois L (2011). Regional and Mucosal Memory T Cells. Nature Immunology 12(6):485-91.

Shi Y, Feng Y, Kang J, Liu C, Li Z, Li D, Cao W, Qiu J, Guo Z, Bi E, Zang L, Lu C, Zhang JZ, Pei G (2007). Critical Regulation of $\mathrm{CD} 4+\mathrm{T}$ Cell Survival and Autoimmunity by $\beta$-Arrestin 1. Nature Immunology 8(8):817-24.

Sibley WA, Bamford CR, Clark K (1985). Clinical Viral Infections and Multiple Sclerosis. The Lancet 1(8441):1313-15.

Sun CM, Hall JA, Blank RB, Bouladoux N, Oukka M, Mora JR, Belkaid Y (2007). Small Intestine Lamina Propria Dendritic Cells Promote de Novo Generation of Foxp3 T Reg Cells via Retinoic Acid. The Journal of Experimental Medicine 204(8):1775-85.

Sundström P, Nyström L (2008). Smoking Worsens the Prognosis in Multiple Sclerosis. Multiple Sclerosis 14(8):1031-35.

Tzartos JS, Friese MA, Craner MJ, Palace J, Newcombe J, Esiri MM, Fugger L (2008). Interleukin-17 Production in Central Nervous System-Infiltrating T Cells and Glial Cells Is Associated with Active Disease in Multiple Sclerosis. American Journal of Pathology 172(1):146-55.

Viglietta V, Baecher-Allan C, Weiner HL, Hafler DA (2004). Loss of Functional Suppression by CD4 + CD25 + Regulatory T Cells in Patients with Multiple Sclerosis. The Journal of Experimental 
Medicine 199(7):971-79.

Weinshenker BG, Bass B, Rice GP, Noseworthy J, Carriere W, Baskerville J, Ebers GC (1989). The Natural History of Multiple Sclerosis: A Geographically Based Study. I. Clinical Course and Disability. Brain : A Journal of Neurology 112:133-46.

Westfall S, Lomis N, Kahouli I, Dia SY, Singh SP, Prakash S (2017). Microbiome, Probiotics and Neurodegenerative Diseases: Deciphering the Gut Brain Axis. Cellular and Molecular Life Sciences 74(20):3769-87.

Wherry EJ (2011). T Cell Exhaustion. Nature Immunology 12(6):492-99.

Wickham H (2011). Ggplot2. Wiley Interdisciplinary Reviews: Computational Statistics 3(2):180-85.

World Health Organization (2008). Atlas: Multiple Sclerosis Resources in the World 2008. Geneva: World Health Organization 15-16.

Wu HJ, Ivanov II, Darce J, Hattori K, Shima T, Umesaki Y, Littman DR, Benoist C, Mathis D (2010). GutResiding Segmented Filamentous Bacteria Drive Autoimmune Arthritis via T Helper 17 Cells. Immunity 32(6):815-27.

Wucherpfennig KW, Strominger JL (1995). Molecular Mimicry in T Cell-Mediated Autoimmunity: Viral Peptides Activate Human T Cell Clones Specific for Myelin Basic Protein. Cell 80(5):695-705.

Yi JS, Cox MA, Zajac AJ (2010). T-Cell Exhaustion: Characteristics, Causes and Conversion. Immunology 129(4):474-81.

Zhang J, Kobert K, Flouri T, Stamatakis A (2014). PEAR: A Fast and Accurate Illumina Paired-End ReAd MergeR. Bioinformatics 30(5):614-20.

Zhang X, Zhang D, Jia H, Feng Q, Wang D, Liang D, Wu X, Li J, Tang L, Li Y, Lan Z, Chen B, Li Y, Zhong H, Xie H, Jie Z, Chen W, Tang S, Xu X, Wang X, Cai X, Liu S, Xia Y, Li J, Qiao X, Al-Aama JY, Chen H, Wang L, Wu QJ, Fengchun Zhang F, Wenjie Zheng W, Yongzhe Li Y, Mingrong Zhang M, Luo G, Xue W, Xiao L, Li J, Chen W, Xu X, Yin Y, Yang H, Wang J, Kristiansen K, Liu L, Li T, Huang Q, Li Y, Wang $J$ (2015). The Oral and Gut Microbiomes Are Perturbed in Rheumatoid Arthritis and Partly Normalized after Treatment. Nature Medicine 21(8):895-905.

Zheng Y, Zha Y, Gajewski TF (2008). Molecular Regulation of T-Cell Anergy. EMBO Reports 9(1):50-55. 


\section{Acknowledgments}

First of all, I would like to thank Prof. Alexander Flügel for his support, advice and guidance during my doctoral studies and for giving me the opportunity to work at his institute.

Secondly, I want to thank Dr. Francesca Odoardi for her amazing supervision, her dedication and patience, and for her continuous help and encouragement.

Furthermore, I would like to thank Prof. Holger Reichardt and Prof. Wolfgang Brück for being part of my thesis advisory committee and for the valuable discussions during the committee meetings.

Thanks also to Prof. Jürgen Wienands, Prof. Christine Stadelmann-Nessler and Prof. Martin Weber for being part of my examination board.

Thanks to Prof. Rolf Daniel and Dr. Jacqueline Hollensteiner for the collaboration in the lung and gut microbiota project.

Thanks to Dr. Fred Lühder and Dr. Dmitri Lodygin for suggestions and ideas regarding my project.

Thanks to Roger Cugota Canals and Jana Schüttpelz for hours and hours of help during numerous extensive experiments.

Thanks to Michael Haberl for helping with the NGS analysis.

Thanks to Felicia Joy Flier, Adriane Stas and Simone Hamann for the technical assistance.

Thanks to Omar Diaz for IT support and for regularly fixing computer problems.

Thanks to Cathy Ludwig for proofreading my thesis.

Thanks to all my colleagues and friends at the IMSF for the great atmosphere and working environment.

Finally, I want to thank my parents Gundi and Günther, my brother Helge and my close friends for their support. 